\title{
Molecular mechanisms of myelin membrane biogenesis
}

\author{
Dissertation \\ Zur Erlangung des mathematisch-naturwissenschaftlichen Doktorgrades \\ "Doctor rerum naturalium" \\ der Georg-August-Universität Göttingen
}

vorgelegt von

Katarina Trajkovic

aus Pozarevac, Serbien

Göttingen, 2007 
Referent: Prof. Dr. Ernst Wimmer

Koreferent: Prof. Dr. Detlef Doenecke

Tag der mündlichen Prüfung: 05.07.2007 


\section{Baba Raisi}




\section{Table of contents}

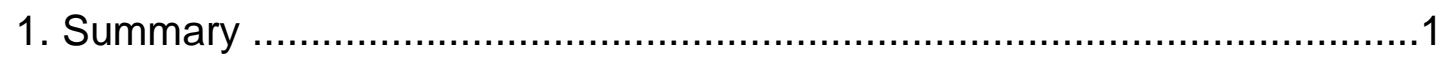

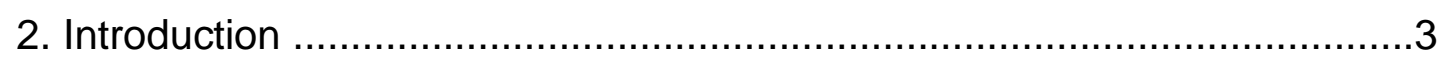

2.1. Biology of oligodendrocyte and myelin...................................... .3

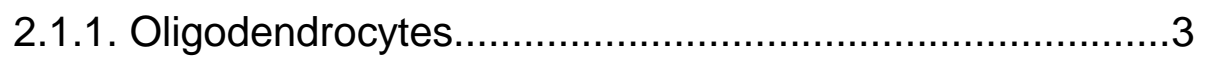

2.1.2. Structure of myelin ...................................................

2.1.3. The molecular composition of the myelin .......................

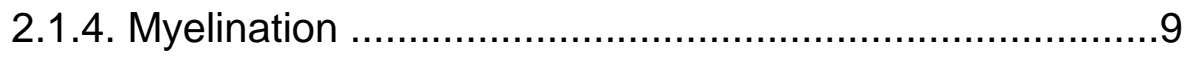

2.1.5. Sorting of myelin........................................................ 10

2.1.6. Function of myelin....................................................11

2.2. Membrane trafficking in the endosomal system..........................12

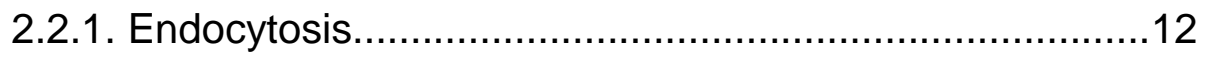

2.2.2. Early endosome and sorting into endosomal carrier vesicle/multivesicular body........................................17

2.2.3. Late endosome as a sorting station................................22

2.2.4. Communication of late stations of the endocytic pathway with the plasma membrane...............................23

2.2.5. Possible fates of intralumenal vesicles

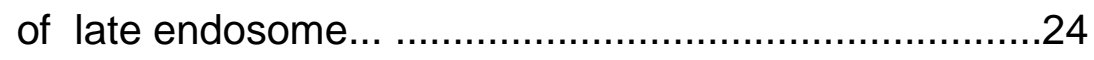

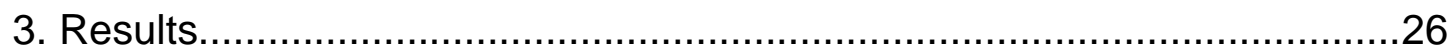

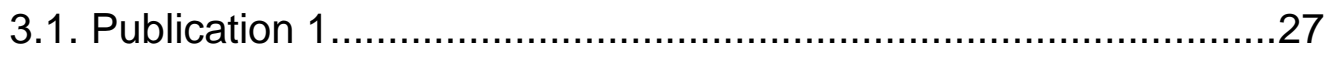

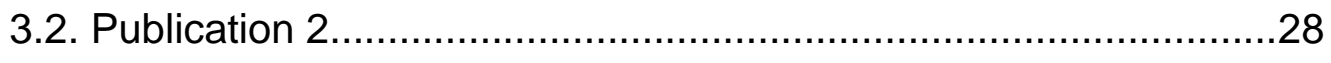

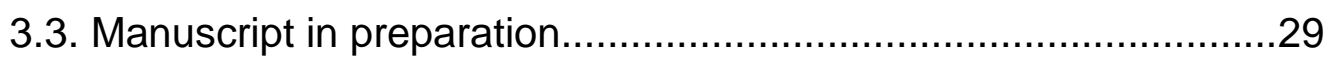

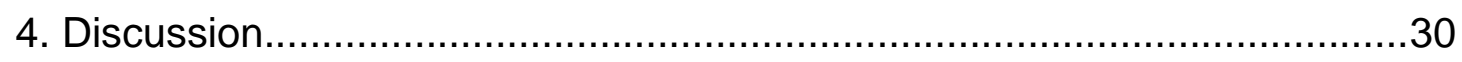

4.1. Regulation of endocytosis in oligodendrocytes..........................30

4.2. Sorting of proteolipid protein into multivesicular endosome...........32

4.3. Retrograde membrane transport of PLP....................................34

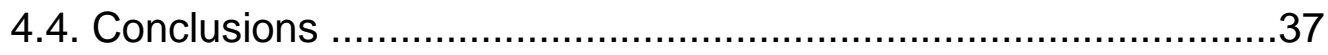

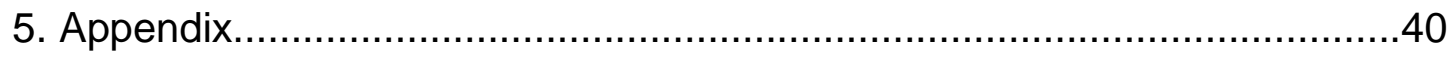

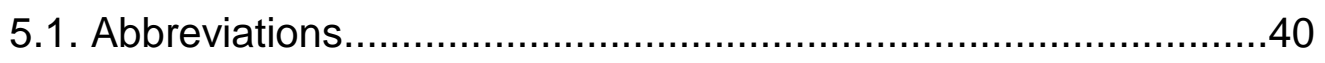

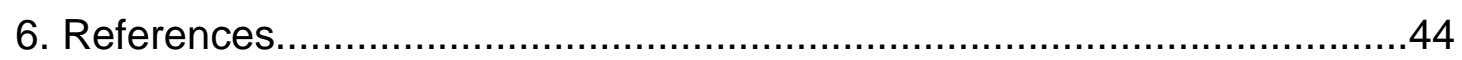


Acknowledgements.

Curriculum vitae.. 


\section{Summary}

In the vertebral nervous system, oligodendrocytes synthesize myelin membrane which enwrapes axons multiple times. Myelin insulates the axon from the extracellular environment and allows saltatory conduction of nerve impulses. Bidirectional interaction between neurons and oligodendrocytes is essential to coordinate myelin biogenesis. The aim of the present study was to investigate the membrane trafficking machinery which underlies myelination of the axonal tracts by oligodendrocytes, and the role of neuronal signals in the regulation of this process. For this purpose, we used neuron and oligodendrocyte cocultures as a cellular model.

We analyzed the distribution and trafficking of the major myelin membrane protein, the proteolipid protein (PLP), in oligodendrocytes cultured in the absence or presence of neurons. We observed that PLP is mainly localized in late endosomes (LE) in the cells cultured without neurons, whereas it is transferred to the plasma membrane in the presence of a soluble diffusible neuronal signal. The same redistribution was observed in situ, using mice brain sections. We found two reasons for the redistribution of PLP: reduced endocytosis and at the same time increased exocytosis of PLP from the endosomal storage pools in the presence of neuronal signals.

We showed that the endocytosis pathway used by PLP is clathrinindependent, but requires cholesterol, the function of dynamin and the actin cytoskeleton. In addition, this endocytosis pathway was regulated by the activity of the RhoA GTPase. Furthermore, we found that the transport of PLP into the lumen of endosomes was independent of the ESCRT-mediated pathway.

Using live microscopy, we showed that PLP-containing endosomes were transported to the cell periphery and were able to fuse with the plasma membrane. This pathway was regulated by the Rho-Src signaling system and controlled by neurons.

Our results reveal a mechanism of how neurons coordinate membrane trafficking in oligodendrocytes to control myelin membrane growth in the central nervous system. 


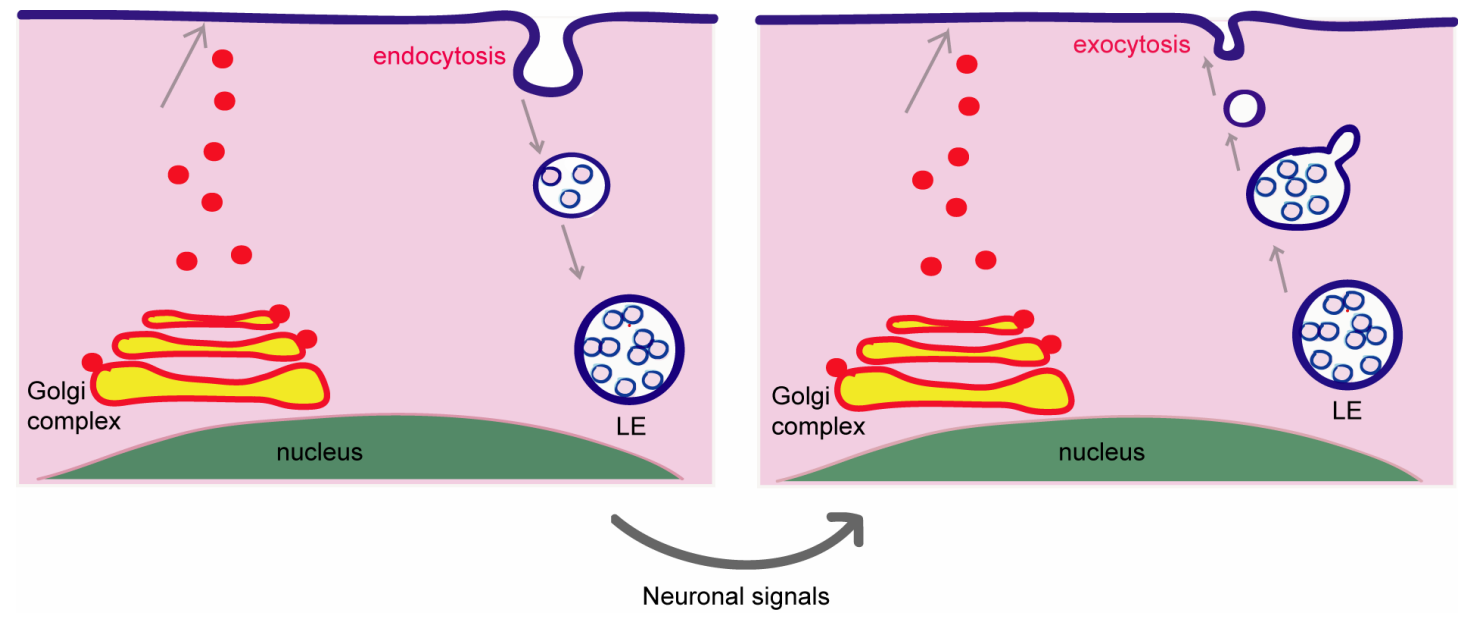

Figure 1. Neurons control the membrane trafficking machinery in oligodendrocytes. In the absence of neurons, PLP is internalized via a clathrin-independent endocytosis and stored in late endosomes (LEs). After receiving signals from neurons, the rate of endocytosis is reduced and transport from LE to the plasma membrane is triggered. Modified after Simons and Trajkovic, 2006. 


\section{Introduction}

\subsection{Biology of oligodendrocyte and myelin}

Multicellular animals require a highly sophisticated system to monitor, control and respond to the changes in their internal and external environment. The nervous system is one of the major coordinators of these functions. It includes the central (CNS) and the peripheral (PNS) nervous system. The vertebral nervous system is an extraordinarily complex structure composed of several cell types, which can be roughly divided into two groups: neurons and glia. Neurons are electrically excitable cells that transmit and process information. Glial cells constitute the large majority of the cells in the nervous system, and they are represented by three major types of cells: astrocytes, microglia and oligodendrocytes in the CNS, and the Schwann cells in the PNS. These cells participate actively in the physiological functioning of the brain, and their dysfunction leads to various neurological diseases.

\subsubsection{Oligodendrocytes}

Oligodendrocytes form myelin - an insulating multi-lamellar sheath wrapped in a spiral around axons. Mature oligodendrocytes possess a large number of processes. Each of them connects distinct myelinated segments of many different axons (Bunge et al., 1962; Bunge, 1968). There is some morphological heterogeneity among these cells, including the number of processes that form myelin sheaths, which varies from 1 to 40 (Baumann and Pham-Dinh, 2001). All of these cells go through a series of stages during their development, defined by different morphological features and molecular markers (Pfeiffer et al., 1993). Some of these markers will be described in more detail in the section on myelin. Together with the morphological criteria, these markers facilitate the identification of distinct developmental stages of oligodendrocytes. 
In the mammalian brain, both neurons and glia arise from the proliferating neuroepithelial cells of the ventricular and subventricular zone (Doetsch et al., 1997). The subventricular zone is a mosaic of multipotential precursors (Levison and Goldman, 1993) where environmental cues influence the fate of these cells (Jensen and Raff, 1997). Oligodendrocyte precursor cells (OPCs) arise from the subventricular zone at very early stages during embryonic life. They are monopolar cells expressing the plateledderived growth factor $\alpha$-receptor (PDGFR- $\alpha$ ) (Pringle and Richardson, 1993), but also myelin proteins CNP (2',3'-Cyclic nucleotide-3'-phosphohydrolase) (Yu et al., 1994) and DM20, the shorter splice-isoform of proteolipid protein (PLP) (Timsit et al., 1992). The next stage in the oligodendrocyte lineage are the proliferating oligodendrocyte progenitor cells, which migrate from the place of their origin into the developing white matter throughout the brain (Frost et al., 1996; Levison and Goldman, 1993). These cells have been characterized by their bipolar morphology and the presence of specific markers, e.g. glycolipid GD3 (Hardy and Reynolds, 1991) and proteoglycan chondroitin sulfate (NG2) (Nishiyama et al., 1996). After oligodendrocyte progenitor cells have reached their final target, they transform into preoligodendrocytes, multiprocessed cells which keep the property of cell division and which are recognized by the antibody $\mathrm{O} 4$. This antibody reacts with sulfatides and as yet unidentified glycolipids (Bansal et al., 1989). The preoligodendrocytes become immature oligodendrocytes characterized by the appearance of the lipid determinant galactosylceramide (GalC) and the loss of expression of GD3 on the cell surface. These cells exit the cell cycle and start the sequential expression of the mature oligodendrocyte-markers, myelin basic protein (MBP), myelin-associated glycoprotein (MAG) and PLP (DuboisDalcq et al., 1986; Hardy and Reynolds, 1991; Monge et al., 1986; Pfeiffer et al., 1993). The presence of myelin/oligodendrocyte glycoprotein (MOG) corresponds to the late stages of maturation (Solly et al., 1996). Mature oligodendrocytes can be differentiated further into premyelinating oligodendrocytes and myelin-forming oligodendrocytes, and this transition requires the presence of neurons (Baumann and Pham-Dinh, 2001).

However, the differentiation of oligodendrocytes in cell culture occurs normally in the absence of neurons (Dubois-Dalcq et al., 1986; Mirsky et al., 
1980). Therefore it seems that they have an intrinsic program defining the end of proliferation and their characteristic sequence of developmental steps (Temple and Raff, 1986), but this program is modulated by external factors coming from the surrounding cells. Neurons control the development of oligodendrocytes by regulating their proliferation, differentiation and survival (Barres and Raff, 1999). The signals are important to match the number of oligodendrocytes to the axonal surface requiring myelination. Several growth and trophic factors secreted by both neurons and astrocytes, such as PDGFA, FGF-2, IGF-1, NT-3 and CNTF, have been shown to regulate oligodendrocyte development (Baron et al., 2005; Barres and Raff, 1994; Miller, 2002). Neuregulins (NRGs), produced by neurons, stimulate terminal differentiation (Park et al., 2001) and the survival of maturing oligodendrocytes (Carteron et al., 2006; Fernandez et al., 2000; Flores et al., 2000). However, they also induce proliferation (Canoll et al., 1996) and inhibit maturation in OPC cultures (Canoll et al., 1999). Another example of an axonally derived signaling system is the Notch pathway. OPCs express the Notch 1 receptor, whose interaction with Jagged 1 located at the axonal surface results in inhibition of the differentiation of oligodendrocytes (Wang et al., 1998). The expression of the neuronal Jagged 1 is developmentally regulated, decreasing at the time when myelination starts, which suggests that this signaling system plays a role in the timing of myelination.

\subsubsection{Structure of myelin}

The major task of oligodendrocytes and Schwann cells is the production of myelin, a function no other cell type is able to carry out. Myelin is a spiral structure generated from the extensions of the plasma membrane of the oligodendrocytes in the CNS or the Schwann cells in the PNS. The myelin sheath around most axons is the hallmark of the vertebrate nervous system and represents its most abundant membrane. Myelin has a unique, segmental structure (Figure 2.1). The spiral wrapping of oligodendrocyte membrane extensions results in the periodic appearance of the myelin sheath segment with alternating concentric electron-dense and light layers (Baumann 
and Pham-Dinh, 2001), named the major dense line and the intraperiod line, respectively. The major dense line is formed by the cytoplasmic surfaces of the myelinating membrane which are brought into close apposition, whereas the fused two outer leaflets of this membrane represent the intraperiod line.
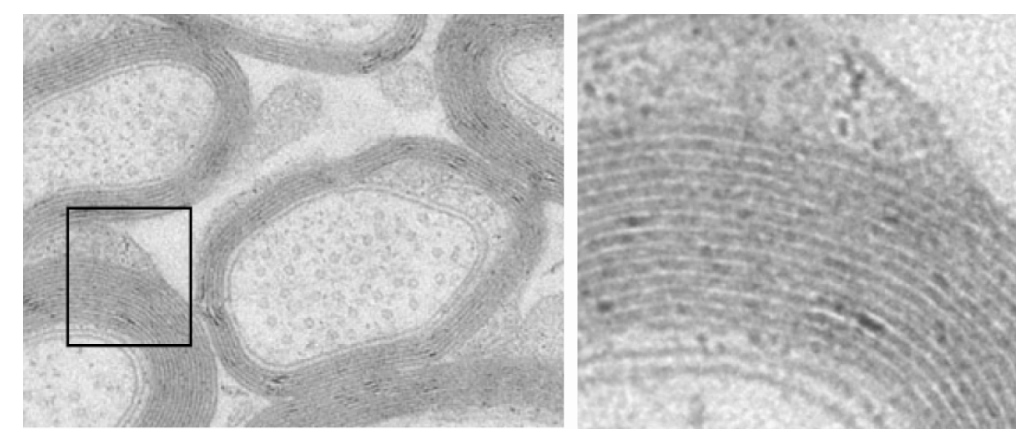

Figure 2.1. Myelinated axon: An electron micrograph of myelinated nerve fibers within the optic nerve. Modified after Simons and Trajkovic (2006).

Myelin also displays a periodic structure on the longitudinal sections, with regularly recurring 150-200 $\mu \mathrm{m}$ long myelinated segments or internodes (Butt and Ransom, 1989), which are separated by spaces where myelin is lacking, the nodes of Ranvier (Bunge, 1968). From most of the myelin, large parts of the cytoplasm is extruded leading to the characteristic appearance of compact myelin. However, some parts of the myelin are excluded from the compaction process. Schmidt-Lanterman incisures correspond to the cytoplasmic surfaces that have not formed the major dense line, thereby creating the uncompacted clefts filled with the remnant cytoplasm. These clefts extend across the whole sheath, thereby making the cytoplasm on the outside of the sheath confluent with that of the inside part. The expanded cytoplasm-containing lateral loops, which flank the node of Ranvier from either side, represent the next uncompacted element of the myelin. These loops form the paranodal region or paranode. Paranodal loops establish septate-like junctions with the axonal membrane.

Myelinated axons are organized into paranodal and juxtaparanodal longitudinal regions centered around the node of Ranvier (Figure 2.2). These three domains - node, paranode and juxtaparanode - exhibit the complex structure, and importantly, the differential distribution of ion channels. At the 
nodes of Ranvier, the axonal membrane accumulates voltage-gated sodium channels. The adjacent paranodal domain with its junctions inhibits the lateral diffusion of proteins between the node of Ranvier and the juxtaparanode. The juxtaparanodal domain lies just under the compact myelin sheath next to the paranodes, and includes two shaker-type potassium channels, $\mathrm{K}_{\mathrm{v}} 1.1$ and $\mathrm{K}_{\mathrm{v}}$ 1.2. The concentration of the sodium channels restricts the generation of the action potentials to the nodes of Ranvier. Signals from oligodendrocytes to neurons are necessary to cluster multiprotein complexes in the axonal membrane into distinct subdomains at the nodes of Ranvier (Pedraza et al., 2001; Poliak and Peles, 2003; Salzer, 2003; Scherer and Arroyo, 2002).

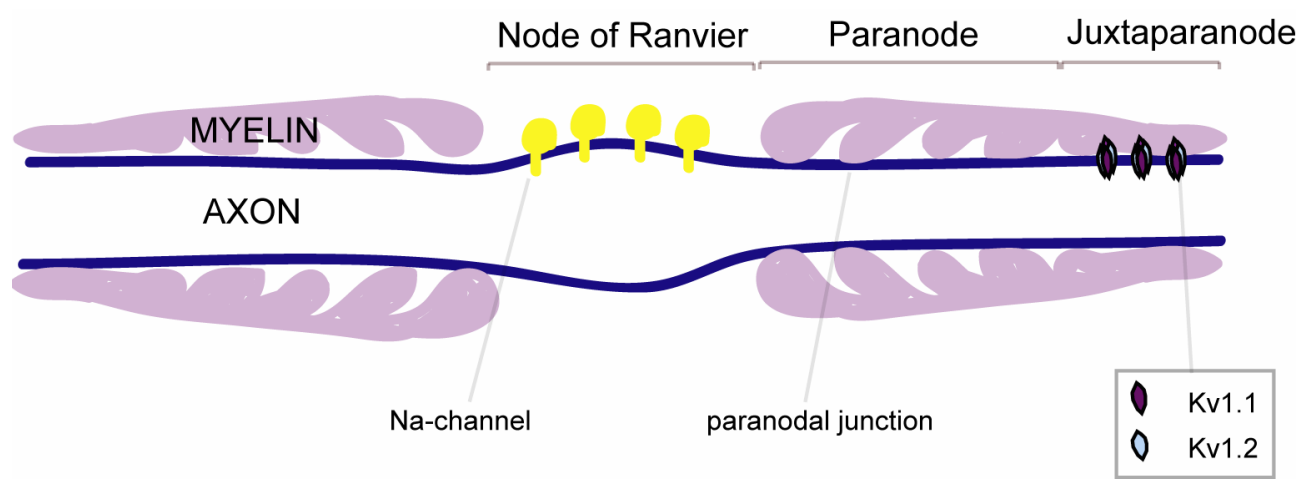

Figure 2.2. Schematic overview of domains at the node of Ranvier. Nodes accumulate voltage-gated sodium channels. Juxtaparanodes include shaker-type potassium channels $K_{v} 1.1$ and $K_{v} 1$.2. Paranodal loops of myelin establish septate-like junctions with the axonal membrane in the paranode. Modified after Simons and Trajkovic (2006).

\subsubsection{The molecular composition of myelin}

Apart from the unique morphology, the molecular composition is another peculiarity of the myelin membrane. In contrast to most plasma membranes, myelin is a poorly hydrated, lipid-rich membrane (lipids constitute $70 \%$ of the dry myelin weight) with the high content of glycosphingolipids and cholesterol. The major glycosphingolipids in myelin are galactosylceramide and its sulfated derivative sulfatide ( $20 \%$ of lipid dry weight). There is also an unusually high proportion of ethanolamine phosphoglycerides in the plasmalogen form, which accounts for one-third of the phospholipids. This 
specific lipid structure is responsible for the insulating properties of the myelin sheath.

Myelin contains a relatively simple array of proteins, distributed unequally in distinct domains. Myelin basic protein (MBP), and the proteolipid proteins (PLP and its shorter splice-isoform DM20) are the two major CNS myelin proteins, and PO the major PNS myelin protein. These proteins are predominantly found in the compact, internodal myelin.

MBP constitutes as much as $30 \%$ of the myelin protein content. In fact, it is a family of isoforms with different molecular masses, coded by alternative transcripts from the single MBP gene. It is a peripheral membrane protein localized to the major dense line. The mechanistic function of MBP in the formation of myelin has not yet been fully understood. The binding of the positively charged MBP to the negatively charged inner leaflet of the plasma membrane suggests that it can function as a lipid coupler by bringing the layers of myelin close together. Recent data indicate that neurons increase the lipid packing of the myelin-forming bilayer in oligodendrocytes and that MBP is involved in this process of plasma membrane rearrangement (Fitzner et al., 2006). Direct evidence that MBPs play a major role in myelin compaction came from the studies of the shiverer mouse, a severely hypomyelinated mutant carrying a large deletion of the MBP gene (Roach et al., 1985).

PLP, the most abundant myelin protein in the mammalian CNS, is a highly hydrophobic molecule, 276 amino acids long, with molecular weight of $26 \mathrm{kDa}$. The hydrophobicity is further increased by the esterification with long fatty acids (Weimbs and Stoffel, 1992). It is an integral membrane protein which traverses the plasma membrane bilayer four times with its transmembrane $\alpha$-helices. In addition to the transmembrane domains, PLP comprises two extracytoplasmic and three intracytoplasmic domains (including the $\mathrm{NH}_{2}$ and $\mathrm{COOH}$ termini). The major part of this protein localizes to the intraperiod line (Weimbs and Stoffel, 1992). The jimpy and other mutations in the PLP gene lead to severe dysmyelinating phenotypes, in contrast to the total depletion of the PLP/DM20 (Coetzee et al., 1999). In the PLP null mutant mice, oligodendrocytes are still able to myelinate axons and assemble compacted myelin sheaths, although the structure of myelin is 
impaired and late-onset neurodegeneration is observed (Griffiths et al., 1998; Klugmann et al., 1997).

Some of the quantitatively minor myelin proteins of the CNS are: MAG, myelin oligodendrocyte protein, claudin11/oligodendrocyte-specific protein (OSP), neurofascin-155, the enzyme 2',3'-Cyclic nucleotide-3'phosphohydrolase (CNP) and myelin/oligodendrocyte glycoprotein (MOG).

\subsubsection{Myelination}

Myelination of the axonal tracts by oligodendrocytes primarily takes place in early postnatal life. This process is divided into several steps: 1) the recognition of and adhesion of oligodendrocytes to the appropriate axon; 2) the synthesis and transport of myelin components to the axon; 3) the wrapping of the myelin membrane around axons and 4) the compaction of the myelin sheath. The greatest part of this process takes places within a very short period of time - during the active phase of myelination, in which each oligodendrocyte must produce as much as $\sim 5-50 \times 10^{3} \mu \mathrm{m}^{2}$ of myelin membrane surface area per day (Pfeiffer et al., 1993). Oligodendrocytes must have developed specific mechanisms to be able to coordinate intracellular sorting and transport at such a high production rate.

Myelination is carefully controlled by external factors. The timing of myelination is crucial because the ensheathment of axons must not occur before the neurons signal to oligodendrocytes. The morphological features and activity of neurons have an important role: oligodendrocytes do not wrap their plasma membrane randomly around neuronal processes but carefully select axons that have diameter of $>0.2 \mu \mathrm{m}$; they also exclude dendrites. Neuronal electrical activity leads to the secretion of promyelinating factors, such as adenosine, from neurons (Stevens et al., 2002). Conversely, impulses in premyelinating axons in the PNS trigger the release of adenosine triphosphate, which inhibits the proliferation and the differentiation of Schwann cells (Stevens and Fields, 2000). The electrical activity of neurons also leads to a change in the expression pattern of axonal proteins (Coman et al., 2005). The removal of some of these proteins, such as the polysialated adhesion 
molecule NCAM, is essential and allows the myelination process to proceed (Charles et al., 2000). Recent work reveals the role of astrocytes in the initiation of myelination in response to electrical impulses: they appear to release a leukemia inhibitory factor (LIF), which then stimulates oligodendrocytes (Ishibashi et al., 2006).

\subsubsection{Sorting of myelin}

Myelin assembly and trafficking is much more than the expression of myelin genes and involves the assembly of particular components in a temporally and spatially regulated manner. This requires specific sorting and transport mechanisms for the delivery of myelin membrane components from their source to the newly forming myelin membrane (Kramer et al., 1997; Pfeiffer et al., 1993). Individual myelin components are synthesized in oligodendrocytes at several subcellular localizations and transported by different mechanisms to the growing myelin membrane.

PLP and DM20 are transported by vesicular transport through the biosynthetic pathway to myelin (Colman et al., 1982). On its way to the plasma membrane, PLP/DM20 associates with cholesterol and galactosylceramide in the Golgi complex, which might assist the targeting of PLP/DM20 to myelin (Simons et al., 2000). The first hint of the signal determining the selective targeting of PLP/DM20 to myelin came from work carried out on transgenic mice showing that the $\mathrm{N}$-terminal 13 amino acids of PLP are sufficient to target a cytoplasmic fusion protein (lacZ) to the myelin membrane (Wight et al., 1993). Recent work indicates that the palmitoylation of this sequence is necessary for the selective sorting of myelin (Schneider et al., 2005). These studies, amongst others, suggest that lipid rafts are involved in the transport of proteins to myelin (Kim et al., 1995; Kramer et al., 1997; Marta et al., 2003; Simons et al., 2002; Taylor et al., 2002).

In contrast to PLP, the targeting of MBP to myelin depends on the transport of the respective MBP mRNAs. The MBP mRNA is assembled into granules in the perykarion of oligodendrocytes, transported along processes and localized at the myelin membrane (Ainger et al., 1993). The transport of 
the RNA to the plasma membrane depends on a 21-nucleotide sequence, the RNA transport signal, in the 3'UTR of the MBP mRNA (Ainger et al., 1997). It is not known whether the transport and/or final localization of the mRNA or the protein are regulated by extrinsic signals. However, the finding that MBP is initially synthesized in the cell body and localized within processes and the myelin sheath later during the development suggests that its transport may be under developmental control (Shiota et al., 1989).

\subsubsection{Function of myelin}

The insulating properties of myelin together with its organization in repeating segments along the axons are a prerequisite for the fast, saltatory conduction of impulses in the nerve fibers in the vertebrate nervous system. The nerve impulse generated at the node of Ranvier jumps from node to node due to the low capacitance and the high resistance of the myelin sheath. The major advantages conferred to the vertebrate nervous system by the myelin sheath are high-velocity conduction, fidelity of long-distance signaling and spatial economy, in contrast to the invertebrate nervous system, in which rapid conduction is achieved by the increased diameter of the axons. In addition, myelin plays an important role in axonal development and maintenance (Lappe-Siefke et al., 2003), but also inhibits the regeneration of the injured axons within the adult mammalian CNS. The essential role of myelin in the functioning of the nervous system is highlighted by severe neurological symptoms that accompany different demyelinating diseases such as multiple sclerosis. 


\subsection{Membrane trafficking in the endosomal system}

\subsubsection{Endocytosis}

Endocytosis is the process by which cells take up plasma membrane components, extracellular ligands and soluble molecules. It has an essential role in many cellular processes, like nutrient uptake, the recycling of synaptic vesicles, regulation of the surface expression of receptors, remodeling of the plasma membrane and the generation of cell polarity. The best described endocytosis pathway is clathrin-mediated endocytosis. This form of endocytosis begins at clathrin-coated pits, the specialized regions which occupy about $2 \%$ of the total plasma membrane surface. Clathrin-coated pits invaginate into the cell and pinch off to form clathrin-coated vesicles. The assembly of the clathrin coat is mediated by several cytosolic proteins, including clathrin adaptor AP-2, eps15 (substrate for the tyrosine kinase of the epidermal growth factor receptor) and epsin (Maldonado-Baez and Wendland, 2006). Small guanosine triphosphatase (GTPase) dynamin together with other cytosolic proteins regulates the fission of the vesicles from the plasma membrane. Once the vesicle is released, the clathrin coat is rapidly disassembled, and the vesicle can fuse with an early endosome.

Recently, a number of clathrin-independent internalization pathways have been identified. These pathways share some of the machinery among each other and with the clathrin-mediated pathway, but they all have certain specificities which identify them as separate pathways (Kirkham and Parton, 2005) (Figure 2.3). Although the classification of these pathways is still under debate, the majority of the cargo entering the cell in a clathrin-independent manner utilizes macropinocytosis, caveolae - dependent or a clathrinindependent and caveolae-independent pathways either requiring dynamin or not.

Macropinocytosis is the internalization of significant areas of the plasma membrane together with huge amounts of extracellular fluid, followed by the formation of large $(>1 \mu \mathrm{m})$ irregular vesicles called macropinosomes. This process is coupled with membrane ruffling coordinated through the 
activity of the small GTPase Rac1. The small GTPases ARF6 (ADPribosylation factor 6) (Radhakrishna et al., 1996), and cdc42 (Garrett et al., 2000; Symons and Rusk, 2003) have been implicated in macropinocytosis.

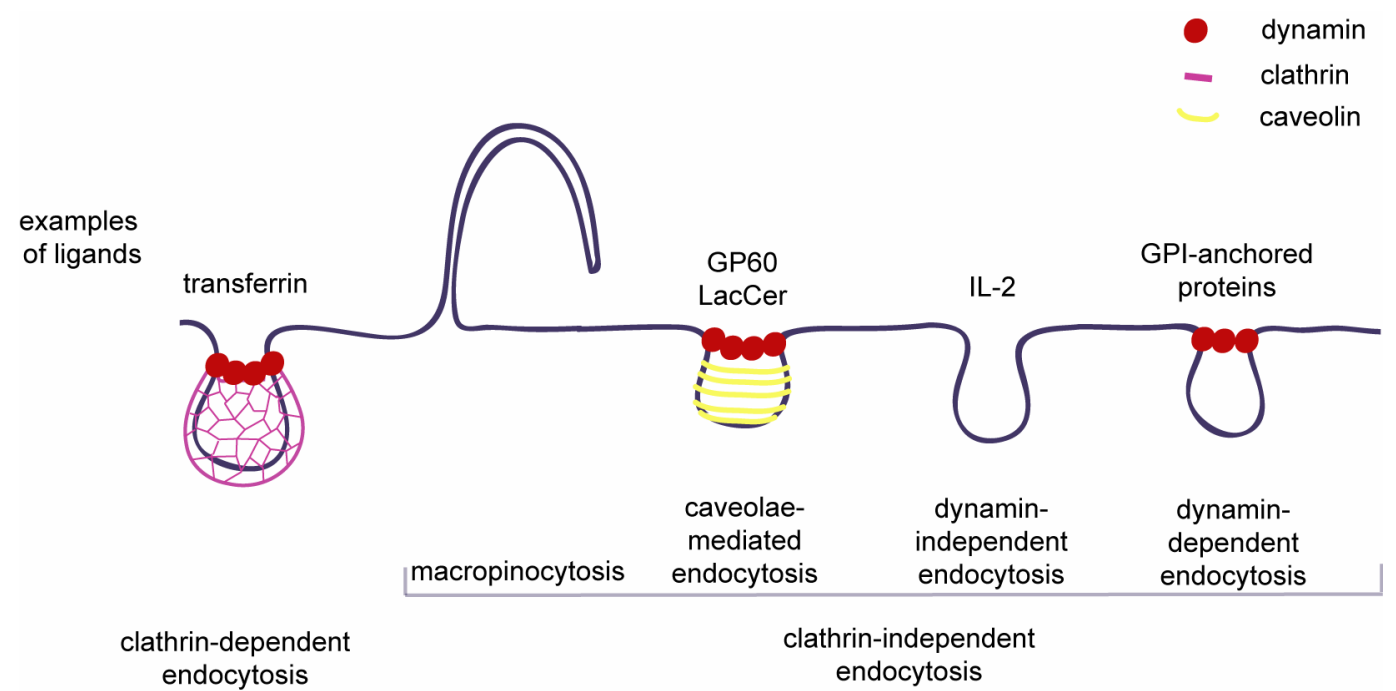

Figure 2.3. The visualization of different endocytic pathways. Abbreviations: LacCer, lactosylceramide; IL-2, interleukin-2; GPI, glycosylphosphatidylinositol. Modified after Kirkham and Parton (2005).

Caveolae are flask-shaped, non-coated invaginations of the plasma membrane present in most cell types (Kurzchalia and Parton, 1999). Their key component is caveolin, a cholesterol-binding protein that forms high-ordered oligomers and sequesters lipid-raft components. Caveolin-1 knockout mice lack morphologically distinguishable caveolae, which suggests that the expression of caveolins is absolutely required for the formation of caveolae. The budding of caveolae is mediated by dynamin (Henley et al., 1998; Oh et al., 1998) and is regulated by a large number of kinases (Pelkmans, 2005). Furthermore, rearrangements of actin cytoskeleton is required for endocytosis of caveolae (Pelkmans et al., 2002). Some specific markers of the caveolaemediated pathway are the albumin-binding glycoprotein GP60, Simian Virus 40 (SV40) and lactosylceramide (LacCer) (Kirkham and Parton, 2005).

One interesting difference between caveolae and clathrin-mediated endocytosis is the stable association of the caveolin-containing coat of the budding vesicles with the plasma membrane (Pelkmans et al., 2004), as opposed to a clathrin coat, which is rapidly removed after vesicle detaches from the plasma membrane (Pelkmans, 2005; Tagawa et al., 2005). In fact, 
the clathrin-coated vesicles lose their identity at the time when they fuse with early endosomes, whereas caveolae can either transiently fuse with endosomes following a kiss-and-run mode of interaction with a completely retained identity, or become stably associated with a relatively stationary, large, multi-caveolar assembly with neutral $\mathrm{pH}$ - the caveosome (Pelkmans, 2005). The caveosome is distinct from the transferrin-labeled, acidic early endosome. This organelle is permanently present in the cell independent of the activity of caveolae-mediated endocytosis, and it has an ability to further interact and exchange material with different compartments in a kiss-and-run manner (Pelkmans, 2005).

Another clathrin-independent pathway distinct from caveolae-mediated endocytosis has been described (Sabharanjak et al., 2002). Using GPIanchored proteins as marker proteins, a novel clathrin- and dynaminindependent endocytic pathway sensitive to cholesterol depletion was identified. The initial compartment where GPI-anchored proteins seem to enter appears to be a network of tubular carriers named GPI-enriched endosomal compartment (GEEC). This form of endocytosis is regulated by the small GTPase cdc42, but not by other Rho family members. Other studies have implicated a role of the GTP-binding protein ARF6, as well as phosphatidylinositol $(4,5)$ bisphosphate $\left(\operatorname{Ptdlns}(4,5) \mathrm{P}_{2}\right)$ in this process (Naslavsky et al,2004). Recent data indicate that the endocytosis of GPIanchored proteins may require the function of flotillin-1 (Glebov et al., 2006).

A somewhat related endocytosis pathway is used by the $\beta$ subunit of interleukin-2 (IL-2) receptor (Lamaze et al., 2001). This pathway is sensitive to cholesterol depletion and clathrin-independent, but it requires the activity of dynamin and RhoA GTPase. A similar clathrin - independent, dynamin dependent endocytosis is followed by the yc cytokine receptor (Sauvonnet et al., 2005).

In contrast to caveolae-mediated endocytosis, the pathways used by both the IL-2 receptor (Lamaze et al., 2001) and GPI-anchored proteins (Sabharanjak et al., 2002) converge with the clathrin-mediated endocytosis system within the common endosomal compartment (Hemar et al., 1995; Sabharanjak et al., 2002). 
The caveolae-mediated endocytosis system seems to be special in a sense that it segregates its cargo from the endosomal systems used by most other cargos. This feature is mediated by caveolin, which acts as a scaffold keeping its cargo concentrated not only during the initial phase of internalization, but also during intra-endosomal transport. It is possible that caveolae-mediated endocytosis is a more specialized form of the clathrinindependent and caveolae-independent endocytosis pathways. Their close relation is clearly shown by the fact that depletion of caveolae results in the uptake of caveolar cargo by these clathrin-independent and caveolaeindependent pathways (Damm et al., 2005).

The borders between clathrin-dependent and -independent pathways are also blurred to some extent. For example, the antrax and tetanus toxins use a cholesterol depletion-sensitive, but clathrin-dependent pathway for internalization (Abrami et al., 2003; Deinhardt et al., 2006). It seems that these toxins require lipid-rafts in the initial phase for the concentration of cargo, but they subsequently dissociate from lipid-rafts and enter clathrincoated pits. There are also examples of cargo that can use either one or another pathway depending on specific signals. Under low concentration of EGF, the EGF receptor enters clathrin-coated pits, whereas high concentrations of EGF trigger the internalization via a clathrin-independent pathway (Sigismund et al., 2005). The specific determinant seems to be the monoubiquitination of the receptor induced by high concentration of EGF, which acts as a signal for the recruitment of epsin and the subsequent endocytosis by a clathrin-independent pathway. Another interesting example for differential sorting is the TGF- $\beta$ receptor: the uptake via caveolae leads to its degradation, while clathrin-dependent uptake induces the generation of the receptor-mediated signal (Di Guglielmo et al., 2003). There are also examples of how different pathways can lead to the transport to the same terminal station. The targeting of cholera toxin to Golgi complex and subsequent acquiring toxicity is possible by entry through clathrin-dependent and independent pathways (Orlandi and Fishman, 1998). Likewise, sphingomyelin can reach the Golgi complex through both clathrin-dependent or -independent pathways (Puri et al., 2001).There are also examples of differential uptake mechanisms dependent on the cell type: the cellular isoform of the prion 
protein $\mathrm{PrP}^{\mathrm{c}}$ is a raft-associated protein internalized via clathrin-mediated endocytosis in neurons (Sunyach et al., 2003), or via caveolae in nonneuronal cells (Peters et al., 2003).

Endocytosed molecules can undergo different fates, some being directed towards lysosomes for degradation and others being retrieved for reutilization. The decision regarding their fate can be taken during any of the following steps: on the level of the plasma membrane (as discussed in the section above), in early or in late endosomes (van der Goot and Gruenberg, 2006). Schematic overview of the endosomal trafficking is presented in the Figure 2.4.

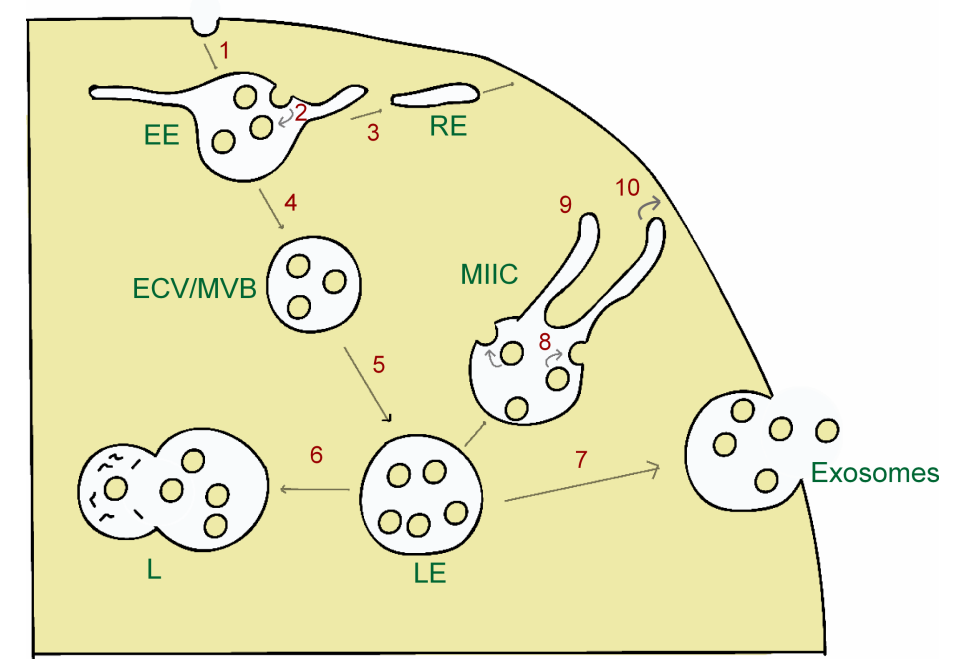

Figure 2.4. Overview of endosomal trafficking. Endocytosed cargo is targeted to early endosome (1), from where it can be recycled back to the plasma membrane via recycling endosome (2), or sorted into intralumenal vesicles (3) of an endosomal carrier vesicle/multivesicular body (ECV/MVB) (4). ECV/MVBs deliver their content to the late endosome (5). Late endosome can fuse with the lysosome thereby leading to the degradation of cargo (6). Alternatively, it can fuse with the plasma membrane and release its intralumenal vesicles which then become exosomes (7). Third possibility is back-fusion of intralumenal vesicles with the endosomal perimeter membrane (8), followed by the formation of tubular extensions (9), which eventually fuse with the plasma membrane (10), as in the case of MHC-II compartment in dendritic cells. Abbreviations: EE, early endosome; RE, recycling endosome; ECV/MVB, endosomal carrier vesicle/multivesicular body; LE, late endosome; L, lysosome; MIIC, MHC class II compartment; 


\subsubsection{Early endosome and sorting into endosomal carrier vesicle/multivesicular body}

The early endosome is a dynamic compartment (Gruenberg et al., 1989), which acts as a sorting station for the internalized cargo downstream of the plasma membrane. There is evidence for the existence of different early endosomal populations reached by two unequal types of clathrin-coated vesicles (Lakadamyali et al., 2006). The first type fuses with the so-called dynamic early endosome and its content is targeted further to a lysosome, whereas the content of the second type recycles to the cell surface via static early endosomes. The presence of distinct membrane domains within a single early endosome has also been shown (Gruenberg, 2001). These domains seem to be involved in sorting (Gruenberg, 2001). Once in early endosomes, housekeeping receptors can recycle back to the surface directly or through the recycling endosome, whereas some other molecules follow a transport pathway to the Golgi complex or endoplasmic reticulum (ER) (Maxfield and McGraw, 2004; Perret et al., 2005). Alternatively, material can be delivered to late endosomes and lysosomes. There are currently two hypotheses regarding trafficking between the early and late endosome: the maturation model, in which early and late endosomes reflect different stages of the same compartment, and the stable-compartment model, proposing two stable compartments with intermediate carrier that shuttles material among them. The maturation model proposes the gradual replacement of the early endosomal marker Rab5 with the late endosomal marker Rab7, as shown recently by live-cell imaging (Rink et al., 2005). Conversely, another independent study shows that Rab7-enriched domains form on the Rab5positive early endosome and subsequently bud away (Vonderheit and Helenius, 2005). Both models agree with the existence of endosomal carrier vesicle/multivesicular body (ECV/MVB), the intermediate which contains endocytosed tracers after segregation from recycling receptors, but before reaching lysosomes (Geuze et al., 1983; Miller et al., 1986; Mueller and Hubbard, 1986). ECV/MVBs display the characteristic accumulation of intralumenal vesicles (ILVs) and they can fuse with - or mature into - late endosomes, which eventually fuse with lysosomes (Bright et al., 2005). 
The formation of ECV/MVBs requires the sorting of the proteins into vesicles that invaginate into the interior of the early endosome (Gruenberg and Stenmark, 2004). The collecting of the proteins within ILVs requires four ESCRTs (Endosomal Sorting Complexes Required for Transport): ESCRT-0, -I, -II and -III, which act sequentially in the sorting process (Babst, 2005; Babst et al., 2002a; Babst et al., 2002b; Katzmann et al., 2001). In yeast, ESCRT-0 is represented by the complex of Hse1 and Vps27 (vacuolar protein sorting 27), which binds the ubiqitinated cargo (Bilodeau et al., 2002). ESCRT-I, heterotrimer composed of Vps23, Vps28 and Vps37 subunits, has the ability to interact with ubiqitinated cargo via Vps23 subunit (Katzmann et al., 2001). ESCRT-II, heterotrimer with subunits Vps22, Vps25 and Vps36, acts downstream from ESCRT-I (Babst et al., 2002b; Katzmann et al., 2002). ESCRT-II can also bind ubiquitin via its subunit Vps36, which harbors a ubiquitin-binding Npl4 zinc finger (NZF) domain (Alam et al., 2004; Meyer et al., 2002). ESCRT-III is a heteromultimer represented by two functional subcomplexes: Vps20/Snf7 and Vps2/Vps24, and it is recruited to the endosomal membrane by ESCRT-II (Babst et al., 2002a). ESCRT-III does not have a ubiquitin-binding site, but it is necessary for the recruitment of other proteins which function downstream from the cargo recognition (Babst et al., 2002a). There are two models of ESCRT function: the 'ubiquitin hand-off model' entails the transfer of cargo first from ESCRT-0 to ESCRT-I, and subsequently from ESCRT-I to ESCRT-II, whereas the alternative 'clustering' hypothesis suggests that the formation of the 'complex of complexes' Vps27/ESCRT-I/ESCRT-II leads to cargo clustering by simultaneous binding (Hurley and Emr, 2006). In addition to these complexes, there are several other proteins involved in the sorting process. Vps4 - an AAA-type ATP-ase (Babst et al., 1997; Babst et al., 1998) catalyses the release of ESCRT complexes from the endosomal membrane (Babst et al., 2002a; Babst et al., 1998; Katzmann et al., 2001). Bro1 recruits Doa4 (Luhtala and Odorizzi, 2004), the enzyme involved in the deubiquitination of the cargo prior to sorting into intralumenal membranes (Amerik et al., 2000; Babst et al., 2002b). Because the proteins involved in sorting into ECV/MVBs are evolutionarily conserved, they have been identified independently in several different species, which resulted in somewhat confusing nomenclature (Slagsvold et 
al., 2006). For example, human orthologues of Vps27 and Vps23 are Hrs (hepatocyte growth factor receptor substrate) and Tsg101 (tumor susceptibility gene 101), respectively, whereas Vps5 and Vps17 from yeast are functionally equivalent to the proteins of the nexin family in humans (Carlton et al., 2005). Mammalian homologue of the yeast protein Bro1 is Alix (ALG-2 interacting protein X, also known as AIP1). The sorting described for the mammalian system takes place in the following sequence of events: the process is initiated when Hrs recruits clathrin to form an atypical coat (Raiborg et al., 2001). Hrs concentrates ubiquitinated proteins destined for the degradation on the perimeter membrane of the early endosome (Raiborg et al., 2002; Urbe et al., 2003), and interacts with Tsg101, thereby recruiting ESCRT-I (Bache et al., 2003; Katzmann et al., 2003; Lu et al., 2003). Alix has been shown to interact with ESCRT-I via Tsg101 and with ESCRT-III via Vps32 (Martin-Serrano et al., 2003; Strack et al., 2003; von Schwedler et al., 2003). The sorting of the cargo into ECV/MVB, but not the further transport, is highly dependent on phosphatidylinositol-3-phosphate (PI3P) signaling (Petiot et al., 2003). A model for protein sorting into ECV/MVB is presented in the Figure 2.5.

In addition to its sorting function, the ESCRT network also drives the formation, invagination and fission of vesicles, which bud into the lumen of ECV/MVEs. Sorting into ILVs can be uncoupled from the vesicle formation (Urbe et al., 2003). The membrane invagination and the budding of the vesicles in the direction of endosomal lumen is topologically opposed to endocytosis, and therefore requires machinery different from that involved in the internalization from the plasma membrane. The structural analysis of the sorting machinery reveals the resemblance of the ESCRT-III complex to SNARE (soluble $\mathrm{N}$-ethylmaleimide-sensitive factor attachment protein receptor) proteins, which mediate membrane docking and fusion. This suggests the possible role of the ESCRT-III complex in the membrane fusion of the neck regions of the invaginated endosomal membrane, and the subsequent scission of the newly formed vesicle (Raiborg et al., 2003). Apart from the ESCRT-mediated budding, there are other possibilities for ILV formation. It is known that the unconventional phospholipid 
lysobisphosphatidic acid (LBPA) is highly enriched in ILVs (Kobayashi et al., 1998). Matsuo et al. (2004) found that the high abundance of LBPA in artificial liposome at acidic internal $\mathrm{pH}$ enhances inward vesiculation, presumably because of its inverted cone shape, which promotes the invagination of the membrane. They have also shown that this process is selectively controlled by Alix. LBPA is not detected in yeast (Russell et al., 2006), which suggests that this might be a vesiculation mechanism of higher organisms. The inward vesiculation may also require annexins (Mayran et al., 2003; White et al., 2006). Additionally, the cargo itself can influence the formation of the ECV/MVBs: the stimulation of cells with EGF significantly increases both the number of ILVs per ECV/MVB, and the number of ECV/MVBs (White et al., 2006).

It seems that there are different subpopulations of ECV/MVBs: ILVs enriched in LBPA are found in distinct compartments from those enriched in cholesterol (Mobius et al., 2003) or EGF receptor (White et al., 2006). EGF stimulation increases the biogenesis of only the latter compartment, and this process depends on annexin 1 (White et al., 2006). The fact that there are more types of these vesicles points to the existence of different pathways of ILV biogenesis. Some data support the idea that there are variations of the ESCRT-mediated sorting mechanism. Yeast proteins Sna3p (Bilodeau et al., 2002) and Cvt17p (Epple et al., 2003) and human $\delta$-opioid receptor (Hislop et al., 2004) do not require direct ubiquitination and ubiquitin interacting motifs (UIMs) of ESCRT-I for sorting into ILVs. Some of them can access ILVs by associating with other ubiquitinated proteins (Hislop et al., 2004), or binding downstream ESCRT members (Geminard et al., 2004; Strack et al., 2003). The first and, so far, the only protein shown to follow a ubiquitin-independent, ESCRT-independent pathway en route to ILVs is the melanocyte protein Pmel17 (Theos et al., 2006).

Interestingly, the ECV/MVB formation machinery is usurped by many retroviruses, including human immunodeficiency virus (HIV), which use this cellular pathway for their budding and spreading of the infection (Morita and Sundquist, 2004). Thus, this pathway may be a potential target for treatment of different retrovirus - induced diseases. 

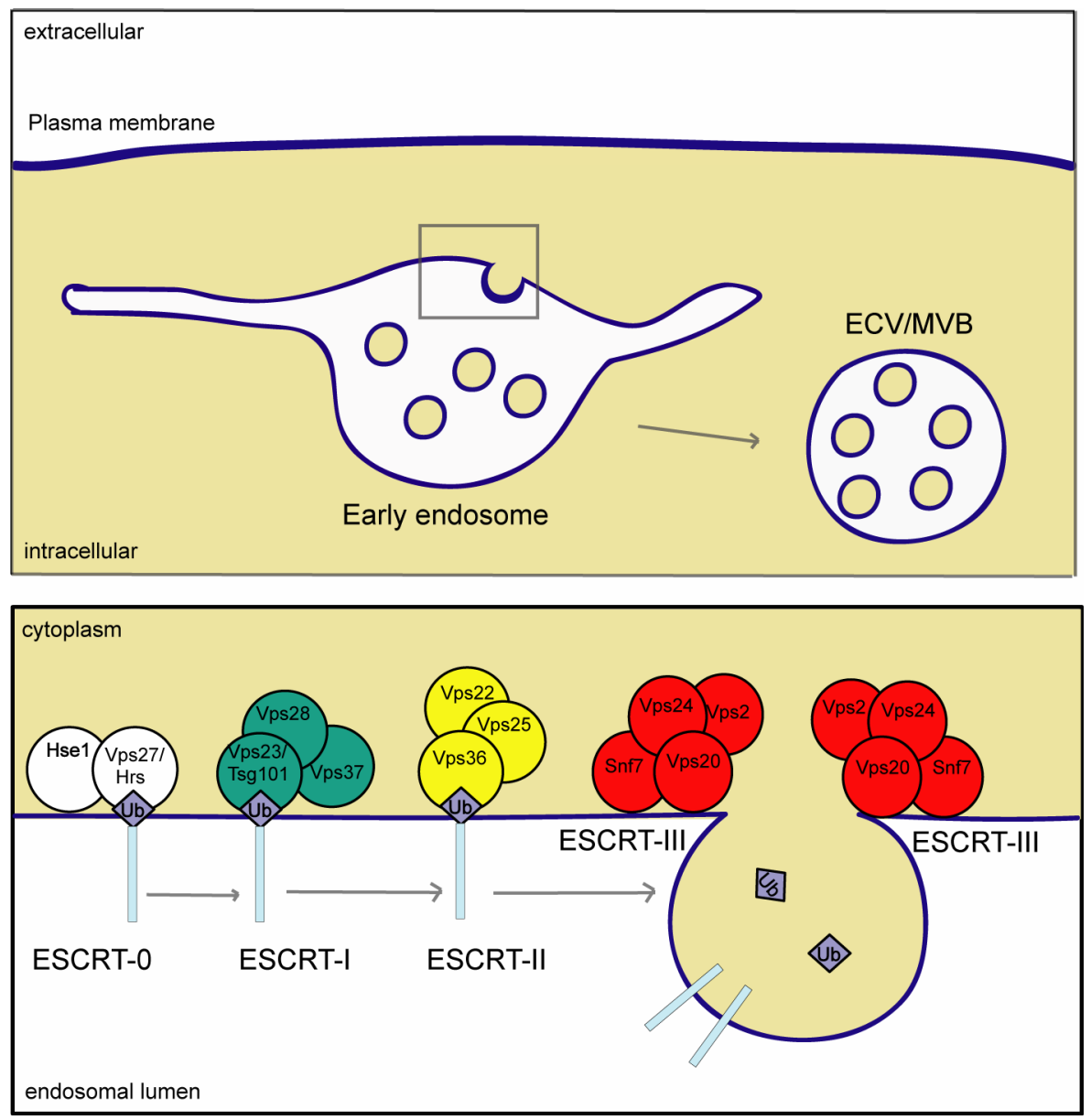

Figure 2.5. The organization of ESCRT-complexes and model for protein sorting into a multivesicular endosome. a) Schematic overview of the early endosome, which gives rise to an endosomal carrier vesicle/multivesicular body (ECV/MVB). b) Schematic representation of endosomal protein complexes required for transport (ESCRTs) and a model for sorting of ubiquitinated membrane proteins into ECV/MVB. Hrs subunit of ESCRT-0 recruits a ubiquitinated protein to the endosomal membrane. Subsequently, ubiquitinated cargo is delivered to ESCRT-I by interacting with Tsg101. The cargo is then relayed to ESCRT-II and transported into intralumenal vesicles, whose formation is mediated by ESCRT-III. Before vesicle scission, the protein is deubiquitinated. Abbreviations: Ub, ubiquitin. Modified after Raiborg et al. (2003). 


\subsubsection{Late endosome as a sorting station}

ECV/MVBs are translocated along microtubules towards the perinuclear area where they fuse with, or mature into late endosomes (Aniento et al., 1993; Bomsel et al., 1990; Parton et al., 1992). Late endosomes have a highly pleiomorphic structure with tubular, cisternal and multivesicular regions. They are highly acidic organelles $(\mathrm{pH} \sim 5.0-5.5)$, which contain biochemical markers, such as lysosomal-associated membrane protein-1 and -2 (Lamp-1 and Lamp-2), located on the limiting membrane of the organelle (Griffiths et al., 1988). Another resident of the limiting membrane is MLN64, a homologue of the mitochondrial steroidogenic acute regulatory protein (StAR) (Alpy et al., 2001). By contrast, CD63, a member of tetraspanin protein family, is found within intralumenal membranes (Escola et al., 1998), whereas mannose 6-phosphate receptor (M6PR) transits through these membranes en route to the Golgi complex (Griffiths et al., 1988). LBPA is another abundant component of the intralumenal membranes of late endosomes (Kobayashi et al., 1998). Recent data from Sobo et al. (2007) provide further insight into the structural organization of late endosomes. They identified two different raft-like domains: the first domain contained flotillin-1 in the limiting membrane, whereas the second was enriched in GPI-anchored proteins within the ILVs. Some studies indicate the existence of two lateendosomal subpopulations, one enriched in Rab7 and the other in Rab9 endosomal marker (Barbero et al., 2002). Late endosome acts as the last sorting station from where proteins can be directed to lysosomes for degradation (Gruenberg, 2001), or retrieved and delivered to the trans Golgi network (Maxfield and McGraw, 2004), or to the plasma membrane (Stoorvogel et al., 2002; Trombetta and Mellman, 2005). Some degradation can also occur in late endosomes (van der Goot and Gruenberg, 2006).

The degradation pathway involves the delivery of the content from late endosomes to lysosomes, which is an exchange process taking place both by fusion and 'kiss-and-run' interaction (Bright et al., 2005). It is difficult to distinguish between late endosomes and lysosomes using light microscopy, as they share most of the marker proteins. Nevertheless, lysosomes can be identified by electron-microscopy due to their morphological features, such as 
the electron-dense appearence, but also by biochemical methods which reveal their specific physical properties on gradients (Gruenberg, 2001).

2.2.4. Communication of late stations of the endocytic pathway with the plasma membrane

Lysosomes represent a termination point for most, but not all proteins. There are many studies reporting that late endosomes and lysosomes can become mobile and deliver their material to the plasma membrane and extracellular environment. One example of the so-called retrograde transport from late endosomes/lysosomes to the plasma membrane is the translocation of the major histocompatibility ( $\mathrm{MHC}$ ) class II to the cell surface during the maturation of dendritic cells (Trombetta and Mellman, 2005). Some cell types, including cells derived from the hematopoietic lineage (Blott and Griffiths, 2002), and melanocytes (Marks and Seabra, 2001) contain secretory lysosomes, also known as lysosome-related organelles. Secretory lysosomes are specialized, dual-function organelles which are, in addition to their degradative function, able to store and release newly synthesized secretory proteins. The biochemical composition of these organelles is very similar to that of the conventional lysosomes, but it comprizes an additional cell-typespecific set of secreted constituents (Blott and Griffiths, 2002).

There are currently two hypotheses about the evolution of secretory lysosomes. The first one is that the secretory lysosomes have evolved from conventional lysosomes which acquired the ability to secrete their content. This idea is supported by the fact that these organelles share many common characteristics. The second hypothesis is that they might be distantly related to secretory granules, storage compartments in specialized secretory cells (Blott and Griffiths, 2002). Immature granules, the precursors of the secretory granules, are acidic and contain both lysosomal and secretory proteins (Klumperman et al., 1998; Kuliawat et al., 1997).

Exocytosis of the secretory lysosomes is a regulated process, as exemplified in cytotoxic T-lymphocyte: the binding of a cell-surface receptor acts as a signal inducing $\mathrm{Ca}^{2+}$ mobilization within the cell, leading eventually 
to the mobilization of secretory lysosomes for degranulation (Lyubchenko et al., 2001). These organelles move along microtubules, driven by a motor from the kinesin family (Burkhardt et al., 1993). Once near the cell periphery, lysosomes use actin-based movement to reach their final docking site on the plasma membrane (Langford et al., 1994). The process of exocytosis requires a common cellular machinery that is shared with secretory granules, including synaptotagmins, proteins that bind phospholipid membranes in $\mathrm{Ca}^{2+}$ dependent manner (Martinez et al., 2000) and part of the SNARE fusion machinery (Paumet et al., 2000). Additionally, secretory lysosomes seem to use some unique components, like Rab27a, a specific member of the Rab family small GTPases (regulators of budding, tethering, fusion and motility at various cellular sites) and other factors, such as Lyst (Blott and Griffiths, 2002).

Interestingly, not only specialized lysosomes, but also conventional lysosomes have the ability to fuse with the plasma membrane under certain conditions (Andrews, 2000). This process seems to play a role in plasma membrane repair, for example during wound healing (Andrews, 2002).

\subsubsection{Possible fates of intralumenal vesicles of late endosome}

Late endosomes can fuse with lysosomes, thereby delivering their ILVs for degradation, a process used for the downregulation of receptors (Futter et al., 1996). Alternatively, the late endosome can fuse with the plasma membrane, thereby releasing the ILVs into extracellular environment as exosomes (Stoorvogel et al., 2002). Exosomes are small membrane vesicles with a diameter of $40-100 \mathrm{~nm}$, that are secreted by many cell types into the extracellular environment and may play a role in different forms of intercellular communications (Stoorvogel et al., 2002). Another possibility is the so-called back-fusion of ILVs with the endosomal limiting membrane, which prevents both lysosomal degradation and exosome release. Back-fusion has been suggested to play a role in the retrieval of MHC-II from ILVs during the maturation of dendritic cells (Boes et al., 2002; Chow et al., 2002; Kleijmeer et al., 2001). It is also used by pathogens for escaping the lysosomal 
degradation, as it allows their entry into the cytoplasm of infected cells. Prime examples are the antrax virus (Abrami et al., 2004) and vesicular stomatitis virus (VSV) (Le Blanc et al., 2005). In the dendritic cells, the back-fusion of ILVs is followed by the formation of tubulovesicles which can extend and fuse with the plasma membrane, thereby delivering the endosomal membranes to the cell surface (Kleijmeer et al., 2001). 
3. Results 


\subsection{Publication 1:}

Neuron to glia signaling triggers myelin membrane endocytosis from endosomal storage sites 


\title{
Neuron to glia signaling triggers myelin membrane exocytosis from endosomal storage sites
}

\author{
Katarina Trajkovic, ${ }^{1,2}$ Ajit Singh Dhaunchak, ${ }^{2}$ José T. Goncalves, ${ }^{2}$ Dirk Wenzel, ${ }^{1}$ Anja Schneider, ${ }^{1,2}$ Gertrude Bunt, ${ }^{2}$ \\ Klaus-Armin Nave, ${ }^{2}$ and Mikael Simons ${ }^{1,2}$ \\ 'Centre for Biochemistry and Molecular Cell Biology, University of Göttingen, 37073 Göttingen, Germany \\ ${ }^{2}$ Max-Planck-Institute for Experimental Medicine, 37075 Göttingen, Germany
}

D uring vertebrate brain development, axons are enwrapped by myelin, an insulating membrane produced by oligodendrocytes. Neuron-derived signaling molecules are temporally and spatially required to coordinate oligodendrocyte differentiation. In this study, we show that neurons regulate myelin membrane trafficking in oligodendrocytes. In the absence of neurons, the major myelin membrane protein, the proteolipid protein (PLP), is internalized and stored in late endosomes/lysosomes (LEs/Ls) by a cholesterol-dependent and clathrin- independent endocytosis pathway that requires actin and the RhoA guanosine triphosphatase. Upon maturation, the rate of endocytosis is reduced, and a CAMPdependent neuronal signal triggers the transport of PLP from $L E s / L s$ to the plasma membrane. These findings reveal a fundamental and novel role of LEs/Ls in oligodendrocytes: to store and release PLP in a regulated fashion. The release of myelin membrane from LEs/Ls by neuronal signals may represent a mechanism to control myelin membrane growth.

\section{Introduction}

The myelin sheath is a multilamellar, spirally wrapping extension of the plasma membrane of oligodendrocytes that is essential for rapid impulse conduction in the central nervous system. This specialized membrane exhibits a unique composition with $>70 \%$ of the dry weight consisting of lipids and the remainder being comprised of a restricted set of proteins, of which most are exclusively found in myelin (Baumann and Pham-Dinh, 2001; Kramer et al., 2001). The major central nervous system myelin proteins, the myelin basic protein, and the proteolipid proteins (PLPs/DM20) are low molecular weight proteins found in compact myelin that constitute $80 \%$ of the total myelin proteins. PLP is a highly hydrophobic protein with four transmembrane domains that interact with cholesterol and galactosylceramideenriched membranes during its biosynthetic transport in oligodendrocytes (Weimbs and Stoffel, 1992; Simons et al., 2000; Schneider et al., 2005).

To form the myelin sheath, oligodendrocytes must deliver large amounts of myelin membrane to the axons at the appropriate developmental stage of the oligodendroglial and neuronal

K. Trajkovic and A.S. Dhaunchak contributed equally to this paper.

Correspondence to M. Simons: msimons@gwdg.de

Abbreviations used in this paper: $L E / L$, late endosome/lysosome; $m \beta C D$, methyl$\beta$-cyclodextrin; MHC, major histocompatibility complex; PLP, proteolipid protein; TIRFM; total internal reflection fluorescence microscopy.

The online version of this article contains supplemental material. cell lineage (Baumann and Pham-Dinh, 2001; Kramer et al., 2001). On the other hand, axons produce signals that regulate the differentiation of oligodendrocytes (Barres and Raff, 1999; Fields and Stevens-Graham, 2002). This led us to postulate that neuronal signals could be involved in the coordination of the trafficking of myelin membrane in oligodendrocytes. In this study, we show that the transport of PLP in oligodendrocytes is under neuronal control. PLP is initially targeted to late endosomes/lysosomes (LEs/Ls) by using a cholesterol-dependent and clathrin-independent endocytosis pathway. PLP is then redistributed from LEs/Ls to the plasma membrane upon activation by neuronal cells. We provide evidence that this developmentdependent regulation of PLP localization occurs by the downregulation of endocytosis and by the stimulation of exocytosis from LE/L storage sites.

\section{Results}

\section{PLP localizes to LEs/Ls}

\section{of immature oligodendrocytes}

To analyze the localization of PLP in immature oligodendrocytes, oligodendroglial precursor cells growing on top of a layer of astrocytes were shaken off and cultured for $3 \mathrm{~d}$ to induce the expression of PLP. By confocal immunofluorescence microscopy, extensive colocalization of PLP and Lamp-1, a marker for 
a
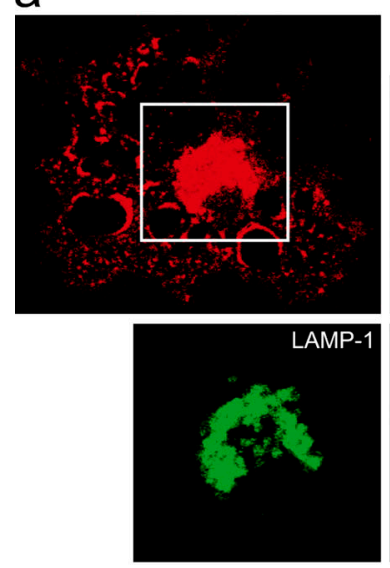

C
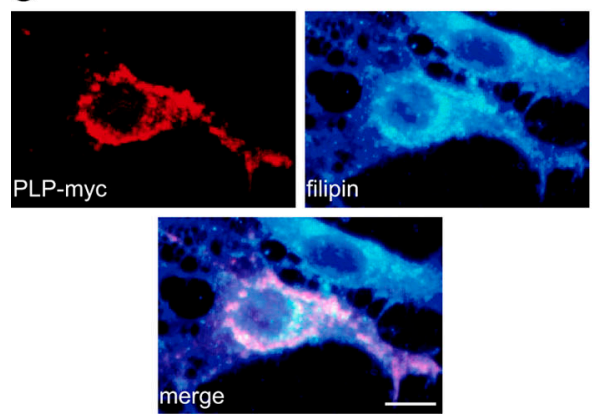

b
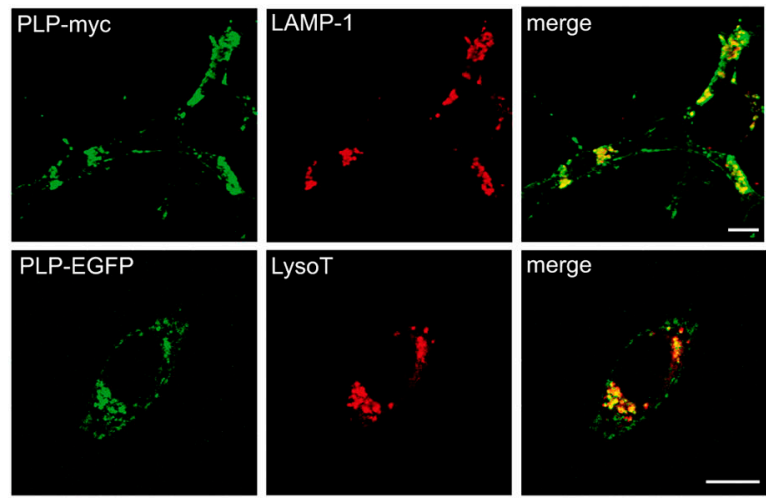

d
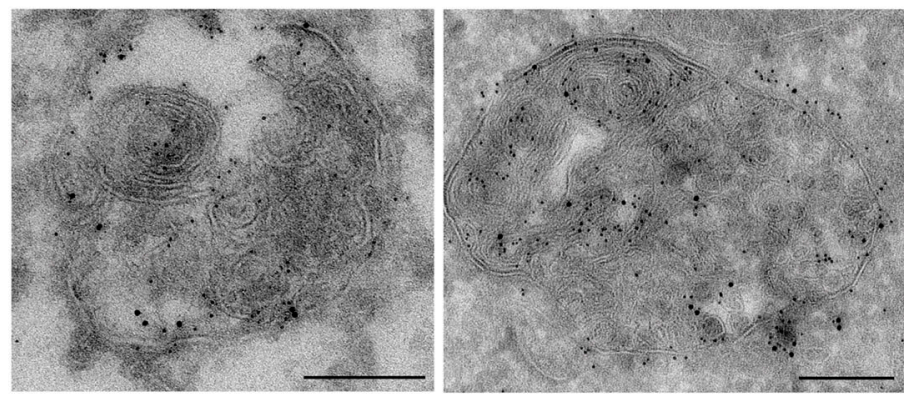

Figure 1. PLP localizes to LEs/Ls of immature oligodendrocytes. (a) Confocal immunofluorescence microscopy demonstrates the colocalization of endogenous PLP (red) with Lamp-1 (green) in primary oligodendrocytes grown for $3 \mathrm{~d}$ in vitro. The region in the boxed area is shown at higher magnification and lower exposure. (b) Top panels show the colocalization of PLP-myc (green) with Lamp-1 (red) in Oli-neu cells, and the bottom panels show the colocalization of PLP-EGFP (green) with LysoTracker red in living OLN-93 cells. (c) Filipin staining reveals colocalization of cholesterol (blue) and PLP (red) in OLN-93 cells. (a-c) Bars, $5 \mu \mathrm{m}$. (d) Immuno-EM double labeling of primary oligodendrocytes (left) and Oli-neu cells (right) with antibodies directed against Lamp-1 (5 nm gold) and against PLP, polyclonal P6 (left), or GFP (right; both $10 \mathrm{~nm}$ gold) shows the localization of PLP in Lamp-1-containing multivesicular and multilamellar compartments. Bars, $200 \mathrm{~nm}$.

LEs/Ls, was observed (Fig. 1 a) as reported previously (Kramer et al., 2001; Simons et al., 2002). The same striking colocalization of PLP and Lamp-1 was observed in an immortalized oligodendroglial precursor cell line, Oli-neu. Fusion of either a myc tag or EGFP to PLP did not affect the LE/L targeting of PLP (Fig. 1 b). To obtain further support for the localization of PLP to LEs/Ls in immature cells, we used a spontaneously transformed oligodendroglial precursor cell line, OLN-93. When these cells were incubated for $5 \mathrm{~h}$ with rhodamine-dextran followed by a 2-h chase or were treated with LysoTracker red DND-99 to stain for LEs/Ls, colocalization with PLP was observed (Fig. $1 \mathrm{~b}$ and not depicted). Staining with filipin revealed a partial colocalization of PLP with cholesterol in LEs/Ls (Fig. 1 c).
To resolve the ultrastructure of the PLP-containing organelles, we performed immunoelectron microscopy (Fig. $1 \mathrm{~d}$ ). Both endogenous PLP and PLP-EGFP colocalized with Lamp-1 in vacuolar structures that contained abundant lumenal multilamellar and/or multivesicular membrane arrays.

\section{PLP disappears from LEs/Ls when oligodendrocytes are cocultured with neurons}

To determine whether the subcellular localization of PLP is influenced by the presence of neuronal cells, oligodendroglial progenitor cells were added to a neuronal cell culture. Similar to the cultures without neurons, oligodendrocytes started to

Figure 2. PLP disappears from LEs/Ls when oligodendrocytes are cocultured with neurons. (a) Primary oligodendrocytes were grown for 3 or $5 \mathrm{~d}$ with or without neurons. Confocal microscopy analysis of PLP (red) and Lamp-1 (green) demonstrates a depletion of PLP from LEs/Ls in oligodendrocytes when cultured in the presence of neurons for $5 \mathrm{~d}$. Axons are visualized by $\beta$ lll-tubulin staining (blue). (b) Oli-neu cells were grown for $2 \mathrm{~d}$ with (bottom) or without (top) neurons. PLP-EGFP (green) disappears from Lamp-l-containing compartments (red) by $2 \mathrm{~d}$ of coculture with neurons. (a and b) The regions in the boxed areas are shown at a higher magnification and lower exposure. (c) Surface staining of living cells with $010 \mathrm{mAb}$ at $4^{\circ} \mathrm{C}$ shows that the majority of PLP-EGFP is found at the surface of the cell in a coculturing with neurons. Quantitative analysis of the colocalization of PLP-EGFP with Lamp-1 (b) and O10 (c) are shown. Error bars represent SD ( $n>30$ cells). (a-c) Bars, $5 \mu \mathrm{m}$. (d) Immunohistochemistry of brain sections of P7 and P60 adult mice for PLP (red) and Lamp-1 (green). Colocalization was observed in sections from P7 but not adult mice. Bars, $10 \mu \mathrm{m}$. (e) Oligodendrocytes (for $2 \mathrm{~d}$ in culture) grown with or without neurons were metabolically labeled with ${ }^{35}$ S $]$ methionine/cysteine for $18 \mathrm{~h}$ and chased for $2 \mathrm{~d}$ (day 5) or not chased (day 3 ) before performing the PLP immunoprecipitations. Quantitative analysis of three independent experiments did not reveal any significant differences in the amount of labeled PLP and its alternatively spliced isoform DM20 (mean $\pm \mathrm{SD}) .{ }^{* *}, \mathrm{P}<0.001 ; t$ test. 
a
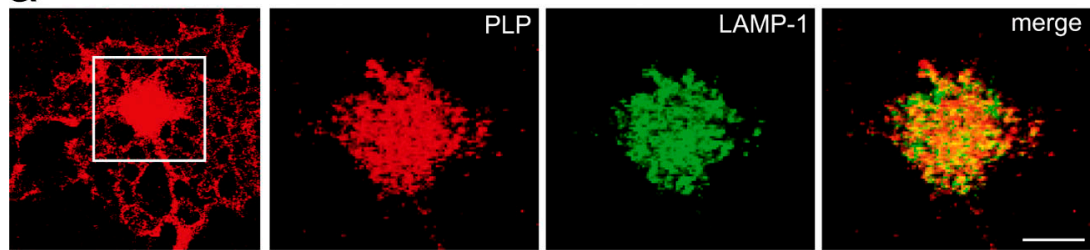

day 5

pOl
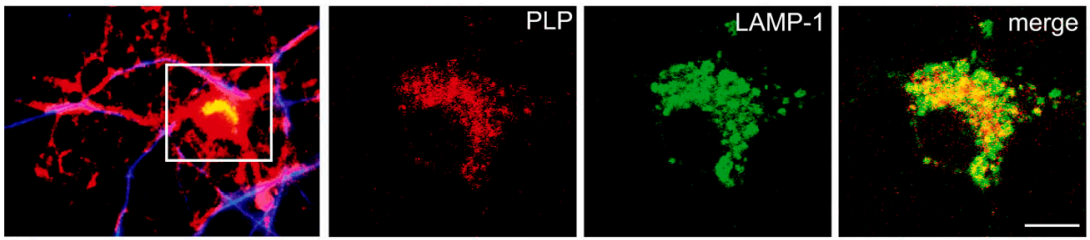

day 3

$\mathrm{pOl} / \mathrm{neurons}$
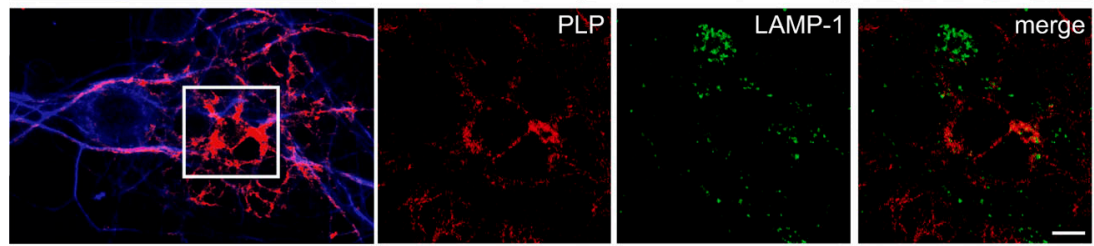

day 5

$\mathrm{pOl} / \mathrm{neurons}$

b
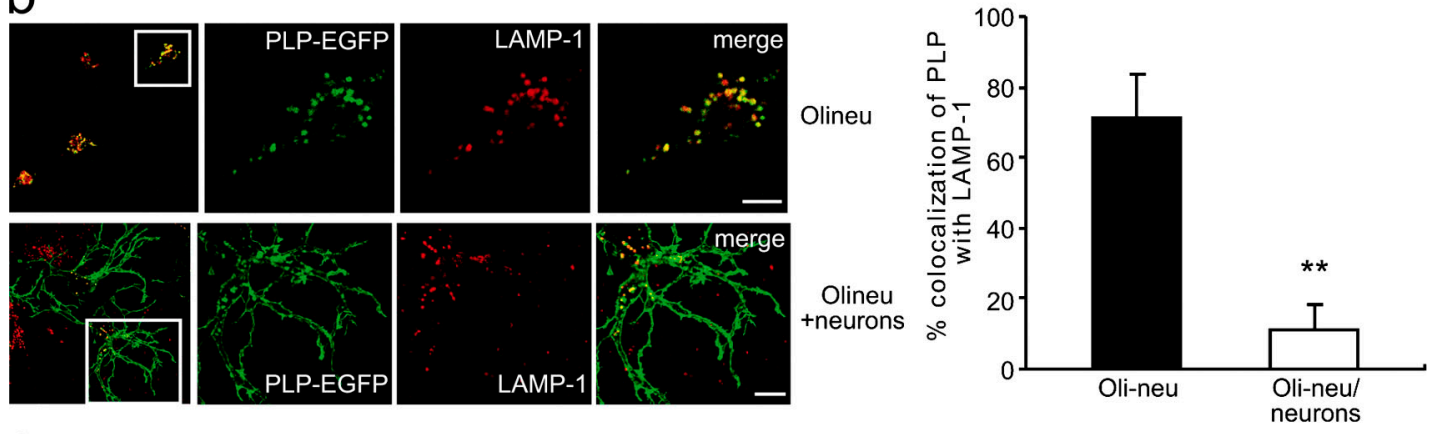

C
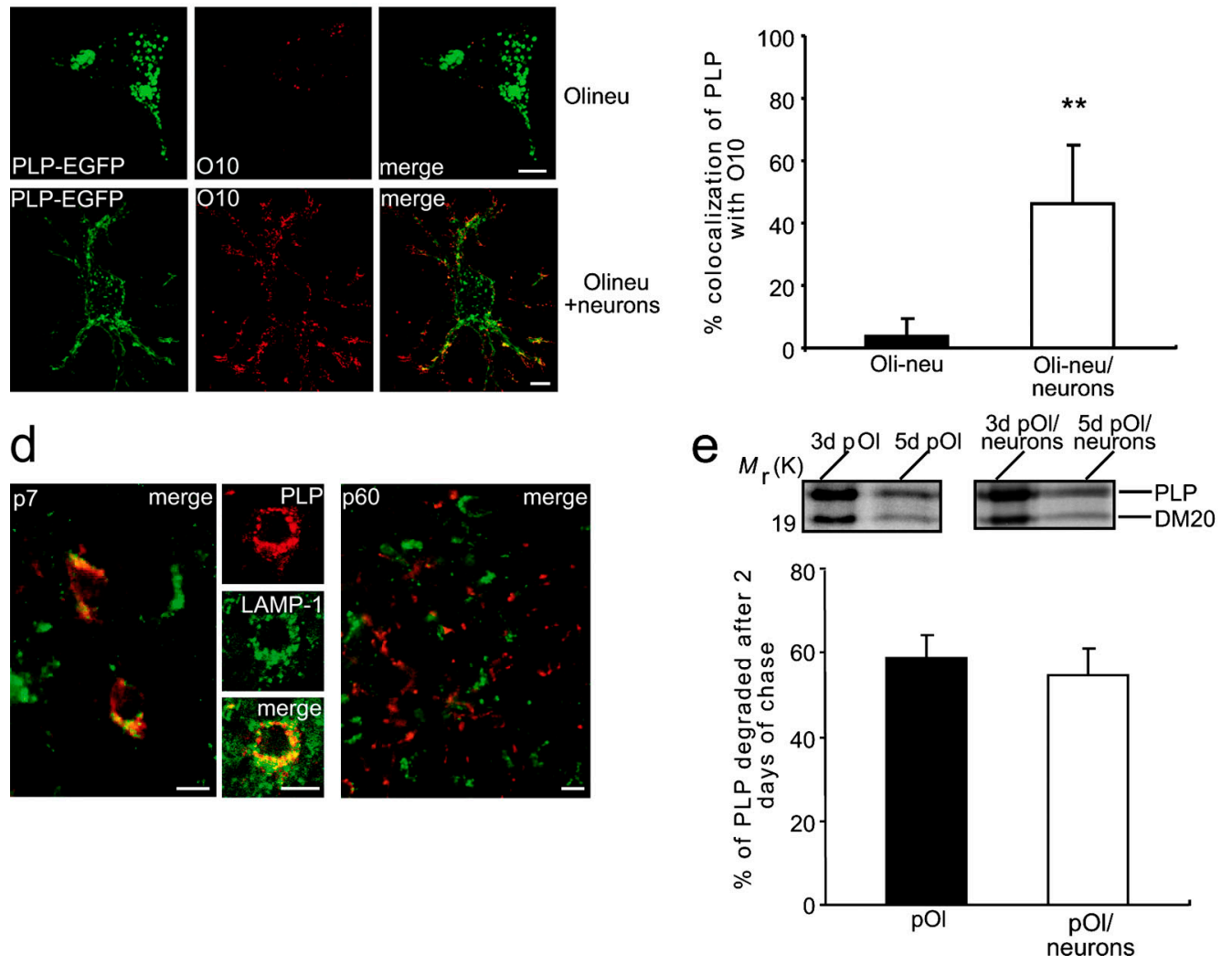

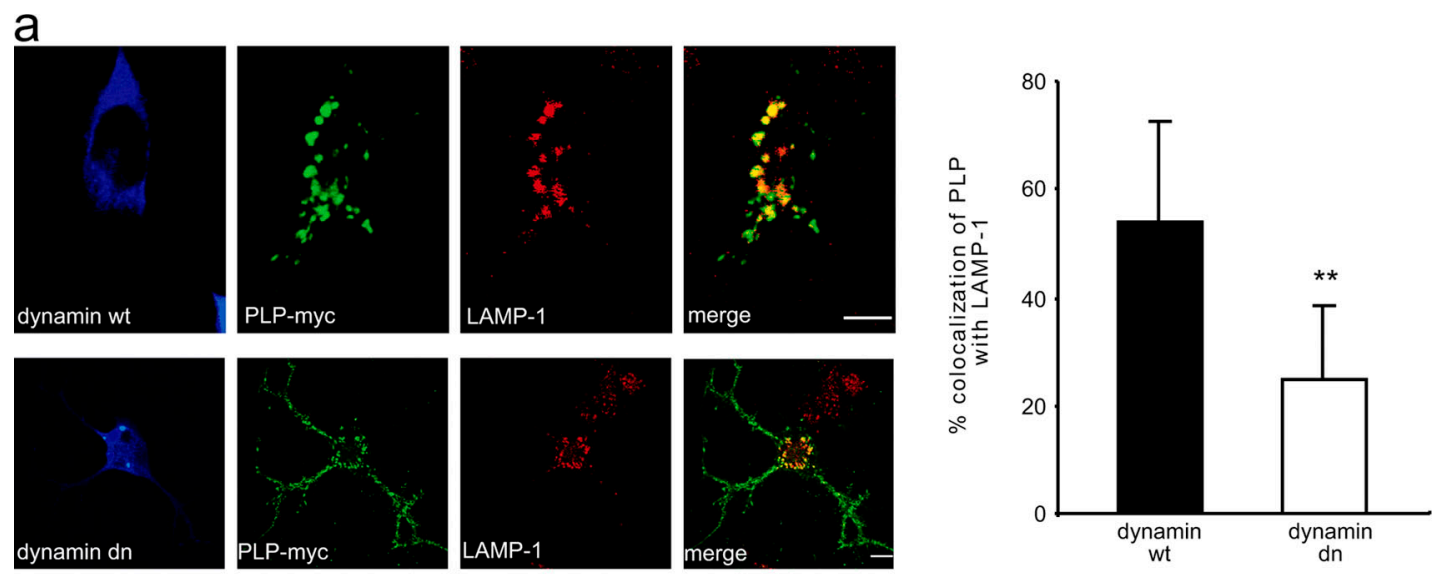

b
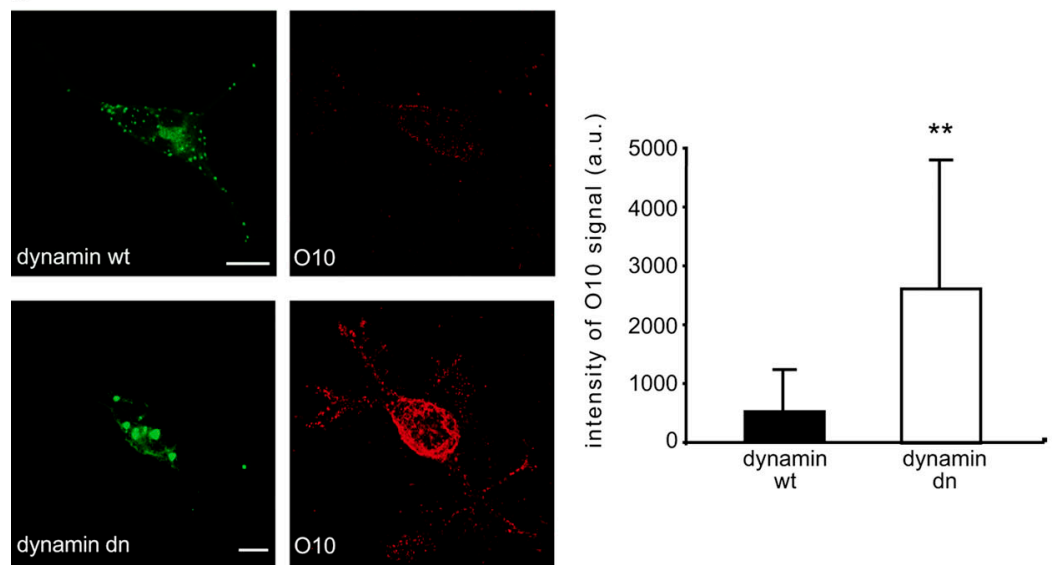

Figure 3. PLP is routed to LEs/Ls by endocytosis. Oli-neu cells were cotransfected with PLP-myc and wild-type or dominant-negative dynamin-II (K44A, dynamin dn), both containing an HA tag, and were stained for Lamp-1 (a) or for surface PLP (b) with $\mathrm{O} 10 \mathrm{mAb}$ (at $4^{\circ} \mathrm{C}$ on living cells). (a) Quantitative analysis of the colocalization of PLP-EGFP with Lamp-1 is shown. (b) Quantitative analysis of the fluorescence intensity of O10 signal is shown in arbitrary units (a.u.) per cell. ( $a$ and b) Values are given as the means \pm SD (error bars) of a mean of three independent experiments with $>40$ (a) or $>30$ (b) cells. **, $\mathrm{P}<0.001 ; t$ test. Bars, $5 \mu \mathrm{m}$.

express PLP during the first $2 \mathrm{~d}$ in culture, and an accumulation of PLP in LEs/Ls was observed in $~ 90 \%$ of the cells after $3 \mathrm{~d}$ in culture (Fig. 2 a). However, in marked contrast to cultures without neurons, we observed that PLP disappeared from LEs/Ls $2 \mathrm{~d}$ later (only $\sim 20 \%$ of the cells showed an accumulation of PLP in LEs/Ls) in the presence of neurons (Fig. 2 a). Also, the staining of Lamp-1-containing structures decreased in intensity after PLP removal. To follow the developmental regulation of PLP trafficking in Oli-neu cells, we produced PLP-EGFP-stably expressing cell lines. Fusion of EGFP to PLP did not interfere with transport to the cell surface, as indicated by the positive staining of transfected Oli-neu cells with O10 mAb. This antibody recognizes a conformation-dependent epitope of PLP on the surface of living cells (Jung et al., 1996). In addition, transfection of primary myelinating oligodendrocytes confirmed that PLP-EGFP is transported to myelin (Fig. S1, available at http://www.jcb.org/cgi/content/full/jcb.200509022/DC1). When PLP-EGFP-expressing Oli-neu cells were added on top of a neuronal culture, a dramatic change in the localization of PLP was observed (Fig. 2 b). Quantitative analysis showed that $71.5 \%$ of PLP colocalized with Lamp-1 in Oli-neu cells alone, whereas only $11.5 \%$ of PLP colocalized with Lamp-1 in cells that had been in coculture with neurons for $2 \mathrm{~d}$ (Fig. $2 \mathrm{~b}$ ). Moreover, surface staining of living cells at $4^{\circ} \mathrm{C}$ with $\mathrm{O} 10 \mathrm{mAb}$ showed that the majority of PLP-EGFP was located at the plasma membrane in cells cultured with but not without neurons (Fig. 2 c). To test whether the localization of PLP shows the same developmental regulation in vivo, we performed immunohistochemistry on brain sections of young (postnatal day [P] 7) and adult mice (P60). Significant colocalization of PLP and Lamp-1 was only observed in cells of P7 mice but not in sections prepared from adult mice (Fig. 2 d). Analysis of the sections indicated that the colocalization of PLP and Lamp-1 was increased $>20$-fold in $\mathrm{P} 7$ mice as compared with adult mice. Together, our data demonstrate that PLP disappears from LEs/Ls upon oligodendroglial maturation and emerges at the surface of the oligodendrocyte in a process that is dependent on the presence of neuronal cells.

There are several possibilities to explain our results. One possibility is that less PLP is transported into and/or more PLP is transported out of LEs/Ls in the presence of neurons. An alternative explanation is that the degradation of PLP in lysosomes increases during development. To test the latter hypothesis, we performed pulse-chase experiments. Primary oligodendrocytes 
a
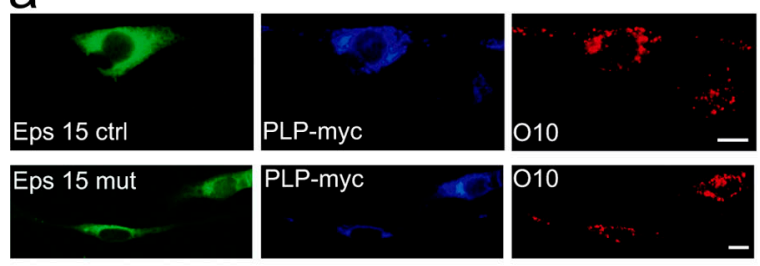

b
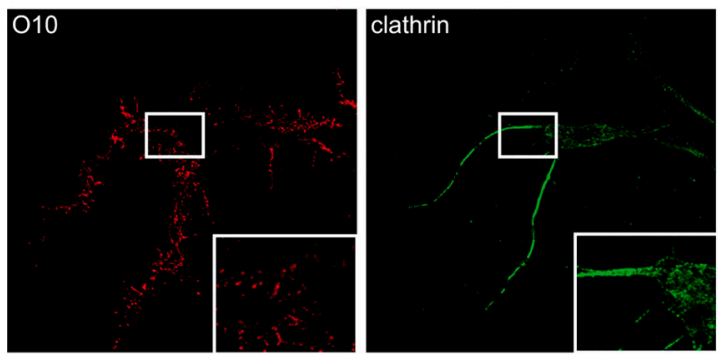

C
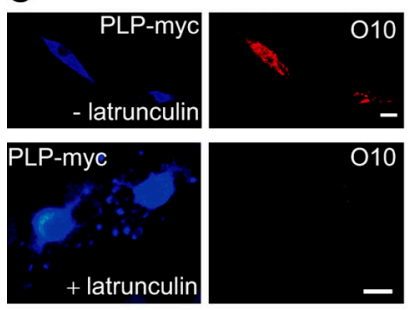

e
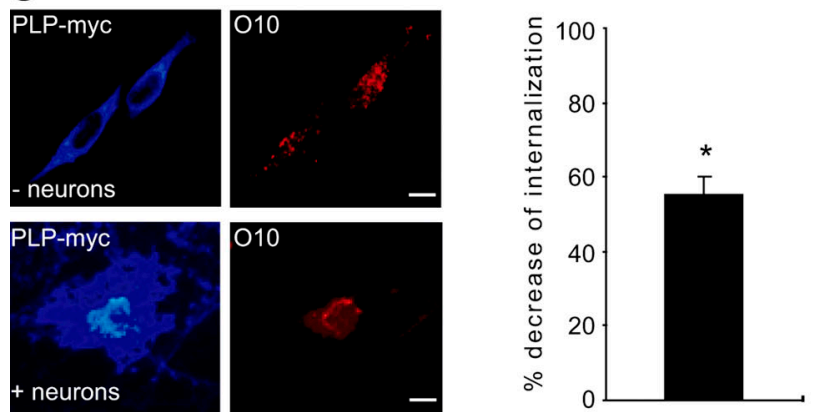
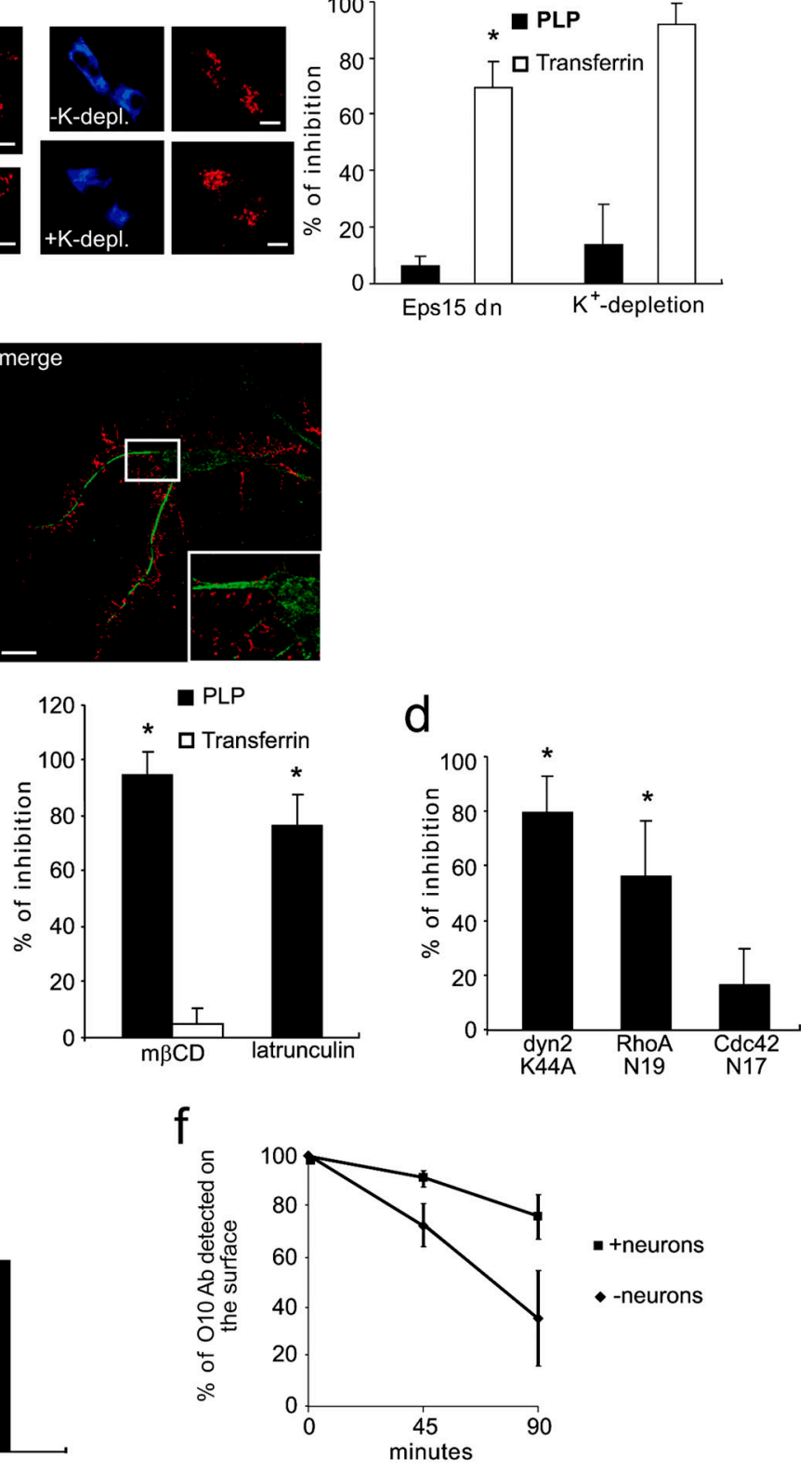

Figure 4. The cholesterol-dependent and clathrin-independent internalization of PLP is reduced after coculturing with neurons. Antibody uptake experiments were performed with the PLP antibody O10 followed by an acidic wash to remove surface-bound antibody. The internalized antibody is shown in red. (a) OLN-93 cells were cotransfected with PLP (blue) and with Eps $15 \mathrm{III} \Delta 2$ (Eps 15 ctrl) or Eps 15 E $495 / 295$ (Eps 15 mut; green). Potassium depletion was used as an alternative approach to interfere with clathrin function. Clathrin-mediated transferrin-rhodamine uptake served as a control. The amount of inhibition was evaluated by determining the fluorescence intensities in comparison with the control conditions. Values are given as the mean \pm SD (error bars) of a mean of three independent experiments with $>50$ cells. (b) PLP stained with O10 (red) on the surface of OLN-93 cells does not show a significant colocalization with clathrin heavy chain (green). Insets are shown at higher magnification in the right corner of the images. (c) OLN-93 cells were transfected with PLP (blue) followed by a 30-min treatment with $1 \mu \mathrm{M}$ latrunculin to inhibit the polymerization of the actin cytoskeleton or by a 15 -min incubation with $5 \mathrm{mM} \mathrm{m} \beta C D$ to deplete cholesterol before performing the 010 internalization assay. Transferrinrhodamine was used as a control for the cholesterol depletion experiments. Inhibition of the 010 uptake is shown as the mean \pm SD of three independent experiments with $>50$ cells. (d) Cells were cotransfected with PLP and with wild type or the respective dominant-negative variants of dynamin-II, RhoA, and cdc42. The inhibition of $\mathrm{O} 10$ antibody uptake by the dominant-negative protein is shown in comparison with the respective wildtype construct as the mean \pm SD of three independent experiments with $>40$ cells. The inhibition of fluid phase endocytosis (measured by dextran uptake) served as a positive control for dominant-negative cdc42 (not depicted). (e) OLN-93 cells were transfected with PLP-myc and added on top of a neuronal culture or left alone. O10 antibody uptake experiments were performed followed by an acidic wash to remove surface-bound antibody. The amount of inhibition is shown for OLN-93 cells cultured with neurons compared with cells cultured without neurons as the mean \pm SD of three experiments with $>40$ cells. (f) The reduction of cell surface PLP was determined after various times in culture with or without neurons. Cell surface PLP was labeled with $010 \mathrm{mAb}$ at $4^{\circ} \mathrm{C}$, and incubation was continued at $37^{\circ} \mathrm{C}$ for 0,45 , and $90 \mathrm{~min}$. The amount of cell surface remaining $\mathrm{O} 10 \mathrm{mAb}$ was analyzed by incubating cells with $\left[{ }^{125} \mid\right.$ ]-labeled secondary antibody at $4^{\circ} \mathrm{C}$ and subsequent determination of the radioactivity by $\gamma$ counting. Quantification is shown as the mean $\pm S D$ of three experiments. ${ }^{*}, \mathrm{P}<0.01 ; t$ test. Bars, $5 \mu \mathrm{m}$. 
(for $2 \mathrm{~d}$ in culture) grown with or without neurons were metabolically labeled with $\left[{ }^{35} \mathrm{~S}\right]$ methionine/cysteine for $18 \mathrm{~h}$ and chased for $2 \mathrm{~d}$. Hence, the metabolic labeling was performed at the stage of development (day 2-3) when PLP accumulates in LEs/Ls. The cells were chased up to the developmental stage (day 5) when PLP is almost completely removed from LEs/Ls in cells cultured with neurons but not in cells cultured without. Nevertheless, we did not observe any significant differences in the amount of labeled PLP and its alternatively spliced isoform DM20 (Fig. 2 e). Thus, differential proteolysis of PLP does not seem to be the underlying reason for the removal of PLP from LEs/Ls. Therefore, it is more likely that neuronal signals influence the transport of PLP into and/or out of LEs/Ls in oligodendrocytes.

The cholesterol-dependent and clathrin-independent internalization of PLP is reduced after coculturing with neurons

Next, we determined whether endocytosis accounts for the transport of PLP to LEs/Ls and, if so, how its endocytic trafficking is regulated. To block endocytosis, we transiently transfected Oli-neu cells with a mutant form of dynamin-II that is defective in GTP binding (K44A; Damke et al., 1994) and PLP-myc. We found that dynamin-II (K44A) reduced the colocalization of PLP with Lamp-1 and, at the same time, increased the fraction of PLP at the cell surface (Fig. 3), suggesting that endocytosis is required for the transport of PLP to LEs/Ls. A reduction of the intracellular accumulation of PLP was also observed when dynamin K44A was expressed in OLN-93 cells (not depicted).

To gain more insight into the endocytosis process of PLP, the endocytic uptake of PLP was determined by additional antibody uptake experiments using the $\mathrm{O} 10 \mathrm{mAb}$. To determine whether clathrin function is involved, we either depleted cells of potassium to disrupt the formation of clathrin-coated pits or used the dominant-negative mutant of Eps15 (E $\Delta 95 / 295$; Benmerah et al., 1999). Despite that E $\Delta 95 / 295$ and $\mathrm{K}^{+}$depletion had no significant effect on the uptake of $\mathrm{O} 10$, it led to the reduction of clathrin-mediated transferrin-rhodamine internalization (Fig. 4 a). In addition, intracellular accumulation of PLP was not reduced when E $\Delta 95 / 295$ was coexpressed in OLN-93 (not depicted). Furthermore, we did not detect a significant colocalization of PLP and clathrin on the surface of OLN-93 (colocalization was $\sim 5 \%$; Fig. 4 b) or primary oligodendroglial cells (Fig. S2; available at http://www.jcb.org/cgi/content/full/ jcb.200509022/DC1). These results strongly suggest that the internalization of PLP occurs by a clathrin-independent endocytosis pathway.

Most clathrin-independent endocytosis pathways are sensitive to cholesterol depletion or actin depolymerization (Parton and Richards, 2003). Therefore, we used methyl- $\beta$-cyclodextrin $(\mathrm{m} \beta C D)$ to selectively extract cholesterol from the cell surface and latrunculin A to prevent actin polymerization. Treatments with either $m \beta C D$ or latrunculin A led to an almost complete inhibition of O10 uptake (Fig. 4 c). The conditions of the cholesterol depletion experiments were established so that clathrindependent endocytosis was not affected as evaluated by the uptake of transferrin-rhodamine. Some clathrin-independent endocytosis pathways require dynamin, whereas others are independent of dynamin function (Lamaze et al., 2001; Pelkmans et al., 2001; Sabharanjak et al., 2002; Damm et al., 2005; Kirkham et al., 2005). The uptake of $\mathrm{O} 10$ was clearly reduced by interfering with dynamin function (Fig. $4 \mathrm{~d}$ ), which is consistent with the redistribution of PLP to the cell surface by dynamin-II (K44A; Fig. 3). Because both cholesterol and dynamin are essential for caveolar-dependent uptake, we compared the localization of PLP to caveolin-1 and GFP-caveolin. Caveolin-1 and GFP-caveolin were detected in punctate arrays on the plasma membrane and on intracellular compartments, but no colocalization with PLP was seen (Fig. S2 and not depicted), suggesting that caveolae are not involved in the endocytosis of PLP. The Rho family of small GTPases differentially regulate nonclathrin and noncaveolar endocytosis pathways. Although cdc42 is involved in the endocytosis of glycosyl-phosphatidylinositol-anchored proteins by a pinocytic pathway to recycling endosomes (Sabharanjak et al., 2002), rhoA has been implicated in the dynamin-dependent uptake of interleukin 2 receptor to LEs/Ls (Lamaze et al., 2001). When O10 uptake experiments were performed with dominant-negative mutants of either cdc42 or rhoA, we observed a significant reduction of internalization when the function of rhoA but not cdc42 was inhibited (Fig. 4 d). In summary, our results show that OLN-93 cells use a clathrin-independent but cholesterol-dependent endocytosis pathway that requires a functional actin cytoskeleton and the rhoA GTPase.

To test whether the capacity for endocytosis of PLP changes after contact with neurons, we added OLN-93 cells to neuronal cultures and compared the uptake of $\mathrm{O} 10$ into cells that were cultured without neurons. We observed a significant reduction in the internalization of PLP in cells in coculture as compared with cells cultured without neurons (Fig. 4 e). We also analyzed the reduction of cell surface PLP upon various times in culture with or without neurons using the $010 \mathrm{mAb}$ internalization assay. The amount of $\mathrm{O} 10 \mathrm{mAb}$ remaining at the cell surface was analyzed by incubating cells with $\left[{ }^{125} \mathrm{I}\right]$-labeled secondary antibody at $4{ }^{\circ} \mathrm{C}$. We found that PLP was cleared more efficiently over time from the surface of OLN-93 cells that were cultured without neurons as compared with cells in coculture. Together, these results indicate that neurons reduce the endocytosis of PLP (Fig. 4 f).

Neurons trigger the retrograde transport of PLP from LEs/Ls to the surface of oligodendrocytes

Reduction of endocytosis appears to be one reason why PLP disappears from LEs/Ls after contact with neurons. Another event that could simultaneously contribute is the increased resorting of PLP to the plasma membrane by retrograde transport from LEs/Ls. There are many examples (e.g., wound healing, cytotoxic lymphocyte killing, major histocompatibility complex [MHC]-II processing, and melanin secretion) that show that lysosomes are not merely degradative dead ends but are able to store and release proteins in a regulated fashion (Blott and Griffiths, 2002).

To analyze the putative exocytic trafficking of PLP from LEs/Ls, we performed live cell imaging experiments with 

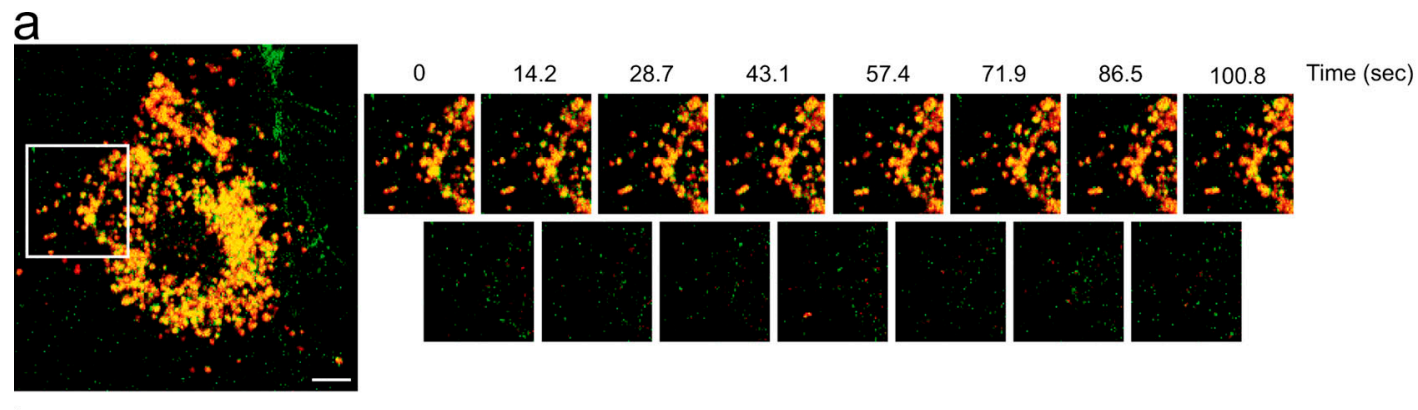

b
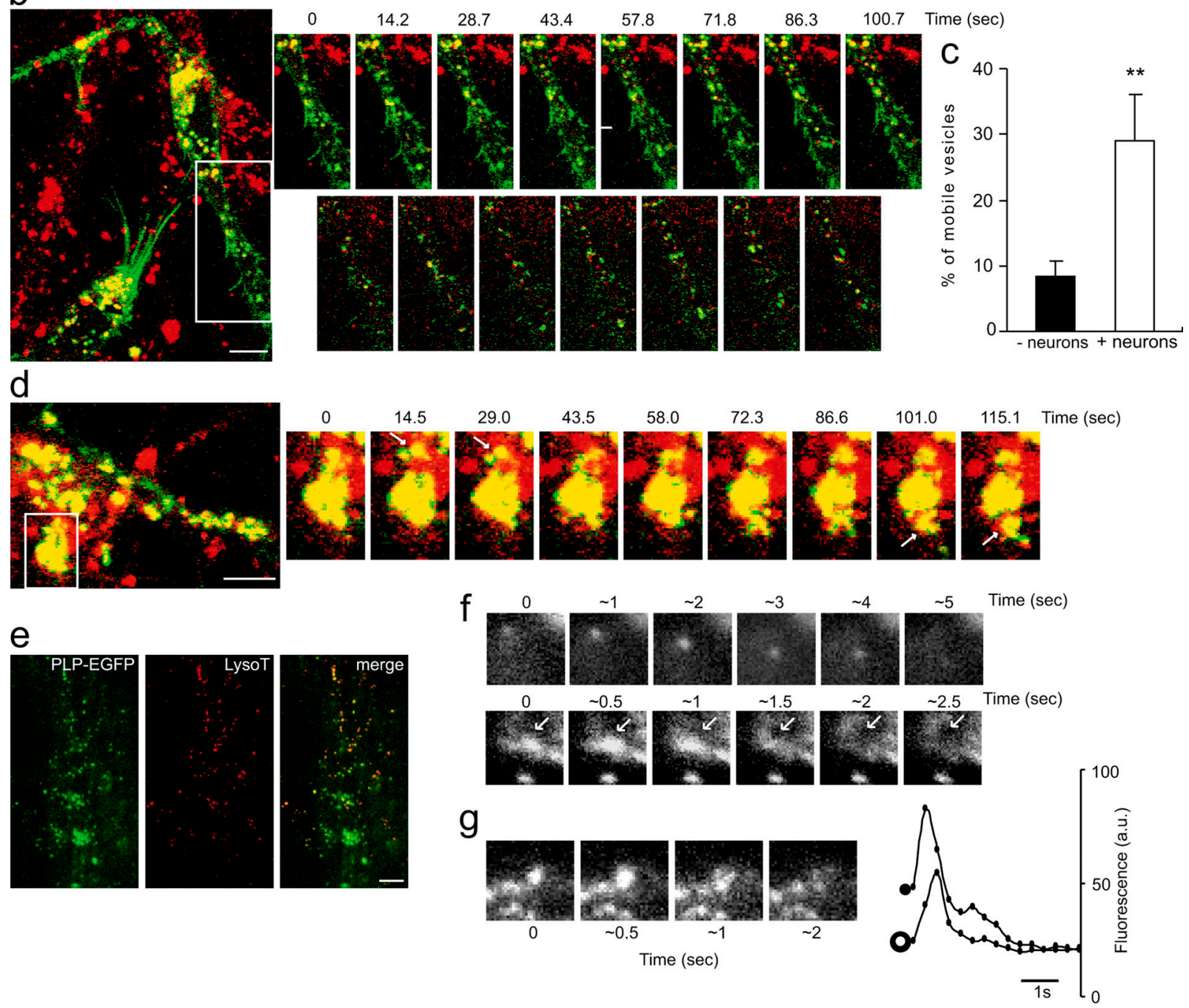

Figure 5. Neurons trigger the retrograde transport of PLP from LEs/Ls to the surface of oligodendrocytes. (a) Living PLP-EGFP-expressing Oli-neu cells were labeled with LysoTracker red and observed by confocal microscopy. Images were collected every $\sim 15 \mathrm{~s}$. The image sequences of the boxed areas $(a, b$, and d) are shown. Representative examples of a time stack are shown in the top panels. The images in the bottom panels were obtained by subtracting from each image in the top panels. The absence of signal in the subtractions demonstrates the immobility of vesicles. (b) Cells were added onto a neuronal culture and imaged $\sim 8 \mathrm{~h}$ later. The subtractions (bottom) of consecutive frames (top) demonstrate the mobility of PLP and LysoTracker-containing vesicles. (c) The mean fraction of mobile vesicles calculated by the subtraction of consecutive frames (mean \pm SD [error bars] of 15 cells; 26 consecutive time frames were analyzed for each cell). ${ }^{* *}, \mathrm{P}<0.001 ; t$ test. (d) Individual frames from a video of a cell prepared as in $\mathrm{b}$. Images were taken every $\sim 15 \mathrm{~s}$. The frame sequence illustrates the exit (indicated by arrows) of PLP and LysoTracker-containing vesicles from perinuclear LEs/Ls. (e) The TIRFM image demonstrates the colocalization of PLP-EGFP and LysoTracker within $100 \mathrm{~nm}$ of the plasma membrane in Oli-neu cells in coculture with neurons. (f and g) Exocytic fusion of vesicles was visualized by time-lapse TIRFM of Oli-neu cells in coculture with neurons. (f) The vesicles that lost their fluorescence during the time of observation fell into two groups. An example of vesicles moving in and out of the evanescent field without fusing is shown in the top panels. Vesicles fusing with the plasma membrane are shown in the bottom panels (indicated by arrows) and in $\mathrm{g}$. Increase in brightness, lateral spread, and disappearance of vesicular fluorescence indicates fusions. (g) The fluorescence intensity changes (in arbitrary units) were determined in a small circle enclosing the vesicle (closed symbol) and in a concentric ring around the circle (open symbol). Other examples of fusions are shown as a flash of fluorescence (LysoTracker

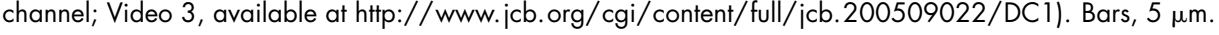




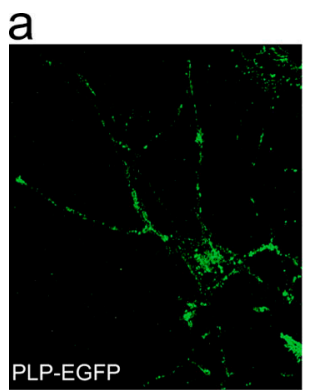

b

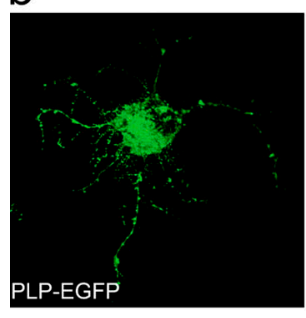

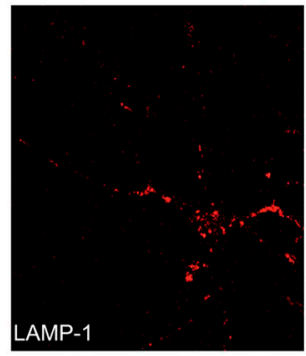
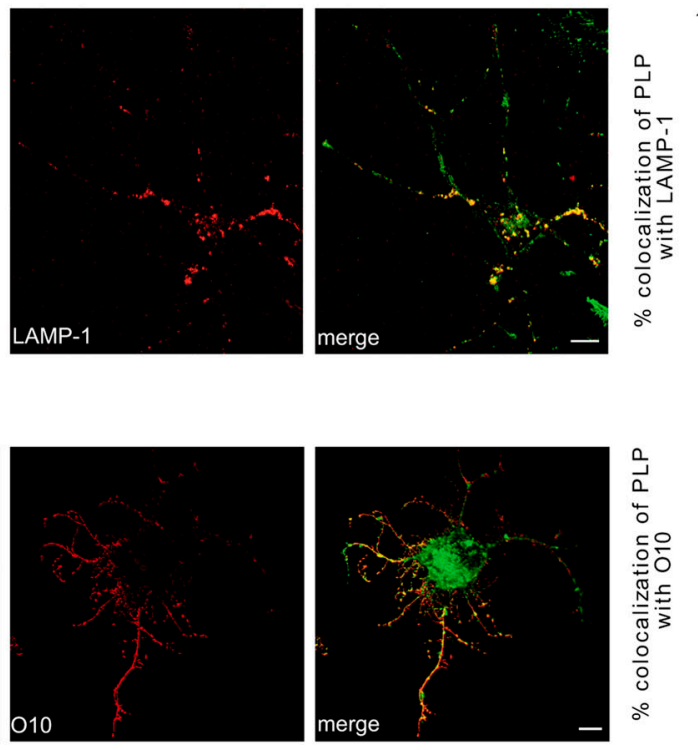
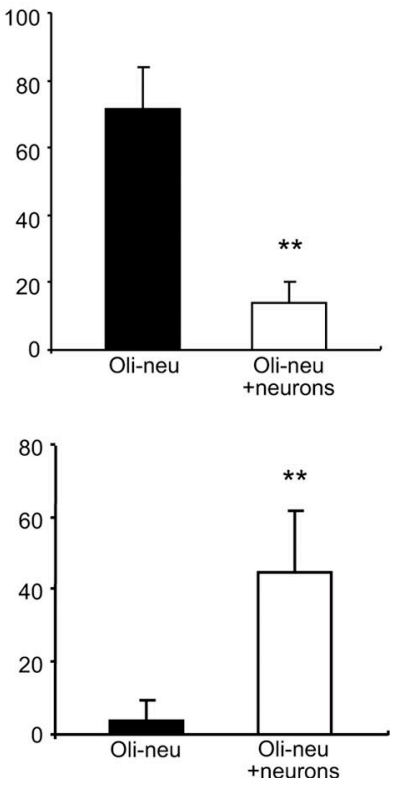

Figure 6. A soluble factor is sufficient for redistributing PLP from LEs/Ls to the cell surface. Oli-neu cells expressing PLP-EGFP were added to a neuronal culture on a separate coverslip to prevent cell contact allowing diffusible factors to reach the cells. After $1 \mathrm{~d}$, cells were stained for Lamp-1 (a) or for surface PLP with $\mathrm{O} 10 \mathrm{mAb}(\mathrm{b})$ on living cells at $4^{\circ} \mathrm{C}$. Quantitative analysis of the colocalization of PLP-EGFP with Lamp-1 and O10 are shown. Error bars represent $\mathrm{SD}\left(n>60\right.$ cells). ${ }^{*}, \mathrm{P}<0.001 ; t$ test. Bars, $5 \mu \mathrm{m}$.

LysoTracker in PLP-EGFP-expressing Oli-neu cells. There was an almost complete colocalization of PLP-EGFP and LysoTracker (Fig. 5 a) similar to the observation in OLN-93 cells. Analysis of the dynamics of PLP-EGFP/LysoTracker vesicles in Oli-neu cells revealed that most vesicles were clustered perinuclearly and did not show any significant movement (Fig. 5 a and Video 1, available at http://www.jcb.org/cgi/content/full/jcb. 200509022/DC1). Next, we investigated whether the movement of the vesicles changes as a result of the presence of neurons. When live cell imaging experiments were performed shortly (6-12 h) after the addition of Oli-neu cells to neuronal cultures, an extensive colocalization of PLP-EGFP and LysoTracker (Fig. 5 b) was still observed. However, the movement of these vesicles was markedly increased. Two pools of vesicles could be distinguished: a perinuclear, immobile pool and a peripheral pool of highly mobile vesicles (Fig. 5 b and Video 2). Both pools of vesicles colocalized with Lamp-1. Quantitative analysis revealed that $\sim 29 \%$ of the vesicles were mobile and exhibited a mean speed of $\sim 0.56 \mu \mathrm{m} / \mathrm{s}$ (Fig. $5 \mathrm{c}$ ). The pool of perinuclear vesicles not only decreased in size (Fig. 2), but the individual vesicles also became smaller with increasing time in coculture $(\sim 33 \%$ decrease after $16 \mathrm{~h}$ of coculture; reduction from $0.96 \pm 0.2 \mu \mathrm{m}$ to $0.63 \pm 0.19 \mu \mathrm{m} ; n=96$ ). In several cases, PLP-EGFP and LysoTracker-filled vesicles emanated from larger perinuclear vesicles and moved radially toward the plasma membrane at the cell periphery (Fig. $5 \mathrm{~d}$ ).

To analyze the behavior of the peripheral vesicle pool, we used total internal reflection fluorescence microscopy (TIRFM). TIRFM allows the selective illumination of a region within a 70-120-nm distance of the plasma membrane as the excitatory evanescent field decays exponentially from the interface of the cell membrane with the coverslip. We observed PLP-EGFP and
LysoTracker-containing vesicles within the 100-nm vicinity of the plasma membrane in living Oli-neu cells that were cocultured with neurons (Fig. 5e). In contrast, no PLP-EGFP and LysoTrackercontaining vesicles were observed in the proximity of the plasma membrane when cells were cultured without neurons.

To determine whether the acidic vesicles fuse with the plasma membrane, we used time-lapse TIRFM imaging. The cells we studied contained $6 \pm 2.4$ vesicles per $100-\mu \mathrm{m}^{2}$ area of the plasma membrane. The vesicles that lost their fluorescence during the time of observation fell into two groups. We found vesicles moving in and out of the evanescent field without fusing and vesicles fusing with the plasma membrane (Fig. $5 \mathrm{f}$, top). Fusion was defined by the loss of vesicular fluorescence and the concurrent lateral spread of the released dye into the medium (Fig. 5 g). We detected one to two fusion events/minute per cell at the plasma membrane. When Oli-neu cells were cultured without neurons, we did not observe any fusions in agreement with the absence of PLP-EGFP and LysoTracker-containing vesicles at the plasma membrane.

\section{A cAMP-dependent neuronal signal regulates the trafficking of PLP}

Because the redistribution of PLP from LEs/Ls to the surface of the plasma membrane was only observed in Oli-neu cells grown in the presence of neurons, neuronal signals are likely to activate this pathway. We wanted to determine whether this neuronal signaling is transferred as a soluble factor or is a consequence of direct cell-to-cell contact. Oli-neu cells were either directly added on top of a neuronal culture or placed on a separate coverslip to prevent cell contact, allowing diffusible factors to reach the cells. We found that diffusible factors were sufficient to redistribute PLP from LEs/Ls to the surface of the cell (Fig. 6). 

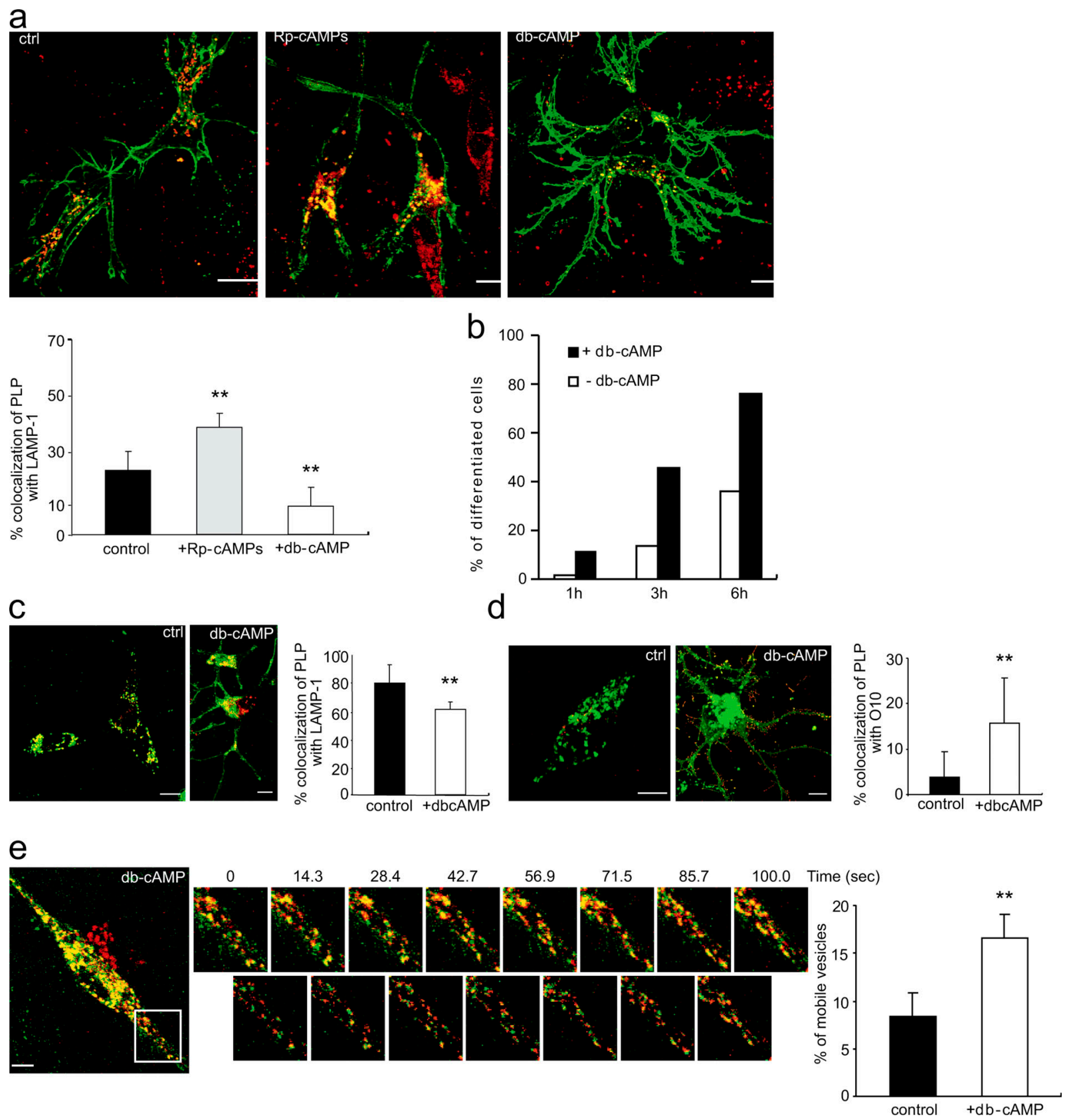

Figure 7. A cAMP-dependent neuronal signal regulates the release of PLP from LEs/Ls in Oli-neu cells. (a) Oli-neu cells were cocultured with neurons for $1 \mathrm{~d}$ in the presence or absence of $100 \mu \mathrm{M}$ Rp-cAMPs or $1 \mathrm{mM}$ db-cAMP. Quantitative analysis of the colocalization of PLP-EGFP with Lamp-1 is shown as the mean \pm SD of $>30$ cells. (b) Time-course experiments demonstrate that treatment of Oli-neu cells in the presence of neurons with $1 \mathrm{mM}$ $\mathrm{db}$-cAMP accelerates the removal of PLP-EGFP from Lamp-1-positive compartments. The quantitative analysis of one representative experiment (out of three independent experiments) is shown. Analysis was performed by classifying cells into two categories according to the extent of the colocalization of PLP-EGFP with Lamp-1. (c and d) Oli-neu cells were grown without neurons and treated with $1 \mathrm{mM}$ db-cAMP for $1 \mathrm{~d}$ and stained for Lamp-1 (c) or for surface-PLP with $\mathrm{O} 10 \mathrm{mAb}$ (d) on living cells at $4^{\circ} \mathrm{C}$. Quantitative analysis of the colocalization of PLP-EGFP with Lamp-1 and O10 are shown. Error bars represent SD ( $n=30$ cells). (e) Oli-neu cells grown without neurons were treated for $1 \mathrm{~d}$ with $1 \mathrm{mM} \mathrm{db}$-cAMP and imaged as in Fig. $5 \mathrm{a}$. An image sequence of the boxed area is shown. The images in the bottom panels represent subtractions of the consecutive frames in the top panels. The analysis demonstrates a significant increase in the mobility of PLP and LysoTracker-containing vesicles by treatment with db-cAMP as compared with the control condition. ${ }^{*}, \mathrm{P}<0.001 ; t$ test. Bars, $5 \mu \mathrm{m}$.

To analyze the signals involved, we treated Oli-neu cells cultured in the presence of neurons with various pharmacological kinase inhibitors. Colocalization of PLP-EGFP and Lamp-1 increased markedly when cocultures were treated for $1 \mathrm{~d}$ with Rp-cAMPs to inhibit protein kinase A (Fig. 7 A). In contrast, treatment of cocultures with db-cAMP, a protein kinase A agonist, promoted the localization of PLP to the plasma membrane (Fig. 7 A). Quantitative analysis of timecourse experiments showed that db-cAMP accelerated the redistribution of PLP to the surface of the cell (Fig. 7 B). To test whether similar effects were observed in Oli-neu cells cultured without neurons, we treated Oli-neu cells for $1 \mathrm{~d}$ with db-cAMP 
and quantified the amount of PLP-EGFP in LEs/Ls and on the surface of the cell. In cells treated with db-cAMP, more PLP was found on the surface of the cell, whereas, at the same time, the fraction within LEs/Ls decreased (Fig. 7, C and D). Furthermore, a peripheral pool of highly mobile PLP-EGFP/ LysoTracker-containing vesicles was observed by live cell imaging (Fig. 7 E). These data suggest that cAMP-dependent signaling is part of the developmental switch that is triggered by neurons, leading to the redistribution of PLP from LEs/Ls to the surface of the plasma membrane.

\section{iscussion}

Our data demonstrate that the trafficking of PLP in oligodendrocytes is under neuronal control. PLP is initially targeted to LEs/Ls by using a cholesterol-dependent and clathrin-independent endocytosis pathway. The situation changes dramatically upon receiving maturation signals from neurons. PLP is then redistributed from LEs/Ls to the plasma membrane. We provide evidence that the developmental regulation of PLP localization occurs by the down-regulation of endocytosis and by the transport from LEs/Ls to the cell surface.

The regulation of the transport of PLP is strikingly similar to the trafficking of MHC-II in dendritic cells (Mellman and Steinman, 2001). Immature dendritic cells have a high rate of endocytosis and target MHC-II to lysosomes (Pierre et al., 1997). After exposure to inflammatory mediators, the endocytosis of MHC-II is reduced, and the transport of MHC-II from lysosomes to the cell surface is triggered (Kleijmeer et al., 2001; Boes et al., 2002; Chow et al., 2002). The transport pathway of PLP from LEs/Ls can also be related to the release of secretory lysosomes from hematopoietic cells. However, unlike the classic secretory lysosomes that are specialized to release luminal content, oligodendrocytes mainly transport membrane, and this may occur without significant extracellular release of lysosomal content, as is the case for dendritic cells (Kleijmeer et al., 2001; Trombetta et al., 2003). For oligodendrocytes, LE/L compartments may be particularly useful as storage compartments, as they are able to harbor large amounts of membrane in a multilamellar and multivesicular fashion for myelin biogenesis. In most cells, however, the majority of molecules that localizes to internal vesicles of the endosomal system are destined for lysosomal degradation. This raises the question of how PLP survives in an environment where protein degradation usually occurs. One possibility is that immature oligodendrocytes have specialized LEs/Ls with low proteolytic capacity. Our unpublished observation that the vesicular stomatitis virus glycoprotein accumulates undegraded in LEs/Ls of Oli-neu cells supports this notion. In dendritic cells, for example, lysosomal proteolysis is regulated in a developmentally linked fashion (Trombetta et al., 2003; Delamarre et al., 2005). Another possibility is that PLP is poorly degradable and, therefore, accumulates within LEs/Ls. A second issue is how PLP escapes from a compartment associated with the limited capacity for membrane recycling. Previous work has provided evidence that not all intralumenal membranes of LEs/Ls are destined for lysosomal degradation. It has been suggested that some vesicles may undergo back fusion with the limiting membrane, and, in some instances, this membrane is sorted via tubulovesicles to the plasma membrane (Kleijmeer et al., 2001; Boes et al., 2002; Chow et al., 2002; Abrami et al., 2004; Le Blanc et al., 2005). Whether PLP is sorted by back fusion and tubules to the surface of oligodendrocytes are issues that have to be addressed in future studies. It is important to note that the accumulation of PLP at the surface of the plasma membrane after the receipt of maturation signals most likely reflects the contribution of multiple factors. Our finding that the endocytosis of PLP is reduced after receiving signals from neuronal cells suggests that the regulation of endocytosis may play an essential role in this process. It will be interesting to elucidate the molecular mechanisms of how neurons control the rate of endocytosis in oligodendrocytes. One attractive possibility is that the endocytic activity is controlled by the RhoA GTPase.

Importantly, not all myelin components were found to be internalized into LEs/Ls. Although PLP and cholesterol resided in LEs/Ls, myelin basic protein and galactosylceramide were mainly found in or at the plasma membrane (unpublished data). The differential compartmentalization of myelin components before the onset of myelination might be a mechanism to prevent premature and inappropriate assembly. Our results suggest that an external soluble factor regulates myelin membrane assembly by controlling the trafficking of PLP to and from the surface of the cell. Among the many potential candidates are soluble mediators such as neurotrophins, neuregulin, or adenosine that can now be tested with the described experimental system (Barres and Raff, 1999; Fields and StevensGraham, 2002).

Together, our findings reveal an unexpected and novel role of LEs/Ls in oligodendrocytes. It provides a striking example of how cell-to-cell communication regulates trafficking to and from a cellular compartment to guide the development of a multicellular tissue. The proposed role of LEs/Ls in myelin biogenesis may help to explain the cellular mechanisms of dysmyelination that is observed in many lysosomal storage diseases.

\section{Materials and methods}

\section{Antibodies, plasmids, and other reagents}

The mutant and wild-type cDNAs of GFP-Eps 15 and GFP-dynamin-II were provided by A. Benmerah (Institut Pasteur, Paris, France) and S. Schmid (Scripps Research Institute, La Jolla, CA), respectively. The following primary antibodies were used: myelin basic protein (monoclonal lgG; Sternberger, Inc.), PLP (polyclonal, P6; Linington and Waehneldt, 1990), O10 (monoclonal mouse lgM), $\beta$ Ill-tubulin (Promega), neurofilament (monoclonal lgM; Qbiogene), $\tau$ (polyclonal; DakoCytomation), myc tag (monoclonal lgG; Cell Signaling), clathrin heavy chain (monoclonal lgG; BD Transduction Laboratories), GFP (Synaptic Systems GmbH), caveolin-1 (monoclonal lgM; BD Biosciences), and Lamp-1 (CD107a, rat monoclonal; $\mathrm{BD}$ Biosciences). Secondary antibodies were obtained from Dianova and GE Healthcare.

\section{Cell culture, cloning, and transfections}

Primary cultures of mouse oligodendrocytes were prepared as described previously (Simons et al., 2000). After shaking, cells were plated in MEM containing B27 supplement, 1\% horse serum, L-thyroxine, tri-iodothyronine, glucose, glutamine, gentamycine, pyruvate, and bicarbonate on poly-L-lysine-coated dishes or glass coverslips. Cocultures of neurons and oligodendrocytes were produced by preparing mixed brain cultures from 16-d-old fetal mice that were cultivated for $2 \mathrm{wk}$, to which the primary oligodendrocytes or Oli-neu cells were added. The mixed brain cultures were 
prepared at a density of $\sim 50,000$ cells $/ \mathrm{cm}^{2}$. Cocultures without direct neuron-glia contact were prepared by growing neuronal cultures on glass coverslips, which were placed upside down on a metal ring positioned in a culture dish. Oligodendrocytes were added on an additional coverslip facing upwards. The oligodendroglial precursor cell line, Oli-neu (provided by J. Trotter, University of Mainz, Mainz, Germany), and OLN-93 cells (provided by C. Richter-Landsberg, University of Oldenburg, Oldenburg, Germany) were cultured as described previously (Jung et al., 1995; RichterLandsberg and Heinrich, 1996). Transient transfections were performed using FuGENE transfection reagent (Roche) according to the manufacturer's protocol. PLP-EGFP was generated by fusing EGFP to the $\mathrm{COOH}$ terminus of PLP by gene fusion PCR. The fusion product was cloned into pEGFPNI vector using the EcoRI-Notl site. Stable cell lines were obtained by the cotransfection of PLP-EGFPNI and pMSCV-hygro (CLONTECH Laboratories, Inc.) followed by the selection of clones by incubation with hygromycin.

\section{Immunofluorescence and endocytosis assays}

Immunofluorescence and immunohistochemistry were performed as described previously (Simons et al., 2002). For assaying endocytosis, living cells were incubated with 010 antibody in medium for $30 \mathrm{~min}$ at $4^{\circ} \mathrm{C}$, washed, and incubated in medium at $37^{\circ} \mathrm{C}$ for $60 \mathrm{~min}$. The antibody remaining on the surface was removed under low $\mathrm{pH}$ conditions in $0.2 \mathrm{M}$ glycine and $0.5 \mathrm{M} \mathrm{NaCl}, \mathrm{pH} 4.5$, for $30 \mathrm{~min}$ at $4^{\circ} \mathrm{C}$. Cells were washed three times in PBS, fixed, and stained by immunofluorescence. Additionally, OLN-93 cells were transiently transfected with PLP and added on a neuronal culture or left alone. Cells were incubated at $4^{\circ} \mathrm{C}$ for $30 \mathrm{~min}$ with $\mathrm{O} 10$ antibody in binding medium consisting of HBSS and $10 \mathrm{mM}$ Hepes supplemented with $0.2 \% \mathrm{BSA} 16 \mathrm{~h}$ after transfection. After washing, $\mathrm{O} 10$ internalization was allowed to continue for 0,45 , and $90 \mathrm{~min}$. 010 antibody remaining at the cell surface was detected with $5-20 \mu \mathrm{Ci} / \mu \mathrm{g}$ [125|]-labeled mouse secondary antibody in binding medium for $30 \mathrm{~min}$ at $4^{\circ} \mathrm{C}$. Next, the cells were washed five times, lysed in $0.2 \mathrm{M} \mathrm{NaOH}$, and the amount of radioactivity was determined by $\gamma$ counting.

\section{Microscopy and analysis}

Fluorescence images were acquired on a microscope (DMRXA; Leica) or a confocal microscope (LSM 510; Carl Zeiss Microlmaging, Inc.) with a $63 \times$ oil plan-Apochromat objective (NA 1.4; Carl Zeiss Microlmaging, Inc.). For live cell imaging, coverslips containing the cells were mounted in a live cell imaging chamber and observed in low fluorescence imaging medium (HBSS, $10 \mathrm{mM}$ Hepes, and $1 \%$ horse serum, $\mathrm{pH} \mathrm{7.4)} \mathrm{at} 37^{\circ} \mathrm{C}$. Temperature was controlled by means of a digital system (Tempcontrol 37-2; PeCon) or a custom-built perfusion system. Time-lapse imaging was performed on a confocal laser scanning microscope (LSM 510; Carl Zeiss Microlmaging, Inc.). Images were acquired at $\sim 15$-s intervals for the indicated time periods using sequential line excitation at 488 and $543 \mathrm{~nm}$ and appropriate band pass emission filters.

Image processing and analysis were performed using Meta Imaging Series 6.1 software (Universal Imaging Corp.). Quantification of colocalization was performed with the colocalization module of the software. Vesicle movement was analyzed by subtracting from each image in a time stack preceding its image. The different image stack thus generated was used to identify vesicle motility events. The velocity of individual vesicles was determined using the Manual Tracking plug-in for ImageJ software (National Institutes of Health). Statistical differences were determined with a $t$ test. TIRFM was performed on a custom-built prism-based evanescent field microscope using an HCX Apo L 63× water immersion objective (NA 0.90; Leica; Oheim et al., 1998). Evanescent field excitation was obtained by focusing 488- and 568-nm laser light onto a hemicylindrical prism at 68 and $71^{\circ}$ incidence angles, respectively, leading to a field depth of $\sim 80-100 \mathrm{~nm}$. Images were acquired with a back-illuminated 16-bit CCD camera (Cascade 512B; Roper Scientific) with on-chip charge multiplication. Each pixel corresponded to $0.25 \mu \mathrm{m}$ in the specimen plane. For analysis of individual fusion events, a small circle was positioned on the vesicular fluorescence, a concentric ring was placed around the circle, and fluorescence intensity was plotted against time. Fusion events were identified by the increase of fluorescence in the central region that spread into the surrounding annulus followed by a sudden decline (Schmoranzer et al., 2000; Zenisek et al., 2000; Becherer et al., 2003; Bezzi et al., 2004). Immuno-EM was performed as described previously (Wenzel et al., 2005).

\section{Metabolic labeling and immunoprecipitations}

For metabolic labeling, cells were pulsed with $265 \mu \mathrm{Ci}\left[{ }^{35} \mathrm{~S}\right]$ methionine (1 $\mathrm{Ci}=37 \mathrm{GBq}$; GE Healthcare) in methionine and cysteine-free DME for $18 \mathrm{~h}$, and chase was performed for 0 or $48 \mathrm{~h}$. Immunoprecipitation was performed as described previously (Simons et al., 2000). Autoradiographs were scanned and quantified with Scionlmage software (Scion Corp.). Values are shown as means \pm SD. Statistical differences were determined with a $t$ test.

\section{Online supplemental material}

Fig. S1 shows that EGFP-tagged PLP is sorted to myelin. Fig. S2 shows the absence of PLP colocalization with clathrin heavy chain or caveolin-1. Video 1 shows the movement of LEs/Ls in Oli-neu cells, whereas Video 2 shows this in Oli-neu cells cultured with neurons. Video 3 shows the fusion of vesicles with the plasma membrane. Online supplemental material is available at http://www.jcb.org/cgi/content/full/jcb.200509022/DCl.

We are grateful to $G$. Schulz for excellent technical assistance. We thank C. Richter-Landsberg and J. Trotter for providing reagents, J. Landgrebe for help with iodine-125 experiments, and W. Stühmer for support.

This work was supported by the Deutsche Forschungsgemeinschaft (SFB 523 to M. Simons and K.A. Nave and SFB 406 to G. Bunt).

Submitted: 6 September 2005

Accepted: 26 January 2006

\section{References}

Abrami, L., M. Lindsay, R.G. Parton, S.H. Leppla, and F.G. van der Goot. 2004. Membrane insertion of anthrax protective antigen and cytoplasmic delivery of lethal factor occur at different stages of the endocytic pathway. J. Cell Biol. 166:645-651.

Barres, B.A., and M.C. Raff. 1999. Axonal control of oligodendrocyte development. J. Cell Biol. 147:1123-1128.

Baumann, N., and D. Pham-Dinh. 2001. Biology of oligodendrocyte and myelin in the mammalian central nervous system. Physiol. Rev. 81:871-927.

Becherer, U., T. Moser, W. Stuhmer, and M. Oheim. 2003. Calcium regulates exocytosis at the level of single vesicles. Nat. Neurosci. 6:846-853.

Benmerah, A., M. Bayrou, N. Cerf-Bensussan, and A. Dautry-Varsat. 1999. Inhibition of clathrin-coated pit assembly by an Eps15 mutant. J. Cell Sci. 112:1303-1311.

Bezzi, P., V. Gundersen, J.L. Galbete, G. Seifert, C. Steinhauser, E. Pilati, and A. Volterra. 2004. Astrocytes contain a vesicular compartment that is competent for regulated exocytosis of glutamate. Nat. Neurosci. 7:613-620.

Blott, E.J., and G.M. Griffiths. 2002. Secretory lysosomes. Nat. Rev. Mol. Cell Biol. 3:122-131.

Boes, M., J. Cerny, R. Massol, M. Op den Brouw, T. Kirchhausen, J. Chen, and H.L. Ploegh. 2002. T-cell engagement of dendritic cells rapidly rearranges MHC class II transport. Nature. 418:983-988.

Chow, A., D. Toomre, W. Garrett, and I. Mellman. 2002. Dendritic cell maturation triggers retrograde MHC class II transport from lysosomes to the plasma membrane. Nature. 418:988-994.

Damke, H., T. Baba, D.E. Warnock, and S.L. Schmid. 1994. Induction of mutant dynamin specifically blocks endocytic coated vesicle formation. J. Cell Biol. 127:915-934.

Damm , E.M., L. Pelkmans, J. Kartenbeck, A. Mezzacasa, T. Kurzchalia, and A. Helenius. 2005. Clathrin- and caveolin-1-independent endocytosis: entry of simian virus 40 into cells devoid of caveolae. J. Cell Biol. $168: 477-488$.

Delamarre, L., M. Pack, H. Chang, I. Mellman, and E.S. Trombetta. 2005. Differential lysosomal proteolysis in antigen-presenting cells determines antigen fate. Science. 307:1630-1634.

Fields, R.D., and B. Stevens-Graham. 2002. New insights into neuron-glia communication. Science. 298:556-562.

Jung, M., E. Kramer, M. Grzenkowski, K. Tang, W. Blakemore, A. Aguzzi, K. Khazaie, K. Chlichlia, G. von Blankenfeld, H. Kettenmann, et al. 1995. Lines of murine oligodendroglial precursor cells immortalized by an activated neu tyrosine kinase show distinct degrees of interaction with axons in vitro and in vivo. Eur. J. Neurosci. 7:1245-1265.

Jung, M., I. Sommer, M. Schachner, and K.A. Nave. 1996. Monoclonal antibody O10 defines a conformationally sensitive cell-surface epitope of proteolipid protein (PLP): evidence that PLP misfolding underlies dysmyelination in mutant mice. J. Neurosci. 16:7920-7929.

Kirkham, M., A. Fujita, R. Chadda, S.J. Nixon, T.V. Kurzchalia, D.K. Sharma, R.E. Pagano, J.F. Hancock, S. Mayor, and R.G. Parton. 2005. Ultrastructural identification of uncoated caveolin-independent early endocytic vehicles. J. Cell Biol. 168:465-476.

Kleijmeer, M., G. Ramm, D. Schuurhuis, J. Griffith, M. Rescigno, P. RicciardiCastagnoli, A.Y. Rudensky, F. Ossendorp, C.J. Melief, W. Stoorvogel, and H.J. Geuze. 2001. Reorganization of multivesicular bodies regulates 
MHC class II antigen presentation by dendritic cells. J. Cell Biol. 155:53-63.

Kramer, E.M., A. Schardt, and K.A. Nave. 2001. Membrane traffic in myelinating oligodendrocytes. Microsc. Res. Tech. 52:656-671.

Lamaze, C., A. Dujeancourt, T. Baba, C.G. Lo, A. Benmerah, and A. DautryVarsat. 2001. Interleukin 2 receptors and detergent-resistant membrane domains define a clathrin-independent endocytic pathway. Mol. Cell. 7:661-671.

Le Blanc, I., P.P. Luyet, V. Pons, C. Ferguson, N. Emans, A. Petiot, N. Mayran, N. Demaurex, J. Faure, R. Sadoul, et al. 2005. Endosome-to-cytosol transport of viral nucleocapsids. Nat. Cell Biol. 7:653-664.

Linington, C., and T.V. Waehneldt. 1990. Conservation of the carboxyl terminal epitope of myelin proteolipid protein in the tetrapods and lobe-finned fish. J. Neurochem. 54:1354-1359.

Mellman, I., and R.M. Steinman. 2001. Dendritic cells: specialized and regulated antigen processing machines. Cell. 106:255-258.

Oheim, M., D. Loerke, W. Stuhmer, and R.H. Chow. 1998. The last few milliseconds in the life of a secretory granule. Docking, dynamics and fusion visualized by total internal reflection fluorescence microscopy (TIRFM). Eur. Biophys. J. 27:83-98.

Parton, R.G., and A.A. Richards. 2003. Lipid rafts and caveolae as portals for endocytosis: new insights and common mechanisms. Traffic. 4:724-738.

Pelkmans, L., J. Kartenbeck, and A. Helenius. 2001. Caveolar endocytosis of simian virus 40 reveals a new two-step vesicular-transport pathway to the ER. Nat. Cell Biol. 3:473-483.

Pierre, P., S.J. Turley, E. Gatti, M. Hull, J. Meltzer, A. Mirza, K. Inaba, R.M. Steinman, and I. Mellman. 1997. Developmental regulation of MHC class II transport in mouse dendritic cells. Nature. 388:787-792.

Richter-Landsberg, C., and M. Heinrich. 1996. OLN-93: a new permanent oligodendroglia cell line derived from primary rat brain glial cultures. J. Neurosci. Res. 45:161-173.

Sabharanjak, S., P. Sharma, R.G. Parton, and S. Mayor. 2002. GPI-anchored proteins are delivered to recycling endosomes via a distinct cdc42-regulated, clathrin-independent pinocytic pathway. Dev. Cell. 2:411-423.

Schmoranzer, J., M. Goulian, D. Axelrod, and S.M. Simon. 2000. Imaging constitutive exocytosis with total internal reflection fluorescence microscopy. J. Cell Biol. 149:23-32.

Schneider, A., H. Lander, G. Schulz, H. Wolburg, K.A. Nave, J.B. Schulz, and M. Simons. 2005. Palmitoylation is a sorting determinant for transport to the myelin membrane. J. Cell Sci. 118:2415-2423.

Simons, M., E.M. Kramer, C. Thiele, W. Stoffel, and J. Trotter. 2000. Assembly of myelin by association of proteolipid protein with cholesterol- and galactosylceramide-rich membrane domains. J. Cell Biol. 151:143-154.

Simons, M., E.M. Kramer, P. Macchi, S. Rathke-Hartlieb, J. Trotter, K.A. Nave, and J.B. Schulz. 2002. Overexpression of the myelin proteolipid protein leads to accumulation of cholesterol and proteolipid protein in endosomes/lysosomes: implications for Pelizaeus-Merzbacher disease. J. Cell Biol. 157:327-336.

Trombetta, E.S., M. Ebersold, W. Garrett, M. Pypaert, and I. Mellman. 2003. Activation of lysosomal function during dendritic cell maturation. Science. 299:1400-1403.

Weimbs, T., and W. Stoffel. 1992. Proteolipid protein (PLP) of CNS myelin: positions of free, disulfide-bonded, and fatty acid thioester-linked cysteine residues and implications for the membrane topology of PLP. Biochemistry. 31:12289-12296.

Wenzel, D., G. Schauermann, A. von Lupke, and G. Hinz. 2005. The cargo in vacuolar storage protein transport vesicles is stratified. Traffic. 6:45-55.

Zenisek, D., J.A. Steyer, and W. Almers. 2000. Transport, capture and exocytosis of single synaptic vesicles at active zones. Nature. 406:849-854. 
3.2. Publication 2:

Rho regulates membrane transport in the endocytic pathway to control plasma membrane specialization in oligodendroglial cells 


\title{
Rho Regulates Membrane Transport in the Endocytic Pathway to Control Plasma Membrane Specialization in Oligodendroglial Cells
}

\author{
Angelika Kippert, ${ }^{1,2 \star}$ Katarina Trajkovic, ${ }^{1,2 \star}$ Lawrence Rajendran, ${ }^{4}$ Jonas Ries, ${ }^{3}$ and Mikael Simons ${ }^{1,2}$ \\ ${ }^{1}$ Centre for Biochemistry and Molecular Cell Biology, University of Göttingen, 37073 Göttingen, Germany, ${ }^{2}$ Max-Planck-Institute for Experimental \\ Medicine, 37075 Göttingen, Germany, ${ }^{3}$ Technical University of Dresden, 01062 Dresden, Germany, and ${ }^{4}$ Max-Planck-Institute of Molecular Cell Biology and \\ Genetics, 01307 Dresden, Germany
}

\begin{abstract}
Differentiation of oligodendrocytes is associated with dramatic changes in plasma membrane structure, culminating in the formation of myelin membrane sheaths. Previous results have provided evidence that regulation of endocytosis may represent a mechanism to control myelin membrane growth. Immature oligodendrocytes have a high rate of clathrin-independent endocytosis for the transport of membrane to late endosomes/lysosomes (LE/Ls). After maturation and receiving signals from neurons, endocytosis is reduced and transport of membrane from LE/Ls to the plasma membrane is triggered. Here, we show that changes in Rho GTPase activity are responsible for switching between these two modes of membrane transport. Strikingly, Rho inactivation did not only reduce the transport of cargo to $\mathrm{LE} / \mathrm{L}$ but also increased the dynamics of LE/L vesicles. Furthermore, we provide evidence that Rho inactivation results in the condensation of the plasma membrane in a polarized manner. In summary, our data reveal a novel role of Rho: to regulate the flow of membrane and to promote changes in cell surface structure and polarity in oligodendroglial cells. We suggest that Rho inactivation is required to trigger plasma membrane specialization in oligodendrocytes.
\end{abstract}

Key words: oligodendrocytes; neurons; myelin; Rho GTPases; endocytosis; late endosomes/lysosomes

\section{Introduction}

Differentiation of oligodendrocytes is an extreme example of cell specialization characterized by the outgrowth of an extensive amount of plasma membrane. This process leads to the generation of myelin and is associated with dramatic changes in plasma membrane structure and composition (Salzer, 2003; Sherman and Brophy, 2005). Although myelin is still continuous with the plasma membrane, its composition differs fundamentally from that of the membrane surrounding the cell body (Taylor et al., 2004; Debruin and Harauz, 2007; Gielen et al., 2006). Myelin has an exceptionally high content of lipids (70\% of dry weight), in particular galactosylceramide and cholesterol, and contains a small number of different proteins, which are in most cases mainly found in myelin. The major myelin proteins in compact myelin, the proteolipid proteins (PLP/DM20), are highly hydrophobic, cholesterol-interacting proteins (Milner et al., 1985; Simons et al., 2000). Intuitively, one would assume that the synthesis of myelin membrane proteins and lipids is turned on by

Received Nov. 13, 2006; revised Feb. 14, 2007; accepted Feb. 20, 2007.

This work was supported by the Deutsche Forschungsgemeinschaft (SFB 523). We thank G. Schulz for technical assistance and E. Papoucheva for help with the FRET analysis. We are grateful to M. Matsuda, A. Benmerah, S. Schmid, S. Gutkind, and G. Superti-Furga for providing reagents.

${ }^{*}$ A.K. and K.T. contributed equally to this work.

Correspondence should be addressed to Dr. Mikael Simons, Centre for Biochemistry and Molecular Cell Biology, University of Göttingen, Humboldallee 23, 37073 Göttingen, Germany. E-mail: msimons@gwdg.de.

D0I:10.1523/JNEUROSCI.4926-06.2007

Copyright $\odot 2007$ Society for Neuroscience $\quad$ 0270-6474/07/273560-11\$15.00/0 neuronal signals, because they are required at the time oligodendrocytes begin wrapping their membrane around axons. Surprisingly, the gene expression of the major myelin components is initiated and also continues at a high rate in primary cultures of oligodendrocytes in the absence of neurons (Mirsky et al., 1980; Dubois-Dalcq et al., 1986; Dugas et al., 2006). However, because the ensheathment of axons must occur at the appropriate time of neuronal development, reciprocal communication between neurons and oligodendrocytes plays an important role in the coordination of myelin membrane growth (Barres and Raff, 1999; Edgar et al., 2004; Schafer and Rasband, 2006; Simons and Trajkovic, 2006). Recent work has provided evidence that myelin membrane trafficking is under the control of neuronal signals (Trajkovic et al., 2006). In the absence of neurons, PLP is internalized and stored in late endosomes/lysosomes (LE/Ls). After receiving an unknown diffusible neuronal factor, endocytosis of PLP is downregulated and transport of PLP from LE/L to the plasma membrane is triggered. The aim of the present work was to investigate the molecular mechanism of how oligodendrocytes switch between these two modes of membrane transport. We found that changes in Rho GTPase activity play a critical role in this process by regulating the flow of membrane through the endosomal system.

\section{Materials and Methods}

Antibodies and plasmids. The following plasmids were used: mutant and wild-type cDNAs of green fluorescent protein (GFP)-Eps15 (A. Benmerah, Institut Pasteur, Paris, France), mutant and wild-type GFP- 
dynaminII (S. Schmid, Scripps Research Institute, La Jolla, CA), RaichuRhoA-1237X (M. Matsuda, Kyoto University, Kyoto, Japan) (Yoshizaki et al., 2003), wild-type and mutant RhoA, RhoB, and RhoC (obtained from the Unité Mixte de Recherche cDNA Resource Centre), wild-type and mutant c-Src (G. Superti-Furga, EMBL, Heidelberg, Germany; S. Gutkind, National Institutes of Health, Bethesda, MD) (Sandilands et al., 2004); the N-terminal 20 amino acids of neuromodulin (which contain a consensus sequence for dual palmitoylation) fused to GFP (membranetargeted GFP) (Schneider et al., 2005). The following primary antibodies were used: hemagglutinin (HA) (16B12, mouse monoclonal; Covance, Berkley, CA), HA (rabbit polyclonal IgG; Abcam, Cambridge, UK), GFP (polyclonal rabbit; Abcam), GM130 (mouse monoclonal IgG1; BD Biosciences, Franklin Lakes, NJ), Lamp-1 (CD 107a, rat monoclonal; BD Biosciences), myc (rabbit polyclonal IgG; Upstate Biotechnology, Temecula, CA), myc (monoclonal IgG; Cell Signaling Technology, Denver, CO), O10 (monoclonal mouse IgM) (Jung et al., 1996), RhoA (26C4, mouse monoclonal; Santa Cruz Biotechnology, Santa Cruz, CA), and Src (36D10, rabbit monoclonal IgG; Cell Signaling Technology). Secondary antibodies were purchased from Dianova (Hamburg, Germany).

Cell culture, cloning, and transfections. Primary cultures of mouse oligodendrocytes were prepared as described previously (Simons et al., 2000). After shaking, cells were plated in MEM containing B27 supplement, 1\% horse serum (HS), L-thyroxine, tri-iodo-thyronine, glucose, glutamine, gentamycine, pyruvate, and bicarbonate on poly-L-lysinecoated dishes or glass coverslips. The oligodendroglial precursor cell line, Oli-neu, was cultured as described previously (Jung et al., 1995). For all experiments, Oli-neu cells with a low passage number were used. Primary cultures enriched in neurons were obtained by preparing a mixed brain culture from 16-d-old fetal mice. The cells were plated at a density of $\sim 200000$ cells $/ \mathrm{cm}^{2}$ and grown for 2 weeks in Sato-B27/1\% HS. The culture was enriched in neurons but contained some astrocytes. Conditioned neuronal medium was obtained from these cultures, collected after culturing for 2 weeks, centrifuged for $5 \mathrm{~min}$ at $4000 \times \mathrm{g}$, and used directly.

Primary cultures of astrocytes were prepared from a mixed brain culture from 16-d-old fetal mice. The cells were plated on uncoated cell culture dishes and cultured in DMEM/10\% HS.

Cells were trypsinized and transferred to a new dish to remove the neurons. Cultures that were highly enriched in astrocytes were grown for 2 weeks in Sato-B27/1\% HS.

Transient transfections were performed using FuGENE transfection reagent according to the manufacturer protocol. Immunofluorescence was performed as described previously (Trajkovic et al., 2006). For delivery of recombinant C3 transferase (Cytoskeleton, Denver, CO), Chariot reagent (Active Motif, Carlsbad, CA) was used according to the manufacturer protocol.

Wild-type, dominant-negative, and constitutively active RhoBenhanced GFP (EGFP) and RhoC-EGFP fusion products were generated from the respective HA-tagged Rho constructs by cloning them into the pEGFP-C1 vector (Clontech, Mountain View, CA) using the ApaI-KpnI site.

Immunofluorescence and endocytosis assays. Endocytosis was assessed by incubating Oli-neu cells stably expressing PLP-EGFP cells for $30 \mathrm{~min}$ with Transferrin-Rhodamine (Tf) (Invitrogen, Carlsbad, CA) or Dextran-Rhodamine 10K (Dextran) (Invitrogen). Cells were subsequently washed and fixed, followed by microscopy analysis. For assaying endocytosis of glycosylphosphatidylinositol (GPI)-cyan fluorescent protein (CFP), living cells were incubated with anti-GFP antibody in medium for $30 \mathrm{~min}$ at $4^{\circ} \mathrm{C}$, washed, incubated in medium at $37^{\circ} \mathrm{C}$ for 30 $\mathrm{min}$, and fixed. Fixed, nonpermeabilized cells were labeled with Cy5conjugated secondary antibodies to visualize GPI-CFP at the cell surface and then permeabilized and labeled with Cy3-conjugated secondary antibodies to resolve internalized protein. To analyze endocytosis of $\mathrm{ChxB}$, ChxB conjugated to FITC was bound to the cell surface at $4^{\circ} \mathrm{C}$, and the cells were warmed for $40 \mathrm{~min}$ to allow transport to the Golgi complex. Using mask overlay image analysis, we quantified the internalization of FITC-ChxB (Sigma-Aldrich, Deisenhofen, Germany) to the GM130positive Golgi apparatus. Laurdan (6-dodecanoyl-2-dimethylaminonaphtalene; Invitrogen) labeling was performed in live cultures ( $5 \mu \mathrm{M}, 30$ min at $37^{\circ} \mathrm{C}$ ), followed by fixation with $4 \%$ paraformaldehyde. To deplete sphingolipids, $50 \mu \mathrm{m}$ Fumonisin B1 was added to Oli-neu cells every $24 \mathrm{~h}$ for $3 \mathrm{~d}$.

Microscopy and analysis. Fluorescence images were acquired on a Leica (Nussloch, Germany) DMRXA microscope or a Zeiss (Jena, Germany) LSM 510 confocal microscope with a $63 \times$ oil plan-apochromat objective [numerical aperture (NA), 1.4]. For live cell imaging, coverslips containing the cells were mounted in a live-cell imaging chamber and observed in low fluorescence imaging medium (HBSS; 10 mM HEPES, $1 \%$ horse serum, $\mathrm{pH} 7.4$ ) at $37^{\circ} \mathrm{C}$. Temperature was controlled by means of a Tempcontrol 37-2 digital system or a custom-built perfusion system. Time-lapse imaging was performed on a Zeiss LSM 510 confocal laserscanning microscope. Image processing and analysis was performed using Meta Imaging Series 6.1 software (Universal Imaging Corporation, West Chester, PA). Quantification of fluorescence intensities and of colocalization was performed as described previously (Trajkovic et al., 2006). Vesicle movement was analyzed by collecting images at $\sim 15 \mathrm{~s}$ intervals. Ten subsequent images were superimposed, and the first image of the time stack was subtracted from the resulting picture. The resulting subtraction is shown in green, and the first image is shown in red. Movement within the imaging period was quantified by determining the ratio of the intensity of green to red pixels [(integrated intensity of subtraction plus integrated intensity of first image)/integrated intensity of first image] (Wubbolts et al., 1996).

For flourescence resonance energy transfer (FRET) measurements, we used Raichu-RhoA-1237X (Yoshizaki et al., 2003), and FRET was detected by an increase in donor fluorescence after photobleaching of the acceptor on a confocal Leica microscope (TCS SP2 equipped with AOBS) as described previously (Fitzner et al., 2006). Image analysis of FRET data was performed using custom-written MATLAB routines.

For Laurdan experiments, we used a Zeiss LSM 510 confocal microscope with a $63 \times$ oil plan-apochromat objective (NA, 1.4; Zeiss) to excite at $364 \mathrm{~nm}$, and images were recorded simultaneously in the range of 400 to $450 \mathrm{~nm}$ and 470 to $530 \mathrm{~nm}$ for the two channels, respectively. For analysis of the Laurdan images, an average background was determined for each channel and subtracted from the intensity images, which were then converted into GP images with the generalized polarization (GP) defined by the following:

$$
\mathrm{GP}=\frac{I_{(400-450)}-I_{(470-530)}}{I_{(400-450)}+I_{(470-530)}},
$$

using software written in MATLAB (MathWorks, Natick, MA). Note that this definition of the GP depends on the setup used and allows monitoring relative changes of membrane order but cannot be compared directly to absolute values in the literature. To determine the GP values at the plasma membrane, regions of interest were selected randomly and the average GP, weighted in each pixel with the total Laurdan intensity $I(400-450)+I(470-530)$, was calculated therein (Gaus et al., 2006). Statistical differences were determined with Student's $t$ test.

Src kinase and RhoA activity measurements. Rho activity was measured by affinity precipitation of active (GTP-bound) Rho from cell lysates using a glutathione $S$-transferase (GST) fusion protein containing the Rho-binding domain of Rhotekin followed by detection with the respective antibody by immunoblotting as described previously (Ren et al., 1999).

To measure Src kinase activity, cell lysates were immunoprecipitated with anti-Src kinase antibody and subjected to a kinase assay by using an Src Assay kit (Upstate Biotechnology). The assay is based on the quantification of the phosphorylation of a synthetic Src substrate peptide (KVEKIGEGTYGVVK). The phosphorylated substrate is then separated from residual $\gamma-{ }^{32} \mathrm{P}$-ATP using p81 phosphocellulose paper, followed by the quantification with a scintillation counter.

For immunoblotting, cells were lysed and protein levels quantified using Bradford Reagent (Bio-Rad, Hercules, CA). The cell lysates were loaded on a gel, separated by SDS-PAGE, and transferred to nitrocellulose using standard protocols. 


\section{Results}

Subcellular localization of PLP is regulated by Rho GTPases and tyrosine kinases

As a cellular model, we used the oligodendroglial cell line, Oli-neu, stably expressing PLP-EGFP (Trajkovic et al., 2006). The advantage of this system is that cell differentiation can be triggered synchronously by adding conditioned medium from primary neuronal cultures to the cells. This treatment leads to the formation of a branching network of cellular processes. The morphological differentiation of Oli-neu cells is accompanied by a reorganization of membrane trafficking as shown by the redistribution of PLP from $\mathrm{LE} / \mathrm{L}$ to the cell surface. This response was not observed when Oli-neu cells were cultured with conditioned medium from primary cultures of astrocytes (Fig. 1). One aim of our present work was to identify the molecular mechanisms of this regulation. To this end, we screened the effect of a wide range of small molecules on the distribution of PLP-EGFP in Oli-neu cells. The tyrosine kinase inhibitor, Genistein, and the Rho-kinase (ROCK) inhibitor, Y27632, led to a redistribution of PLP from Lamp-1-positive LE/Ls to the surface of the plasma membrane (Fig. 1). ROCK kinases are regulated by Rho; we therefore transfected Oli-neu cells with the C3 transferase to block the activity of Rho. Inhibition of Rho led to a similar redistribution of PLP (Fig. 1). These results suggest that the activity of Rho may be involved in the regulation of membrane trafficking in oligodendrocytes.

\section{Neurons regulate RhoA GTPase activity} in oligodendroglial cells

To investigate a possible influence of neurons on RhoA activity in Oli-neu cells, we determined the activity of RhoA in Olineu cells, which were treated with conditioned medium from neuronal cultures or untreated. RhoA activity was measured by affinity precipitation of active (GTP-bound) Rho from cell lysates using a GST fusion protein containing the Rho-binding domain of Rhotekin (Ren et al., 1999). We found that the stimulation of Oli-neu cells with neuronal medium decreased the activity of RhoA (Fig. $2 \mathrm{~A}$ ). To obtain further support for a downregulation of RhoA activity after cell differentiation, we took advantage of a recently developed FRET-based biosensor for RhoA activity (Raichu-RhoA1237X) (Yoshizaki et al., 2003). Raichu-RhoA monitors the balance between guanine nucleotide exchange factors and GTPase activating proteins. The GTP-loading of Raichu-RhoA leads to the intramolecular binding of GTP-RhoA to the effector protein, Rho effector protein kinase $\mathrm{N}$ (PKN), and brings CFP in closer proximity to yellow fluorescent protein (YFP), thereby leading to an increase in FRET. FRET efficiency was measured by donor

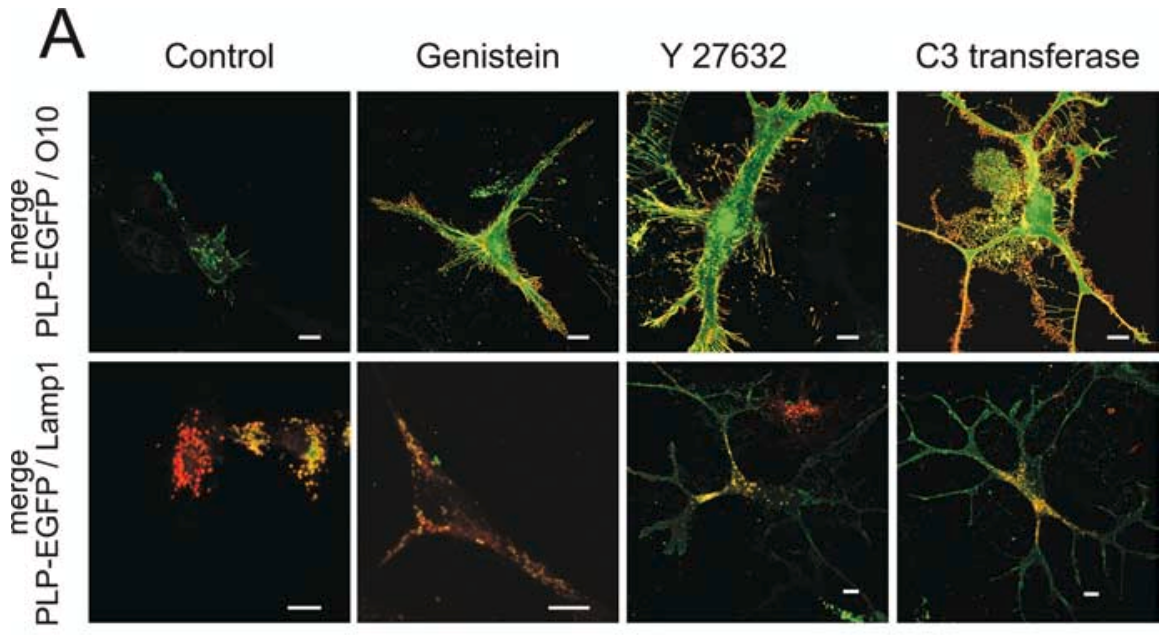

Figure 1. Subcellular localization of PLP is regulated by Rho GTPases and tyrosine kinases. Oli-neu cells stably expressing PLP-EGFP were treated for $16 \mathrm{~h}$ with Genistein, Y27632, C3 transferase, or conditioned medium from primary cultures of astroPLP on the cell surface (red) or by staining for Lamp-1 (red). Quantitative analysis of colocalization of PLP-EGFP with $010(\boldsymbol{B})$ and Lamp-1 (C) is shown. Values are given as the mean $\pm \mathrm{SE}\left(n>40\right.$ cells; ${ }^{*} p<0.05 ; t$ test). Scale bars, $10 \mu \mathrm{m}$.

dequenching after acceptor photobleaching. We found that coculturing Oli-neu cells with neurons or treatment of Oli-neu cells with conditioned neuronal medium reduced FRET efficiency compared with controls (Fig. $2 B$ ). These results with two independent approaches indicate that neurons reduce RhoA activity in oligodendroglial cells.

\section{Regulation of endocytosis by Rho GTPase and tyrosine} kinase activity

Internalization of plasma membrane can occur by different endocytic pathways, and several of those depend on the activity of Rho GTPases. We have shown previously that neurons reduce the endocytosis of PLP in oligodendrocytes (Trajkovic et al., 2006). We now determine whether the inhibition of endocytosis was specific for this particular pathway and, if so, how this pathway was regulated. To gain more insight in the regulation of endocy- 
A

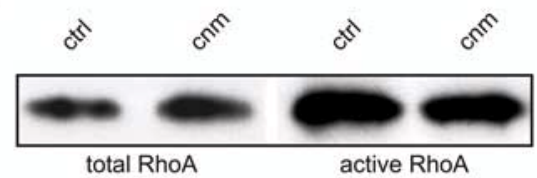

B

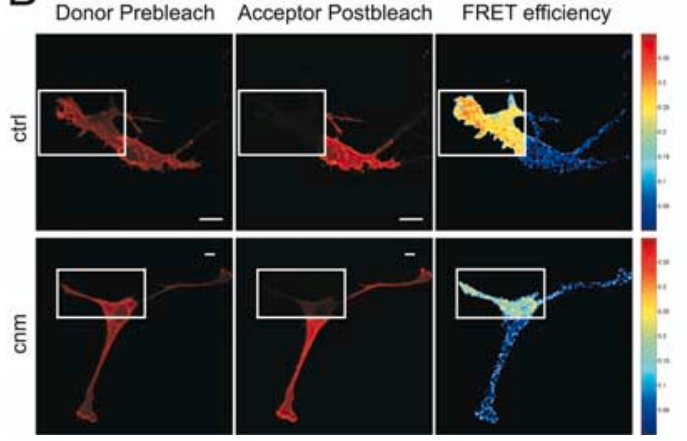

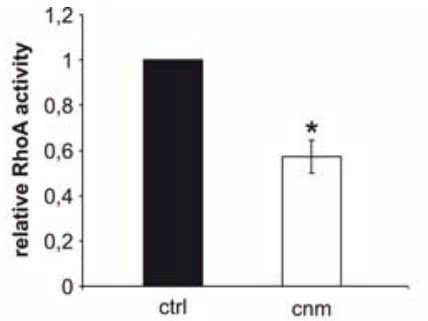

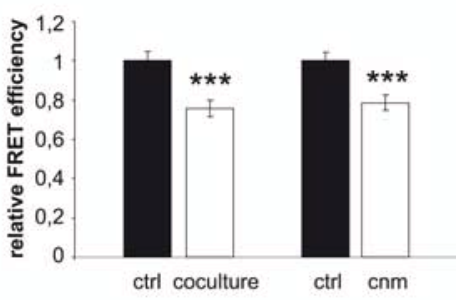

Figure 2. Neurons regulate RhoA GTPase activity in Oli-neu cells. $A, 0$ li-neu cells were grown for $\sim 8 \mathrm{~h}$ in the presence (cnm) or absence (ctrl) of conditioned medium from neuronal cultures. RhoA activity was measured by affinity precipitation of active (GTP-bound) Rho from cell lysates using a GST fusion protein containing the Rho-binding domain of Rhotekin. Precipitated proteins (active) and cell lysates (total) were immunoblotted with monoclonal antibody recognizing RhoA. Values are given as the mean \pm SE of an average of three independent experiments ( ${ }^{*} p<0.05 ; t$ test). $\boldsymbol{B}, 0$ li-neu cells were transfected with a plasmid encoding for Raichu-RhoA-1237X and grown for $\sim 8 \mathrm{~h}$ in the presence ( $\mathrm{cnm}$ ) or absence (ctrl) of conditioned medium from neuronal cultures or were added directly to primary neuronal cultures (coculture). Confocal fluorescence images of transfected Oli-neu cells are shown before (prebleach) and after (postbleach) photobleaching of the donor and acceptor, respectively. The acceptor was bleached in the region indicated by the rectangle. FRET efficiency was measured by donor dequenching after acceptor photobleaching and is represented in an efficiency map in pseudocolor (low to high FRET efficiency from blue to red). The error bars represent the means \pm SE $\left(n>25\right.$ cells; $^{* * *} p<0.001 ; t$ test). Scale bars, $10 \mu \mathrm{m}$

tosis in oligodendroglial cells, we started our analysis by examining receptor-mediated, clathrin-dependent, and fluid-phase endocytosis. Receptor-mediated, clathrin-dependent endocytosis was assessed by incubating Oli-neu cells for $30 \mathrm{~min}$ with Tf. Similar amounts of Tf were internalized in Oli-neu cells cultured with or without conditioned neuronal medium (Fig. 3). Subsequently, fluid-phase endocytosis was studied by feeding cells for $30 \mathrm{~min}$ with Dextran. Stimulation of Oli-neu cells by neuronal medium greatly inhibited the internalization of Dextran (Fig. 3). To examine by which pathway fluid-phase was taken up by oligodendroglial cells, we treated cells with various pharmacological inhibitors or expressed dominant-negative proteins to specifically interfere with the different endocytosis pathways. To inhibit clathrin-dependent internalization, we expressed a dominantnegative isoform of eps15 (EDelta/295) or dynaminII (dyn2K44A). Uptake of Dextran was not changed, although internalization of $\mathrm{Tf}$ was dramatically reduced (Fig. 3). These results indicated that fluid-phase did not depend on clathrin or dynaminII for endocytosis. Because most clathrin-independent pathways are sensitive to cholesterol or sphingolipid depletion, we used Fumonisin B1 (FB1) to selectively deplete sphingolipids (Cheng et al., 2006). Treatment of Oli-neu cells with FB1 did not affect the endocytosis of Tf but reduced the uptake of Dextran (Fig. 3). Both Rho GTPases and tyrosine kinases are known to play a regulatory role in clathrin-independent internalization pathways (Lamaze et al., 2001; Qualmann and Mellor, 2003; Damm et al., 2005). When Oli-neu cells were treated with the C3 transferase or Genistein, the uptake of Dextran was markedly reduced without affecting Tf internalization (Fig. 3).

To further explore the regulation of endocytosis, we studied the uptake of two lipid-raft markers, FITC-cholera toxin B sub- unit $(\mathrm{ChxB})$ and GPI-CFP. Both GPIanchored proteins and $\mathrm{ChxB}$ use clathrinindependent endocytosis pathways in most cells (Orlandi and Fishman, 1998; Sabharanjak et al., 2002; Kirkham et al. 2005). To analyze endocytosis of $\mathrm{ChxB}$ ChxB conjugated to FITC was bound to the cell surface at $4^{\circ} \mathrm{C}$, followed by incubation of the cells at $37^{\circ} \mathrm{C}$ for 40 min to allow transport to the Golgi complex. Using mask overlay image analysis, we quantified the internalization of FITC-ChxB to the GM130-positive Golgi apparatus. Compared with control cells, treatment with conditioned neuronal medium resulted in a significant inhibition in the delivery of FITC-ChxB to the Golgi (Fig. S1, available at www.jneurosci.org as supplemental material). To obtain further support for the inhibition of endocytosis by neuronal stimulation, we quantified the amount of FITC-ChxB being internalized during a $10 \mathrm{~min}$ uptake assay. Pretreatment of cells with conditioned neuronal medium reduced the uptake of $\mathrm{ChxB}$ significantly (Fig. S1, available at www.jneurosci.org as supplemental material). These findings support the conclusion that neurons regulate an endocytosis pathway used at least in part by ChxB. Next, we studied the endocytosis of GPI-CFP by performing antibody uptake experiments. In these experiments, internalized antibodies need to be distinguished from antibodies remaining at the cell surface. Because acidstripping and phosphatidylinositol-specific phospholipase C treatment were unable to fully remove anti-GFP antibody from the cell surface, we developed an alternative approach. Cells were labeled at $4^{\circ} \mathrm{C}$ with polyclonal antibodies against GFP (anti-GFP) and then warmed to $37^{\circ} \mathrm{C}$ to allow internalization of antibodies bound to GPI-CFP. Fixed, nonpermeabilized cells were labeled with Cy5-conjugated secondary antibodies to visualize GPI-CFP at the cell surface and then permeabilized and labeled with Cy3conjugated secondary antibodies to resolve internalized protein. Pretreatment of Oli-neu cells with neuronal medium led to a marked inhibition in endocytosis of GPI-CFP (Fig. $4 A$ ). In addition, and as observed for the uptake of Dextran, the treatment with Genistein and C3 transferase efficiently blocked endocytosis of GPI-CFP (Fig. 4A). Together, these data show that differentiation of Oli-neu cells is accompanied by a downregulation of an endocytosis pathway, which is used by GPI-linked fluorescent proteins and is responsible for the uptake of a major fraction of the fluid-phase. This pathway does not depend on clathrin function but requires tyrosine kinases and Rho activity.

\section{Endocytosed GPI-CFP is transported to LE/L in Oli-neu cells} In most cells, GPI-anchored proteins are routed from early endosomes into recycling endosomes before returning to the plasma membrane (Sabharanjak et al., 2002). Surprisingly, we found that a fraction of GPI-EGFP was present in LAMP-1positive LE/Ls in Oli-neu cells (Fig. $4 B$ ) as described previously for baby hamster kidney cells (Fivaz et al., 2002). Little colocalization of GPI-EYFP and Lamp-1 was seen in Oli-neu cells treated with conditioned neuronal medium (Fig. $4 B$ ). We followed the 


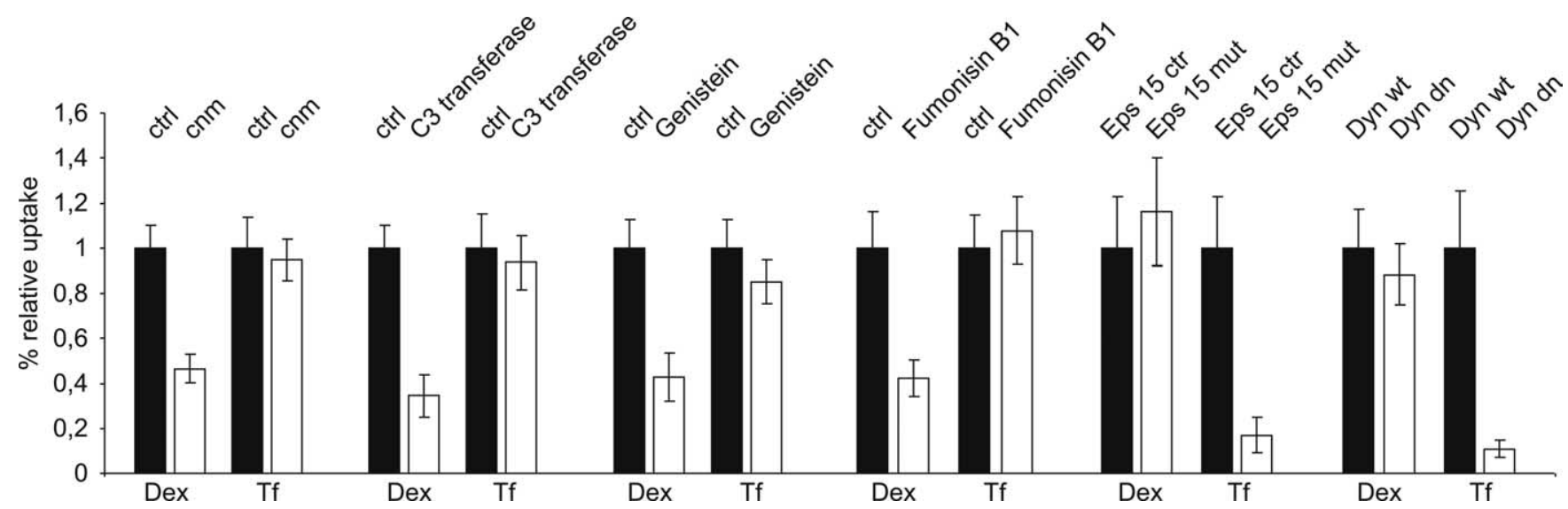

Figure 3. Regulation of clathrin-independent endocytosis by Rho GTPase and tyrosine kinase activity. Oli-neu cells were pretreated with Genistein (for $1 \mathrm{~h}$ ), $(3$ transferase (for $2 \mathrm{~h}$ ), conditioned neuronal medium (for 4 h), Fumonisin B1 (for 3 d), or transfected with plasmids encoding for Eps15III $\Delta 2$ (Eps15 ctrl), Eps15 E $\Delta 95 / 295$ (Eps15 mut), wild-type dynaminll (Dyn wt), or dominantnegative (K44A, Dyn dn). Receptor-mediated, clathrin-dependent endocytosis was assessed by incubating Oli-neu cells with Tf, and fluid-phase endocytosis was studied by feeding cells with Dextran-Rhodamine $10 \mathrm{~K}$ (Dex). Internalization $\left(30 \mathrm{~min}\right.$ at $37^{\circ} \mathrm{C}$ ) was quantified by image analysis. Values represent the means $\pm \mathrm{SE}(n>50$ cells).

endocytosis of GPI-CFP by antibody-internalization experiments and found that GPI-CFP was internalized from the plasma membrane to LE/Ls in Oli-neu cells (Fig. 4C).

\section{Regulation of LE/L dynamics by RhoB and c-Src activity}

We suggested previously that LE/Ls may have a specialized function in oligodendrocytes, namely to store and release membrane in a regulated manner (Trajkovic et al., 2006). Having established that Rho and tyrosine kinases regulate endocytosis in Oli-neu cells, we next asked whether they also regulate LE/L dynamics. In the absence of neurons, the majority of LE/Ls in oligodendrocytes are immobile and clustered perinuclear. When conditioned neuronal medium is added to Oli-neu cells, two pools of LE/Ls are observed: a perinuclear, immobile pool and a peripheral pool of highly mobile vesicles. When Oli-neu cells were treated with either C3 transferase or Genistein, we observed a redistribution of Lamp-1-positive LE/Ls to the cell periphery (Fig. 5A). To explore possible differences in the motility of LE/Ls, we performed fluorescence live imaging experiments by collecting images with a time interval of $15 \mathrm{~s}$ for PLP-EGFP and LysoTracker red. As shown previously, LE/Ls are primarily immobile in Oli-neu cells. Both treatments with Genistein or C3 transferase significantly increased the fraction of LE/Ls that moved during the time of the recordings (Fig. 5A). We investigated whether ROCK was involved in the regulation of LE/L motility by incubating cells with Y27632. We observed a significant increase in motility of LysoTracker-labeled vesicles (Fig. $5 B$ ). Similar results were obtained when primary cultures of oligodendrocytes were treated with Y27632 (Fig. 5C). These experiments suggest a role for Rho, ROCK, and tyrosine kinases in the regulation of LE/L dynamics in oligodendrocytes.

Higher vertebrates have three highly homologous Rho GTPases (RhoA, RhoB, and RhoC), which have different subcellular localizations and functions (Ridley, 2006). To test whether any of these proteins localizes to LE/Ls, we expressed HA-tagged wildtype, constitutively active and inactive versions of RhoA, RhoB, and RhoC, respectively. We found colocalization of both the wild-type and the constitutively active GTPase-deficient mutant (G14V) of RhoB with Lamp-1 (Fig. 5D). Predominantly cytoplasmic staining and some plasma membrane labeling, but no colocalization, with Lamp-1 was observed for RhoA and RhoC (data not shown). These results are consistent with previous stud- ies demonstrating a localization of RhoB to multivesicular endosomes (Robertson et al., 1995; Wherlock et al., 2004). We used a dominant-negative version of $\operatorname{RhoB}(\mathrm{T} 19 \mathrm{~N})$ to analyze whether RhoB is involved in the regulation of LE/L motility in Oli-neu cells. Transfection of dominant-negative RhoB led to a redistribution of a fraction of Lamp-1-positive LE/Ls to the cell periphery and increased the motility of the vesicles (Fig. $5 E$ ). To further confirm the role of RhoB in the regulation of endosome dynamics and to test for the specificity of the effects, we expressed the constitutively active forms of RhoA, RhoB, and RhoC in Oli-neu cells, in which the motility of endosomes had been induced by the incubation with conditioned neuronal medium. We found that all three Rho GTPases reduced the movement of LysoTrackerlabeled vesicles, but RhoB did this to the greatest extent (Fig. 5F). Because these results suggest a role for RhoB in the regulation of endosome dynamics, we measured the activity of RhoB in a Rhotekin pull-down assay after incubation of Oli-neu cells with conditioned neuronal medium. Similar to the results obtained with RhoA, we found that the levels of activated/GTP-bound RhoB decreased by conditioned neuronal medium compared with untreated cells (Fig. S2, available at www.jneurosci.org as supplemental material).

Recently, RhoB has been shown to regulate early endosome dynamics (Fernandez-Borja et al., 2005) and has been identified as a regulator of c-Src activity (Sandilands et al., 2004). We therefore tested the role of the tyrosine kinase, $\mathrm{c}-\mathrm{Src}$, in the regulation of LE/L dynamics. We began our analysis by determining the protein levels of Src in oligodendrocytes. We found that Oli-neu cells expressed Src and that levels of expression were reduced after treating cells for $16 \mathrm{~h}$ with conditioned neuronal medium (Fig. $6 A$ ). Next, we determined the c-Src kinase activity by phosphorylation of a specific c-Src peptide substrate. Conditioned neuronal medium reduced c-Src kinase activity compared with control cells, further suggesting a downregulation of Src function after treatment with conditioned neuronal medium (Fig. 6B)

We also detected Src in primary cultures of oligodendrocytes, and consistent with previous results (Colognato et al., 2004), the expression of Src was markedly reduced after morphological differentiation of oligodendrocytes (Fig. 6C).

We continued our analysis by determining the localization of c-Src in Oli-neu cells. Exogenously expressed active c-Src showed extensive colocalization with both PLP and Lamp-1, demonstrat- 

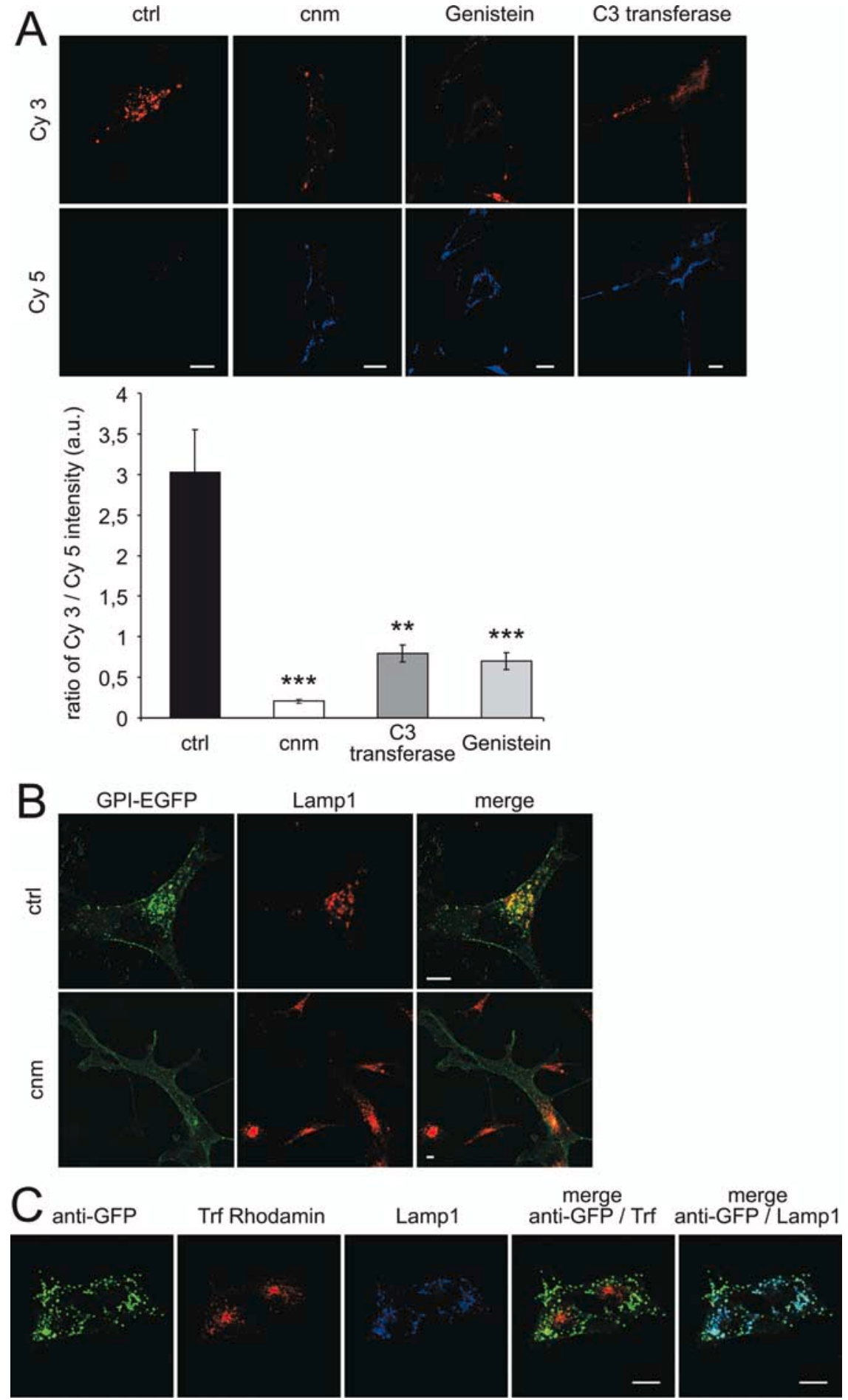

Figure 4. Neurons reduce the endocytosis of GPI-CFP to LE/Ls in Oli-neu cells. $\boldsymbol{A}$, Cells were treated with C3 transferase, Genistein, or conditioned neuronal medium ( $\mathrm{cnm}$ ) as indicated above (Fig. 3), and the uptake of GPI-CFP was analyzed by antibody uptake experiments. Cells were labeled at $4^{\circ} \mathrm{C}$ with a polyclonal antibody against GFP and then warmed to $37^{\circ} \mathrm{C}$ for 30 min to allow internalization of antibodies bound to GPI-CFP. Fixed, nonpermeabilized cells were labeled with Cy5-conjugated secondary antibody to visualize GPI-CFP at the cell surface (blue) and then permeabilized and labeled with Cy3-conjugated secondary antibodies to resolve internalized protein (red). By image analysis, we determined the ratio of Cy3- to Cy5-labeled GPI-CFP to obtain a value for the amount of protein internalized. Values represent the mean $\pm \mathrm{SE}\left(n>30\right.$ cells; ${ }^{* *} p<0.01 ;{ }^{* *} p<0.001 ; t$ test). $\boldsymbol{B}$; Oli-neu cells were transfected to express GPI-EGFP, incubated with conditioned neuronal medium (cnm) for 16 h or not incubated (ctrl), and treated with cycloheximid for the last $4 \mathrm{~h}$ of the incubation period to inhibit protein synthesis and to chase GPI-EGFP from the biosynthetic pathway. Cells were processed and analyzed by immunofluorescence for Lamp-1 (red) and GPI-EGFP (green) colocalization. C, The endocytosis of GPI-CFP was followed by antibody-internalization experiments (for $45 \mathrm{~min}$ at $37^{\circ} \mathrm{C}$ ). Cells were labeled at $4^{\circ} \mathrm{C}$ with polyclonal antibodies against GFP (anti-GFP) and then warmed to $37^{\circ} \mathrm{C}$ to allow internalization of antibodies bound to GPI-CFP together with Tf. Cells were fixed, permeabilized, and labeled with Alexa 488-conjugated secondary antibodies to resolve internalized GPI-CFP. Note the colocalization of anti-GFP (green) with Lamp-1 (blue) but not with Tf. Scale bars, $10 \mu \mathrm{m}$. ing its LE/L localization in Oli-neu cells (Fig. 6D). We expressed active c-Src in primary cultures of oligodendrocytes and observed the same extent of colocalization with Lamp-1. In addition, we found that exogenously expressed active c-Src resulted in perinuclear clustering of LE/Ls in primary oligodendrocytes (Fig. $6 E$ ), suggesting a role for $\mathrm{Src}$ in the regulation of LE/L function.

To determine whether Src influences the motility of LE/L, we exogenously expressed the constitutively active form of c-Src in Oli-neu cells, which had been preincubated with conditioned neuronal medium. Expression of constitutively active c-Src resulted in perinuclear clustering of LE/Ls and in a reduction of the motility of LysoTracker-labeled vesicles (Fig. 6F). Thus, both changes in Rho and Src activity modulate the dynamics of LE/Ls in Olineu cells.

\section{Plasma membrane condensation in Oli-neu cells}

Our results indicate that differentiation of Oli-neu cells is not only accompanied by a downregulation of a clathrinindependent, sphingolipid/cholesteroldependent endocytosis pathway, but is also associated with the mobilization of late endosomal/lysosomal membrane possibly for transport back to the plasma membrane. Both mechanisms may result in a gradual accumulation of cholesterol and sphingolipids in the plasma membrane of Oli-neu cells.

To study whether differentiation of Oli-neu cells is accompanied by alterations in the lipid structure of the plasma membrane, we used the fluorescent probe Laurdan to visualize its lipid order. Laurdan is an environmentally sensitive dye that undergoes a shift in its peak emission wavelength from $\sim 500 \mathrm{~nm}$ in fluid membranes to $\sim 440 \mathrm{~nm}$ in ordered membranes (Bagatolli et al., 2003; Gaus et al., 2003). The fluorescence intensity of Laurdan was recorded simultaneously at both peak emission wavelengths, and the normalized ratio representing the GP was used to determine the lipid order in the cell membranes. Higher GP values indicate a more condensed and ordered membrane. We found that treatment of Olineu cells with conditioned neuronal medium resulted in a dramatic increase in the lipid order of the cell membrane (Fig. $7 A, B)$. Furthermore, the GP images revealed that the membrane in the cellular processes was more condensed and ordered than the membrane enclosing the cell body (Fig. 7C). When Oli-neu cells 


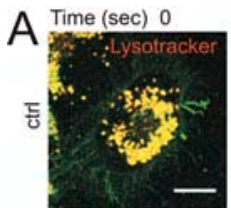

Time (sec) 0

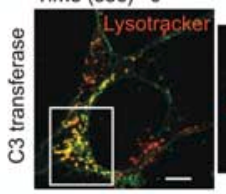

Time (sec) 0
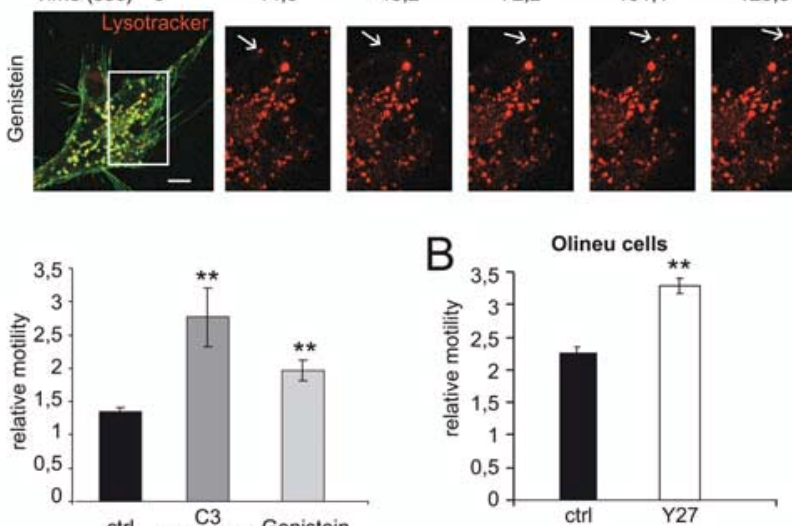

C primary oligodendrocytes

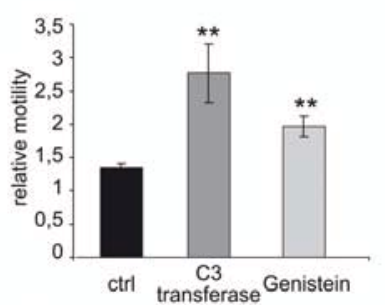

\section{PLP-EGFP}
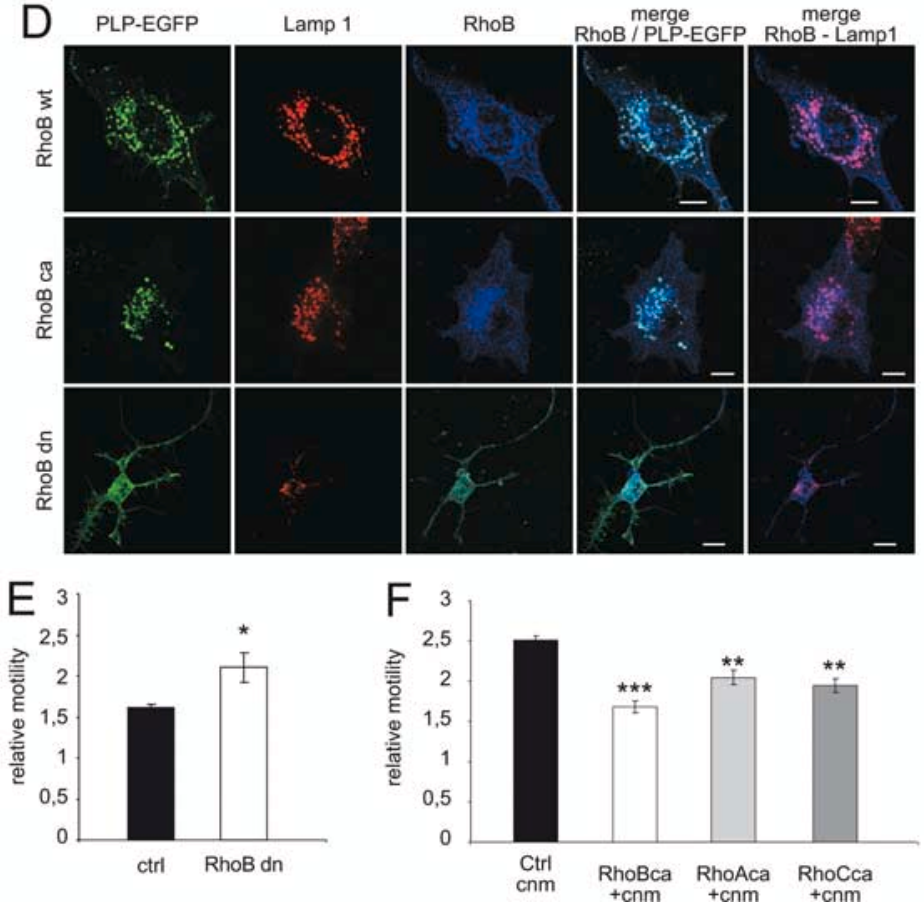

Figure 5. Regulation of LE/L dynamics by RhoB and tyrosine kinase activity. $\boldsymbol{A}$, Living Oli-neu cells expressing PLP-EGFP (green) were treated with C 3 transferase or Genistein, labeled with LysoTracker (red), and observed by confocal microscopy. Images were collected every $\sim 15 \mathrm{~s}$. A representative example of a time stack is shown. The images at the right of each time stack show a merged image (see Material and Methods) with the first image in red and movement over a $\sim 130$ s period in green. Relative motility of LE/Ls was quantified by determining the ratio of the integrated intensity of green to red pixels (see Material and Methods). Examples of moving vesicles are highlighted with arrows. Values represent the mean $\pm \mathrm{SE}\left(n>20\right.$ cells; ${ }^{* *} p<0.01$; $t$ test). $\boldsymbol{B}, \boldsymbol{C}$, Oli-neu cells $(\boldsymbol{B})$ and primary cultures of oligodendrocytes $(\boldsymbol{C})$ (for 2 d in culture) were pretreated with $10 \mu \mathrm{m} Y 27632$ for $2 \mathrm{~h}$ or left untreated, and the motility of LysoTracker-labeled vesicles was determined as in $\boldsymbol{A}$. Primary oligodendrocytes were identified by incubating living cells with 04 and Alexa 488-conjugated secondary antibody. Values represent the mean $\pm \mathrm{SE}(n>$ 20 cells; ${ }^{*} p<0.05 ;{ }^{* *} p<0.01 ; t$ test). D, Oli-neu cells were cotransfected to express PLP-EGFP and HA-tagged wild-type (wt), constitutively active (ca), and constitutively inactive ( $\mathrm{dn}$ ) versions of RhoB. Colocalization with PLP (green) and Lamp-1 (red) was observed for wild-type and the constitutively active versions of RhoB (blue). $\boldsymbol{E}$, Oli-neu cells were transfected to express membrane-targeted GFP (ctrl) or the dominant-negative version of RhoB (T19N) fused to GFP (RhoB dn), labeled with LysoTracker were treated with the C3 transferase, we also observed an increase in the GP values predominately in the membrane protrusions (Fig. $7 A, C$ ).

\section{Discussion}

We show that changes in Rho activity are responsible for switching between two modes of membrane transport in Oli-neu cells. In immature cells Rho is required for the function of a clathrin-independent endocytosis pathway that is used to transport membrane to LE/L. After morphological differentiation of Oli-neu cells, Rho activity is reduced, which is accompanied by the specific downregulation of the clathrin-independent endocytosis pathway and the mobilization of $\mathrm{LE} / \mathrm{L}$ vesicles.

What is the physiological function of this regulation? The formation of myelin requires the synthesis of a huge amount of membrane with a specific lipid composition. We therefore propose that the downregulation of this sphingolipid/ cholesterol-dependent endocytosis pathway represents a mechanism to control the plasma membrane composition of oligodendrocytes. This mechanism may contribute to the generation of myelin with its exceptionally high content of galactosylceramide and cholesterol. The reduction of this specific form of endocytosis may lead to a gradual accumulation of myelin membrane components within the plasma membrane of oligodendrocytes. Indeed, by using Laurdan to visualize the lipid packing, we observed a significant increase in the lipid order of the plasma membrane after treatment of the cells with conditioned neuronal medium or $\mathrm{C} 3$ transferase. We have shown recently that the formation of myelin in neuron-glia cocultures is associated with a condensation of the plasma membrane and that myelinbasic protein (MBP), a protein essential for myelin biogenesis, was involved in this process of membrane rearrangement (Fitzner et al., 2006). It is interesting that Oli-neu cells, which do not form compact myelin and only express small amounts of MBP, start to condense their membrane

\section{$\leftarrow$}

red, and imaged by confocal microscopy. Changes in mobility were determined as described in $\boldsymbol{A}$. Values represent the mean \pm SE ( $n>20$ cells; ${ }^{*} p<0.05 ; t$ test). $\boldsymbol{F}$, 0li-neu cells were transfected to express membrane-targeted GFP (ctrl) or the constitutively active versions of RhoB, RhoA, and RhoC fused to GFP treated with conditioned neuronal medium (cnm) for $12 \mathrm{~h}$ and labeled with LysoTracker red, and changes in mobility were determined as described in $A$. Values represent the mean \pm SE $\left(n>20\right.$ cells; ${ }^{* *} p<0.01 ;{ }^{* *} p<$ $0.001 ; t$ test). Scale bars, $10 \mu \mathrm{m}$. 
A

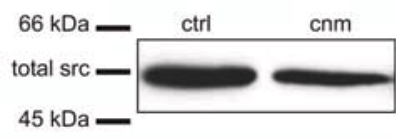

$\mathrm{C}_{+ \text {blocking pep }}^{\text {early pOL }}$

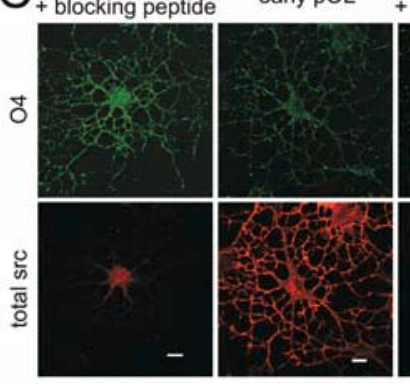

early pOL

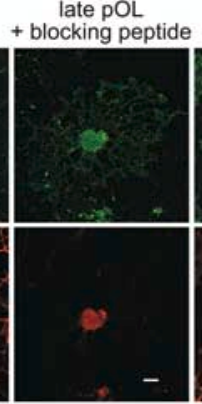

tide
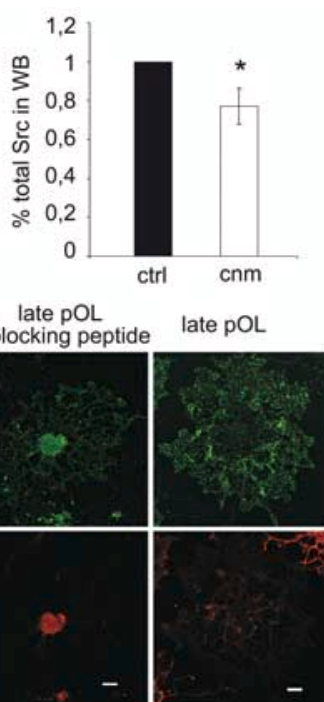

B
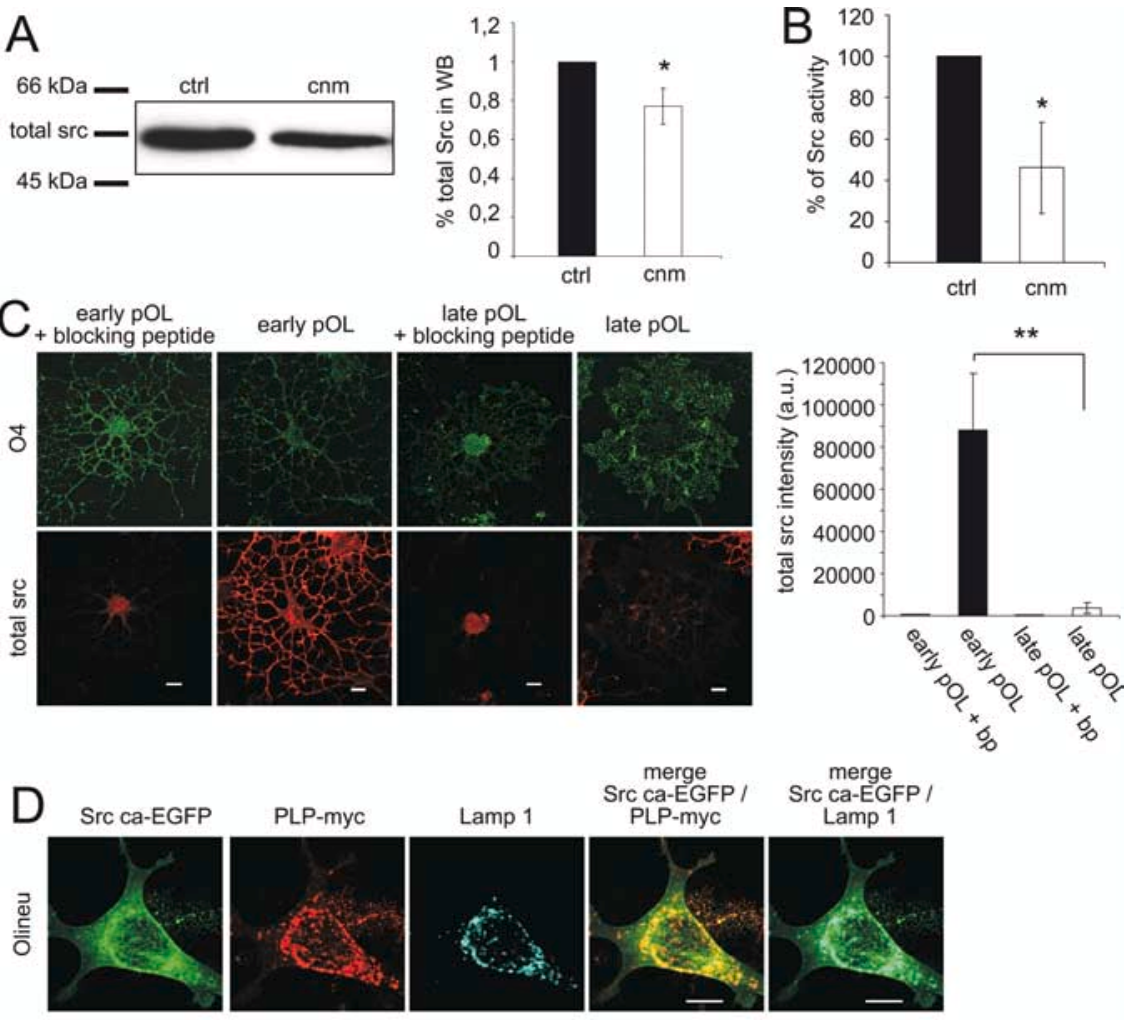

Lamp 1

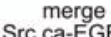

Src ca-EGFP/

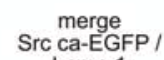

PLP-myc

Lamp 1
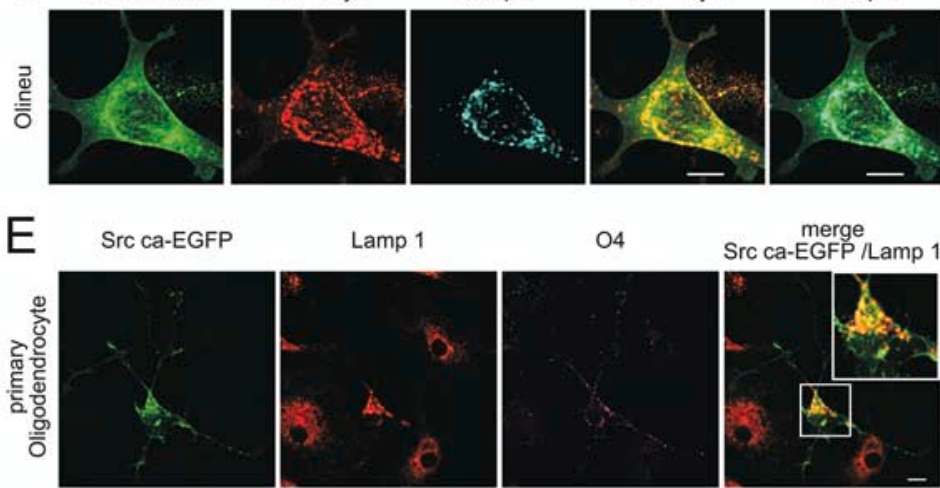

Lamp 1

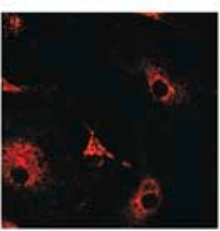

O4

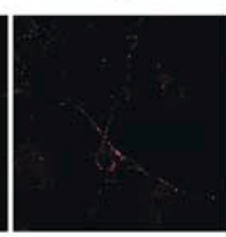
Src ca-EGFP /Lamp

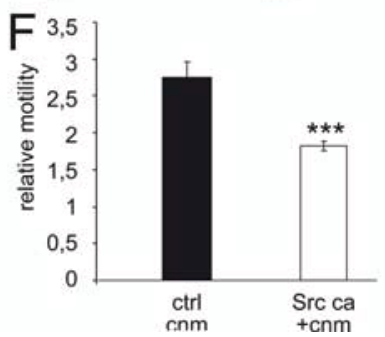

Figure 6. Changes in c-Src activity regulate the dynamics of LE/L. $A$, Oli-neu cells were incubated with conditioned neuronal medium (cnm) for $16 \mathrm{~h}$ or left untreated, cell lysates were prepared, and equal amounts of total protein were loaded and separated by SDS-PAGE. Src levels were determined by Western blot analysis. Values are presented as the mean \pm SD ( $n=4$ experiments; ${ }^{*} p<0.05$; $t$ test). $\boldsymbol{B}$, To determine the activity of $c-S r c$, Oli-neu cells were treated with conditioned neuronal medium or were untreated, and the cell lysates were immunoprecipitated with anti-Src antibodies and subjected to kinase assays. Results are expressed as the mean \pm SD of four experiments ( ${ }^{*} p<0.05 ; t$ test). C, Primary oligodendrocytes (p0L) were cultured for $2-5 \mathrm{~d}$, and levels of Src were determined at an early stage (cells with processes) and at a later stage of differentiation (cells with sheets) by quantifying immunofluorescence intensities. The specificity of the signal was demonstrated by preincubation with a blocking peptide (bp). Values are presented as the mean $\pm \mathrm{SE}\left(n>15\right.$ cells; ${ }^{* *} p<0.01 ; t$ test). $\boldsymbol{D}, 0$ li-neu cells were cotransfected with plasmids encoding for PLP-myc and constitutively active c-Src-EGFP (Src ca). E, Primary oligodendrocytes were transfected to express constitutively active c-Src-EGFP (Src ca). Immunofluorescence analysis reveals colocalization of c-Src-EGFP with Lamp-1. Note also the perinuclear clustering of LE/Ls. F, Oli-neu cells were transfected with membrane-targeted GFP (ctrl) or the constitutively active c-Src (Src ca), treated with conditioned neuronal medium, labeled with LysoTracker red, and imaged by confocal microscopy. Relative motility of LE/Ls was determined as described in Figure $3 A$. Values represent the mean $\pm S E(n>20$ cells; *** $p<0.001 ; t$ test). Scale bars, $10 \mu \mathrm{m}$.

after incubation with conditioned neuronal medium or Rho inactivation. The changes in membrane transport described in this study may contribute to the formation of condensed membrane domains in the plasma membrane of Oli-neu cells. Another, but not mutually exclusive, possibility is an increase in the biosynthesis of specific lipids and/or proteins after Rho inactivation or after receiving conditioned neuronal medium.

During development, oligodendrocytes regulate the composition as well as the size of their plasma membrane. Cells have, in general, a high rate of endocytosis. In fact, most cells internalize their entire cell surface within a few hours and keep a large fraction of their membrane in intracellular endosomal pools (Bomsel et al., 1989). In Oli-neu cells, the inactivation of Rho or the treatment with conditioned neuronal medium did not only reduce fluid-phase uptake but also increased the cell surface area. It is tempting to speculate that the regulation of cell size involves the balance between exocytosis and endocytosis. After differentiation, oligodendrocytes seem to shift this balance toward a high rate of exocytosis and a low rate of endocytosis, possibly to extend their surface, which eventually becomes $\sim 10,000$ times greater than that of a typical animal cell (Pfeiffer et al., 1993).

Previous work has shown that extracellular matrix components, such as laminin-2, promote extensive membrane extension in oligodendrocytes through integrin interactions (Buttery and ffrenchConstant, 1999; Chun et al., 2003; Colognato et al., 2004). The specific downregulation of a clathrin-independent endocytosis pathway by attachment to the extracellular matrix is interesting in this aspect (del Pozo et al., 2005). PLP, which forms a signaling complex with integrins in oligodendrocytes, may directly participate in the regulation of its own endocytosis by binding to the extracellular matrix (Gudz et al., 2002, 2006). Interestingly, a recent study shows that changes in the extracellular matrix regulate intracellular vesicular trafficking pathways in oligodendrocytes (Siskova et al., 2006).

The clathrin-independent endocytosis pathway that we identified in Oli-neu cells may be related to a previously described clathrin- and caveolin-independent pinocytic pathway (Sabharanjak et al., 2002) or to a closely related pathway implicated in the uptake of interleukin 2 receptors (Lamaze et al., 2001) and sodium pumps (Schmalzing et al., 1995). Because these pathways work constitutively and have a preference for specific lipids, they may have a very important role in the regulation of plasma membrane size and composition. The family of Rho GTPases are particularly well positioned to participate in this process of surface area homeostasis. Rho GTPases are able to respond to specific cues from the extracellular environment and 

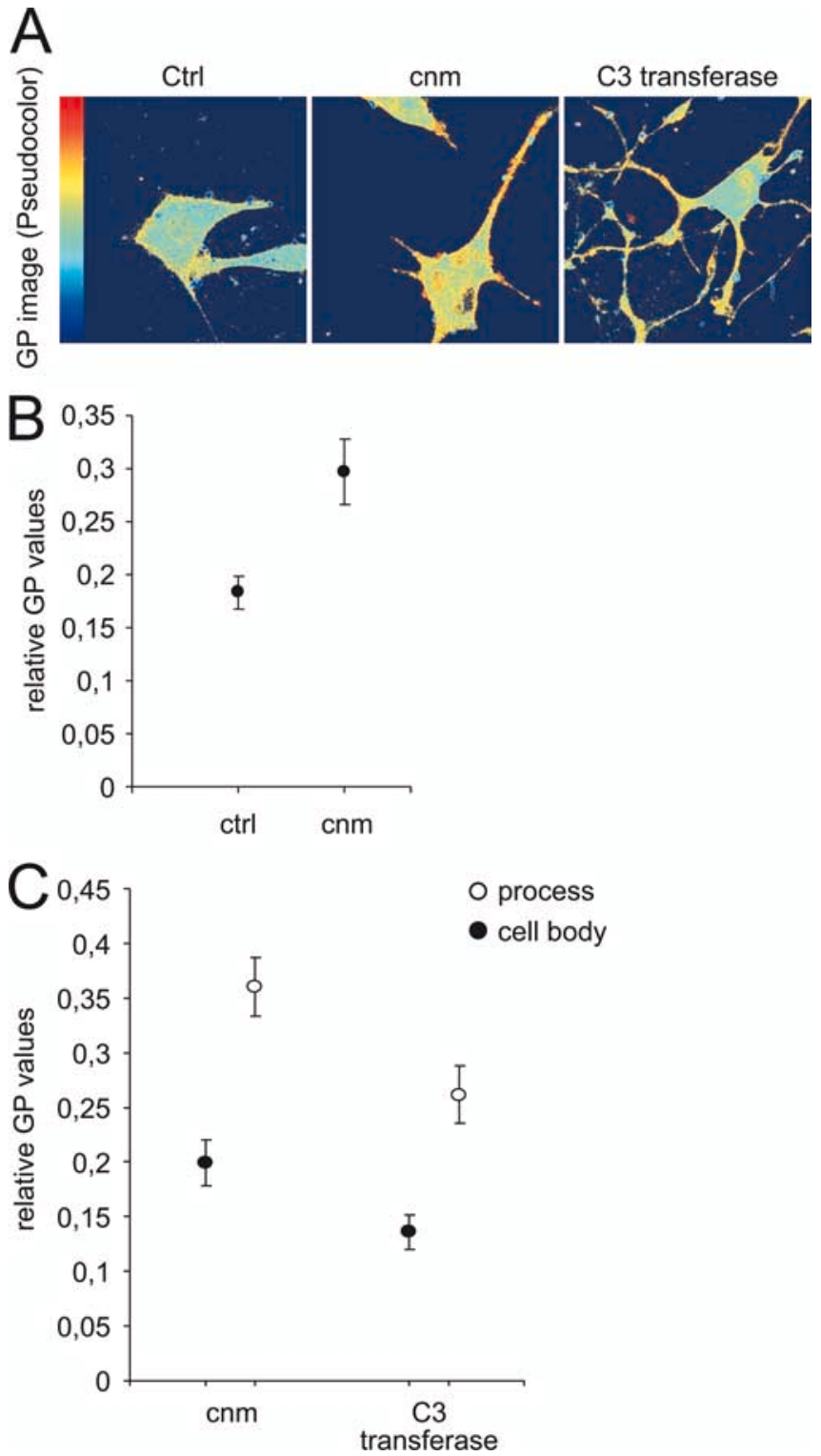

Figure 7. Neurons and RhoGTPase inactivation trigger plasma membrane condensation in Oli-neu cells. A, Oli-neu cells were left untreated (ctrl) or treated with (3 transferase or conditioned neuronal medium (cnm) for $16 \mathrm{~h}$, labeled with Laurdan, fixed, and processed for microscopy analysis. GP values were calculated from the Laurdan intensity images and pseudocolored as indicated (low to high GP values from blue to red). $\boldsymbol{B}$, GP values at the cell borders (3-6 pixels) were measured ( $n>100$ values from $>15$ cells), and values were presented in the graph as the means $\pm 2 \mathrm{SE}(p<0.001)$. C, For cells treated with conditioned neuronal medium and $(3$ transferase, GP values were measured for the membrane in the processes and for membrane enclosing the cell body. The GP values differed significantly $(p<0.001)$, indicating a polarization of the cell surface.

to coordinate the cellular response by linking membrane with actin dynamics (Ridley, 2006).

It is interesting that Rho and tyrosine kinases not only regulated the transport of cargo to LE/Ls but also controlled the dynamics of LE/Ls. LE/Ls may have a specialized function in oligodendrocytes, namely to store and release membrane after request. The observations that a large fraction of PLP accumulates undegraded in LE/Ls (Simons et al., 2002; Trajkovic et al., 2006) and that degradation of PLP involves the proteasome system (Kramer-Albers et al., 2006; McLaughlin et al., 2006) are consistent with this function. We found that RhoB-dependent and c-Src-dependent signaling pathways are responsible for the mo- bilization of LE/Ls. A RhoD-hDia2C-Src signaling cascade has been shown previously to regulate early endosome dynamics, suggesting that different RhoGTPases use similar effectors to control the motility of distinct populations of endosomes (Gasman et al., 2003). Additional work will be required to determine whether the mobilization of LE/Ls to the plasma membrane by Rho inactivation triggers LE/L membrane exocytosis in oligodendrocytes. Interestingly, regulated exocytosis of LE/Ls has been implicated recently in the regulation of process outgrowth and arborization in neurons (Arantes and Andrews, 2006).

Our results stress the important role of Rho in vesicular trafficking and demonstrate how changes in Rho activity can induce a switch between two modes of membrane transport. This work, together with previous studies, suggests that Rho inactivation is a critical factor to initiate the growth of myelin membrane in oligodendrocytes (Liang et al., 2004; Mi et al., 2005). Signaling from integrins to Fyn and Rho may play a critical role in the regulation of this pathway (Osterhout et al., 1999; Wolf et al., 2001; Liang et al., 2004). In contrast, the activity of other Rho GTPases, Cdc42 and Racl, seems to increase as differentiation proceeds, and recent evidence suggests that its activity is required to regulate a late stage in myelin formation (Liang et al., 2004; Thurnherr et al., 2006). Interestingly, in Schwann cells of the peripheral nervous system, the Rho-ROCK pathway is not inactivated during myelination as in oligodendrocytes (Melendez-Vasquez et al., 2001). Instead, it is switched on in Schwann cell microvilli, where it seems to play an important role in regulating the myelination of peripheral nerves (Gatto et al., 2003; Melendez-Vasquez et al., 2004). It will now be important to determine how the Rho GTPases are regulated and to identify the neuronal signals that reduce Rho activity in oligodendrocytes.

It is intriguing that neurons and oligodendrocytes seem to use Rho-kinase-dependent mechanisms for bidirectional communication. It is well known that many oligodendrocyte-derived inhibitors limit axonal regeneration by activating a RhoA signaling pathway in neurons (Yiu and $\mathrm{He}, 2006$ ). Likewise, it is feasible that Rho is turned on in oligodendroglial cells in diseases such as multiple sclerosis, in which the differentiation of oligodendrocytes is blocked in the vicinity of demyelinating lesions. Therefore, more knowledge in the regulation of Rho in oligodendrocytes may not only provide new insights into the development of myelin but also in the pathogenesis of myelin-related diseases.

\section{References}

Arantes RM, Andrews NW (2006) A role for synaptotagmin VII-regulated exocytosis of lysosomes in neurite outgrowth from primary sympathetic neurons. J Neurosci 26:4630-4637.

Bagatolli LA, Sanchez SA, Hazlett T, Gratton E (2003) Giant vesicles, Laurdan, and two-photon fluorescence microscopy: evidence of lipid lateral separation in bilayers. Methods Enzymol 360:481-500.

Barres BA, Raff MC (1999) Axonal control of oligodendrocyte development. J Cell Biol 147:1123-1128.

Bomsel M, Prydz K, Parton RG, Gruenberg J, Simons K (1989) Endocytosis in filter-grown Madin-Darby canine kidney cells. J Cell Biol 109:3243-3258.

Buttery PC, ffrench-Constant C (1999) Laminin-2/integrin interactions enhance myelin membrane formation by oligodendrocytes. Mol Cell Neurosci 14:199-212.

Cheng ZJ, Singh RD, Sharma DK, Holicky EL, Hanada K, Marks DL, Pagano RE (2006) Distinct mechanisms of clathrin-independent endocytosis have unique sphingolipid requirements. Mol Biol Cell 17:3197-3210.

Chun SJ, Rasband MN, Sidman RL, Habib AA, Vartanian T (2003) Integrinlinked kinase is required for laminin-2-induced oligodendrocyte cell spreading and CNS myelination. J Cell Biol 163:397-408.

Colognato H, Ramachandrappa S, Olsen IM, ffrench-Constant C (2004) In- 
tegrins direct Src family kinases to regulate distinct phases of oligodendrocyte development. J Cell Biol 167:365-375.

Damm EM, Pelkmans L, Kartenbeck J, Mezzacasa A, Kurzchalia T, Helenius A (2005) Clathrin- and caveolin-1-independent endocytosis: entry of simian virus 40 into cells devoid of caveolae. J Cell Biol 168:477-488.

Debruin LS, Harauz G (2007) White matter rafting-membrane microdomains in myelin. Neurochem Res 32:213-228.

del Pozo MA, Balasubramanian N, Alderson NB, Kiosses WB, Grande-Garcia A, Anderson RG, Schwartz MA (2005) Phospho-caveolin-1 mediates integrin-regulated membrane domain internalization. Nat Cell Biol 7:901-908.

Dubois-Dalcq M, Behar T, Hudson L, Lazzarini RA (1986) Emergence of three myelin proteins in oligodendrocytes cultured without neurons. J Cell Biol 102:384-392.

Dugas JC, Tai YC, Speed TP, Ngai J, Barres BA (2006) Functional genomic analysis of oligodendrocyte differentiation. J Neurosci 26:10967-10983.

Edgar JM, McLaughlin M, Yool D, Zhang SC, Fowler JH, Montague P, Barrie JA, McCulloch MC, Duncan ID, Garbern J, Nave KA, Griffiths IR (2004) Oligodendroglial modulation of fast axonal transport in a mouse model of hereditary spastic paraplegia. J Cell Biol 166:121-131.

Fernandez-Borja M, Janssen L, Verwoerd D, Hordijk P, Neefjes J (2005) RhoB regulates endosome transport by promoting actin assembly on endosomal membranes through Dia1. J Cell Sci 118:2661-2670.

Fitzner D, Schneider A, Kippert A, Mobius W, Willig KI, Hell SW, Bunt G, Gaus K, Simons M (2006) Myelin basic protein-dependent plasma membrane reorganization in the formation of myelin. EMBO J 25:5037-5048.

Fivaz M, Vilbois F, Thurnheer S, Pasquali C, Abrami L, Bickel PE, Parton RG, van der Goot FG (2002) Differential sorting and fate of endocytosed GPI-anchored proteins. EMBO J 21:3989-4000.

Gasman S, Kalaidzidis Y, Zerial M (2003) RhoD regulates endosome dynamics through Diaphanous-related Formin and Src tyrosine kinase. Nat Cell Biol 5:195-204.

Gatto CL, Walker BJ, Lambert S (2003) Local ERM activation and dynamic growth cones at Schwann cell tips implicated in efficient formation of nodes of Ranvier. J Cell Biol 162:489-498.

Gaus K, Gratton E, Kable EP, Jones AS, Gelissen I, Kritharides L, Jessup W (2003) Visualizing lipid structure and raft domains in living cells with two-photon microscopy. Proc Natl Acad Sci USA 100:15554-15559.

Gaus K, Le Lay S, Balasubramanian N, Schwartz MA (2006) Integrinmediated adhesion regulates membrane order. J Cell Biol 174:725-734.

Gielen E, Baron W, Vandeven M, Steels P, Hoekstra D, Ameloot M (2006) Rafts in oligodendrocytes: evidence and structure-function relationship. Glia 54:499-512.

Gudz TI, Schneider TE, Haas TA, Macklin WB (2002) Myelin proteolipid protein forms a complex with integrins and may participate in integrin receptor signaling in oligodendrocytes. J Neurosci 22:7398-7407.

Gudz TI, Komuro H, Macklin WB (2006) Glutamate stimulates oligodendrocyte progenitor migration mediated via an $\alpha \mathrm{v}$ integrin/myelin proteolipid protein complex. J Neurosci 26:2458-2466.

Jung M, Kramer E, Grzenkowski M, Tang K, Blakemore W, Aguzzi A, Khazaie K, Chlichlia K, von Blankenfeld G, Kettenmann H, et al (1995) Lines of murine oligodendroglial precursor cells immortalized by an activated neu tyrosine kinase show distinct degrees of interaction with axons in vitro and in vivo. Eur J Neurosci 7:1245-1265.

Jung M, Sommer I, Schachner M, Nave KA (1996) Monoclonal antibody O10 defines a conformationally sensitive cell-surface epitope of proteolipid protein (PLP): evidence that PLP misfolding underlies dysmyelination in mutant mice. J Neurosci 16:7920-7929.

Kirkham M, Fujita A, Chadda R, Nixon SJ, Kurzchalia TV, Sharma DK, Pagano RE, Hancock JF, Mayor S, Parton RG (2005) Ultrastructural identification of uncoated caveolin-independent early endocytic vehicles. J Cell Biol 168:465-476.

Kramer-Albers EM, Gehrig-Burger K, Thiele C, Trotter J, Nave KA (2006) Perturbed interactions of mutant proteolipid protein/DM20 with cholesterol and lipid rafts in oligodendroglia: implications for dysmyelination in spastic paraplegia. J Neurosci 26:11743-11752.

Lamaze C, Dujeancourt A, Baba T, Lo CG, Benmerah A, Dautry-Varsat A (2001) Interleukin 2 receptors and detergent-resistant membrane domains define a clathrin-independent endocytic pathway. Mol Cell 7:661-671.

Liang X, Draghi NA, Resh MD (2004) Signaling from integrins to Fyn to
Rho family GTPases regulates morphologic differentiation of oligodendrocytes. J Neurosci 24:7140-7149.

McLaughlin M, Barrie JA, Karim S, Montague P, Edgar JM, Kirkham D, Thomson CE, Griffiths IR (2006) Processing of PLP in a model of Pelizaeus-Merzbacher disease/SPG2 due to the rumpshaker mutation. Glia 53:715-722.

Melendez-Vasquez CV, Rios JC, Zanazzi G, Lambert S, Bretscher A, Salzer JL (2001) Nodes of Ranvier form in association with ezrin-radixin-moesin (ERM)-positive Schwann cell processes. Proc Natl Acad Sci USA 98:1235-1240.

Melendez-Vasquez CV, Einheber S, Salzer JL (2004) Rho kinase regulates Schwann cell myelination and formation of associated axonal domains. J Neurosci 24:3953-3963.

Mi S, Miller RH, Lee X, Scott ML, Shulag-Morskaya S, Shao Z, Chang J, Thill G, Levesque M, Zhang M, Hession C, Sah D, Trapp B, He Z, Jung V, McCoy JM, Pepinsky RB (2005) LINGO-1 negatively regulates myelination by oligodendrocytes. Nat Neurosci 8:745-751.

Milner RJ, Lai C, Nave KA, Lenoir D, Ogata J, Sutcliffe JG (1985) Nucleotide sequences of two mRNAs for rat brain myelin proteolipid protein. Cell 42:931-939.

Mirsky R, Winter J, Abney ER, Pruss RM, Gavrilovic J, Raff MC (1980) Myelin-specific proteins and glycolipids in rat Schwann cells and oligodendrocytes in culture. J Cell Biol 84:483-494.

Orlandi PA, Fishman PH (1998) Filipin-dependent inhibition of cholera toxin: evidence for toxin internalization and activation through caveolaelike domains. J Cell Biol 141:905-915.

Osterhout DJ, Wolven A, Wolf RM, Resh MD, Chao MV (1999) Morphological differentiation of oligodendrocytes requires activation of Fyn tyrosine kinase. J Cell Biol 145:1209-1218.

Pfeiffer SE, Warrington AE, Bansal R (1993) The oligodendrocyte and its many cellular processes. Trends Cell Biol 3:191-197.

Qualmann B, Mellor H (2003) Regulation of endocytic traffic by Rho GTPases. Biochem J 371:233-241.

Ren XD, Kiosses WB, Schwartz MA (1999) Regulation of the small GTPbinding protein Rho by cell adhesion and the cytoskeleton. EMBO J 18:578-585.

Ridley AJ (2006) Rho GTPases and actin dynamics in membrane protrusions and vesicle trafficking. Trends Cell Biol 16:522-529.

Robertson D, Paterson HF, Adamson P, Hall A, Monaghan P (1995) Ultrastructural localization of ras-related proteins using epitope-tagged plasmids. J Histochem Cytochem 43:471-480.

Sabharanjak S, Sharma P, Parton RG, Mayor S (2002) GPI-anchored proteins are delivered to recycling endosomes via a distinct cdc42-regulated, clathrin-independent pinocytic pathway. Dev Cell 2:411-423.

Salzer JL (2003) Polarized domains of myelinated axons. Neuron 40:297-318.

Sandilands E, Cans C, Fincham VJ, Brunton VG, Mellor H, Prendergast GC, Norman JC, Superti-Furga G, Frame MC (2004) RhoB and actin polymerization coordinate Src activation with endosome-mediated delivery to the membrane. Dev Cell 7:855-869.

Schafer DP, Rasband MN (2006) Glial regulation of the axonal membrane at nodes of Ranvier. Curr Opin Neurobiol 16:508-514.

Schmalzing G, Richter HP, Hansen A, Schwarz W, Just I, Aktories K (1995) Involvement of the GTP binding protein Rho in constitutive endocytosis in Xenopus laevis oocytes. J Cell Biol 130:1319-1332.

Schneider A, Lander H, Schulz G, Wolburg H, Nave KA, Schulz JB, Simons M (2005) Palmitoylation is a sorting determinant for transport to the myelin membrane. J Cell Sci 118:2415-2423.

Sherman DL, Brophy PJ (2005) Mechanisms of axon ensheathment and myelin growth. Nat Rev Neurosci 6:683-690.

Simons M, Trajkovic K (2006) Neuron-glia communication in the control of oligodendrocyte function and myelin biogenesis. J Cell Sci 119:4381-4389.

Simons M, Kramer EM, Thiele C, Stoffel W, Trotter J (2000) Assembly of myelin by association of proteolipid protein with cholesterol- and galactosylceramide-rich membrane domains. J Cell Biol 151:143-154.

Simons M, Kramer EM, Macchi P, Rathke-Hartlieb S, Trotter J, Nave KA, Schulz JB (2002) Overexpression of the myelin proteolipid protein leads to accumulation of cholesterol and proteolipid protein in endosomes/ lysosomes: implications for Pelizaeus-Merzbacher disease. J Cell Biol 157:327-336.

Siskova Z, Baron W, de Vries H, Hoekstra D (2006) Fibronectin impedes 
"myelin" sheet-directed flow in oligodendrocytes: a role for a beta 1 integrin-mediated PKC signaling pathway in vesicular trafficking. Mol Cell Neurosci 33:150-159.

Taylor CM, Marta CB, Claycomb RJ, Han DK, Rasband MN, Coetzee T, Pfeiffer SE (2004) Proteomic mapping provides powerful insights into functional myelin biology. Proc Natl Acad Sci USA 101:4643-4648.

Thurnherr T, Benninger Y, Wu X, Chrostek A, Krause SM, Nave KA, Franklin RJ, Brakebusch C, Suter U, Relvas JB (2006) Cdc42 and Rac1 signaling are both required for and act synergistically in the correct formation of myelin sheaths in the CNS. J Neurosci 26:10110-10119.

Trajkovic K, Dhaunchak AS, Goncalves JT, Wenzel D, Schneider A, Bunt G, Nave KA, Simons M (2006) Neuron to glia signaling triggers myelin membrane exocytosis from endosomal storage sites. J Cell Biol 172:937-948.

Wherlock M, Gampel A, Futter C, Mellor H (2004) Farnesyltransferase in- hibitors disrupt EGF receptor traffic through modulation of the RhoB GTPase. J Cell Sci 117:3221-3231.

Wolf RM, Wilkes JJ, Chao MV, Resh MD (2001) Tyrosine phosphorylation of p190 RhoGAP by Fyn regulates oligodendrocyte differentiation. J Neurobiol 49:62-78.

Wubbolts R, Fernandez-Borja M, Oomen L, Verwoerd D, Janssen H, Calafat J, Tulp A, Dusseljee S, Neefjes J (1996) Direct vesicular transport of MHC class II molecules from lysosomal structures to the cell surface. J Cell Biol 135:611-622.

Yiu G, He Z (2006) Glial inhibition of CNS axon regeneration. Nat Rev Neurosci 7:617-627.

Yoshizaki H, Ohba Y, Kurokawa K, Itoh RE, Nakamura T, Mochizuki N, Nagashima K, Matsuda M (2003) Activity of Rho-family GTPases during cell division as visualized with FRET-based probes. J Cell Biol 162: 223-232. 
3.3. Manuscript in preparation:

A novel pathway in the formation of intra-endosomal membrane and exosome biogenesis 
(work in progress)

\section{A novel pathway in the formation of intra-endosomal membrane and exosome biogenesis}

Katarina Trajkovic ${ }^{1,2^{\star}}$, Chieh $\mathrm{Hsu}^{1,2^{\star}}$, Dirk Wenzel ${ }^{3}$, Britta Brugger ${ }^{4}$ and Mikael Simons $s^{1,2}$

${ }^{1}$ Centre for Biochemistry and Molecular Cell Biology, University of Göttingen Humboldtallee 23, Göttingen, Germany

${ }^{2}$ Max-Planck-Institute for Experimental Medicine, Hermann-Rein-Str. 3, Göttingen, Germany

${ }^{3}$ Max-Planck-Institute for Biophysical Chemistry, Am Fassberg, Göttingen, Germany ${ }^{4}$ Heidelberg University Biochemistry Center (BZH), Im Neuenheimer Feld 328, Heidelberg, Germany

*equal contribution to first authorship

To whom correspondence should be addressed:

Dr. M. Simons, Centre for Biochemistry and Molecular Cell Biology, University of Göttingen

Humboldallee 23, 37073 Göttingen, Germany; phone: ++49-551-3899533; fax: ++49551-3899201; email: msimons@gwdg.de 
Sorting of ubiquitinated signalling receptors such as the EGF receptor (EGFR) into intraluminal vesicles (ILV) of multivesicular endosomes (MVE) depends on the endosomal sorting complex required for transport (ESCRT) machinery and is required for cargo degradation in lysosomes. Alternatively, ILV are secreted as exosomes into the extracellular milieu after fusion of MVEs with the plasma membrane. The mechanisms underlying the sorting of membrane into the different populations of ILVs are unknown. Here, we use an oligodendroglial cell line as a model system to address this question and show that the exosome-associated proteolipid protein (PLP) segregates together with lipidraft components from the EGFR into distinct microdomains on the endosomal membrane. We found that transfer of these microdomains into the lumen of the endosome does not depend on the function of the ESCRT-machinery. Furthermore, functional inhibition of components of the ESCRT-complex did not affect the release of PLP with exosomes. These results establish a novel pathway in ILV formation and exosome biogenesis.

After endocytosis, proteins and lipids destined for lysosomal degradation are first incorporated into intraluminal vesicles (ILV) of multivesicular endosomes (MVE) and are then delivered to lysosomes where the digestion of the intra-endosomal membrane takes place (Gruenberg and Stenmark, 2004; Piper and Katzmann, 2006). Alternatively, MVE can directly fuse with the plasma membrane leading to release of the ILV, or exosomes, to the extracellular environment, where they function in a multitude of intercellular signalling processes (Stoorvogel et al., 2002; Valadi et al., 2007; van Niel et al., 2006). How proteins and lipids are sorted to either the ILV that end up in lysosomes for degradation or to the ILV that escape degradation and are secreted as exosomes is an unresolved question.

To address this issue, we studied the membrane trafficking of the proteolipid protein (PLP) in Oli-neu cells, an oligodendroglial cell line that contains a large number of MVE (Trajkovic et al., 2006). PLP, the major protein of myelin of the central nervous system, is a palmitoylated, cholesterol-binding protein of $26 \mathrm{kD}$ that spans membrane four times and is mainly found in MVEs of Oli-neu cells (Simons et al., 2000; Trajkovic et al., 2006; Weimbs and Stoffel, 1992). To analyze whether PLP is released in association with exosomes, the cell culture medium of transiently 
transfected Oli-neu cells was subjected to sequential centrifugation steps with increasing centrifugal forces to obtain finally a $100000 \times \mathrm{g}$ pellet, which mainly contained small membrane vesicles with a size of approximately 50-100 nm (Fig. 1b), similar to previously described exosomes. Relatively large amounts of PLP were found in the $100000 \times \mathrm{g}$ pellet (Fig. 1a) and immunoelectron microscopy analysis revealed the presence of PLP on the vesicles (Fig. 1b). In contrast, PLP containing the cytotoxic, missense mutation $(\mathrm{A} 242 \mathrm{~V})$ that leads to misfolding of the protein and to its retention in the endoplasmic reticulum (ER) was not detected in the $100000 \times \mathrm{g}$ pellet (Fig. 1a), excluding cell lysis as a major contributing factor. To further rule out the possibility of membrane release as a consequence of cell lysis, the $100000 \times \mathrm{g}$ pellet was analyzed for the presence of calnexin, $\mathrm{Y}$-adaptin, GM130 and EEA1, membrane markers of the ER, TGN, cis-Golgi-matrix and early endosomes, respectively. These proteins were not detected in the $100000 \times \mathrm{g}$ pellet, which did contain the two exosomal marker proteins, Alix and flotillin (Fig. 1d). Furthermore, we exogenously expressed a variety of integral membrane proteins, the EGF receptor (EGFR), the vesicular stomatitis virus glycoprotein (VSV-G) (both a wild-type and a endocytosis-deficient mutant form) and the amyloid precursor proteins (APP), but did not detect any of these proteins in the $100000 x$ pellet, from which PLP was readily recovered, demonstrating the specificity of the sorting process (Fig. 1e). To further show the association of PLP with exosomes, we analyzed the $100000 \times \mathrm{g}$ pellet in a continuous sucrose density gradient and found that the majority of PLP was enriched in the same fraction as the exosomal marker protein Alix (Fig. 1c). Similar results were obtained when PLP-EGFP-stably expressing Oli-neu cells were used for the analysis (Fig. 1C). Exosomes are thought to be of endosomal origin and to derive by fusion of MVE with the cell surface. To explore whether PLP requires transport through the endosomal system for the release with exosomes, we cotransfected PLP with the early-endosomal GTPase-deficient Rab5 (Rab5 ${ }^{\text {Q79L }}$ ) to impair intraendosomal trafficking (Stenmark et al., 1994). We found that a large fraction of PLP was entrapped in the enlarged early endosomes and that the release of PLP by exosomes was significantly reduced (Fig. 1f).

These findings demonstrate that the membrane recovered from the $100000 \times \mathrm{g}$ pellets is highly enriched in exosomes that are of endosomal origin.

Previous studies have suggested the involvement of lipid rafts in the biogenesis of exosomes (de Gassart et al., 2003; Wubbolts et al., 2003). Therefore we determined 
the lipid composition of exosomes from Oli-neu cells by nano-electrospray ionization tandem mass spectrometry. The lipid composition was remarkably similar to the lipid composition that has been proposed for lipid rafts. We found that exosomes were not only enriched in cholesterol, but also contained higher amounts of sphingolipids (sphingomyelin, glucosylceramide and ceramide) and lower amounts of phophatidylcholine (PC) as compared to total cellular membrane (Fig. 2a). In addition, quantitative analysis of lipid subclasses revealed a striking increase in saturated $\mathrm{PC}$, at the expense of polyunsaturated species in the exosomal membrane fraction. Since incubation with polyunsaturated fatty acids (PUFAs) can disturb lipid raft function (Stulnig et al., 1998), we treated cells with PUFAs and analyzed the effect on exosome biogenesis. When Oli-neu cells were treated for three days with $50 \mu \mathrm{M}$ polyunsaturated eicosapentaenoic acid (20:5), a significant reduction of exosome release was observed for PLP as compared to control cells treated with stearic acid (18:0) (Fig. 2b). The treatment with eicosapentaenoic acid also did not result in the release of calnexin or $y$-adaptin into the exosomal membrane fraction.

Taken together our results suggest that raft lipids may play a role in exosome biogenesis. To further explore this possibility, we analyzed whether proteins that are targeted to exosomes use lipid microdomains to segregate from non-exosomal cargo in endosomes. To study endosomal microdomains, cells were transfected with Rab5 ${ }^{\mathrm{Q} 9 \mathrm{~L}}$ to enlarge early endosomes and to facilitate domain inspection by confocal immunofluorescence analysis (Raiborg et al., 2002). Previous studies have identified clathrin-coated microdomains on early endosomes that contain the ubiquitin-binding protein $\mathrm{Hrs}$ (hepatocyte-growth-factor regulated tyrosine kinase substrate), which sorts ubiquitinated proteins into these domains to mediate degradative protein sorting (Raiborg et al., 2006). The EGFR is a prototypic example of a receptor that requires Hrs-mediated sorting. To test whether PLP localizes to these microdomains, we analyzed the localization of PLP in Rab5 ${ }^{\mathrm{Q} 79 \mathrm{~L}}$ enlarged endosomes. There was little colocalization of PLP with either Hrs or EGFR (visualized by a 15 min incubation with Rhodamine-conjugated EGF), whereas Hrs colocalized to a large extent with the EGFR, consistent with previous studies (Fig. 3a,d). To characterize the nature of the PLP enriched domain, we analyzed the degree of colocalization with flotillin, a membrane scaffolding protein of non-caveolar lipid-raft microdomains. Confocal microscopy analysis revealed a strong colocalization of PLP with both endogenous and exogenously expressed flotillin (Fig. 3c). In contrast, there was little 
colocalization of flotillin with the EGFR (Fig. 3c). Likewise, we also found that GPIGL-GFP colocalized with PLP in the enlarged endosomes, again demonstrating lipidraft microdomain segregation (Fig. 3b).

The GTPase-deficient Rab5 ${ }^{\mathrm{Q} 79 \mathrm{~L}}$-enlarged endosomes are hybrid organelles that do not only contain early endosomal, but also late endosomal membrane, possibly because they recruit Rab7, but fail to displace Rab5 (Rink et al., 2005). We therefore wondered whether segregation of lipid-raft components may not only occur into different domains of these enlarged endosomes, but also into distinct endosomal populations. Indeed, we found that flotillin-containing late endosomes contained PLP (Fig. 4b) and were highly enriched in cholesterol as demonstrated by filipin staining (Fig. 4d) and also incorporated Bodipy-lactosylceramide after $60 \mathrm{~min}$ of internalization (Fig. 4e), but did not contain the late endosomal/lysosomal lipid lysobisphosphatidic acid (LBPA) that regulates intra-endosomal dynamics along the degradative pathway (Fig. 4f). Moreover, endocytosis assay revealed that GPI-GLGFP was targeted more efficiently to flotillin-containing endosomes as compared to the EGFR (Fig. 4c). Interestingly, endocytosed GPI-anchored proteins seem to be transported to late endosomes or recycling endosomes, depending on the lipid composition of the cell type (Fivaz et al., 2002). Collectively, our data support the idea that lipid rafts may contribute to the sorting into different domains on endosomes or even into different population of multivesicular endosomes.

Having shown that EGFR and PLP are found on distinct domains of Rab5 ${ }^{\mathrm{Q79L}}$ enlarged endosomes, we next addressed the question whether they use distinct pathways of inward budding into the lumen of endosomes. It is well established that sorting of EGFR into the ILV requires the sequential action of different components of the endosomal sorting complex required for transport (ESCRT) machinery (Hurley and Emr, 2006; Williams and Urbe, 2007). As an assay for inward budding, we determined the amount of EGF being transferred to the lumen of the enlarged endosomes after EGF stimulation. To interfere with the function of ESCRT machinery, we used either RNAi or dominant-negative mutants against Hrs, Tsg101, Alix or Vps4, respectively. An efficient knock-down of protein was obtained by performing two rounds of siRNA delivery using nucleofection (Fig. 5a). We found that depletion of either Hrs or Tsg101 significantly reduced the intraluminal transport of EGFR into enlarged endosomes, whereas Alix depletion only had a minor effect (Fig. $5 b)$. The reduction of EGF in the endosomal lumen correlated with a defect in EGF 
degradation in all experiments (Fig. S1). These results are consistent with previous data, validating our inward budding assay (Babst et al., 2000; Bache et al., 2003; Bishop and Woodman, 2000; Lloyd et al., 2002; Razi and Futter, 2006; Yoshimori et al., 2000).

To analyze the involvement of the ESCRT-machinery in the transfer of PLP into the lumen of enlarged endosomes, similar experiments were performed for PLP. We found that neither the knock-down of Hrs, Tsg101 nor Alix had any influence on the inward budding of PLP (Fig. 5b). In addition, the functional inhibition of the ESCRTmachinery did not change the colocalization of PLP with Lamp-1 (Fig. S2). Together, these results suggest the existence of an ESCRT-independent pathway for the transport of PLP into the lumen of endosomes. To obtain further support for this conclusion, we determined the amount of PLP being released in association with exosomes after interfering with the function of the ESCRT-machinery. Knock-down of Tsg101 and Alix, overexpression of Tsg101, or expression of dominant-negative ATP binding-defective form of Vps4 did not impair the secretion of PLP with exosomes (Fig. $5 c, d$ ). The assay was validated by showing that the secretion of GFP-tagged Gag protein of the Moloney murine leukemia virus with virus-like particles was strongly reduced by the expression of dominant-negative Vps4 (Fig. 5d) (Garrus et al., 2001) .

Collectively, our data show that PLP is transferred into the lumen of endosomes and released by exosomes in an ESCRT-independent fashion.

Sorting of membrane into the lumen of MVEs requires the lateral segregation of cargo at the limiting membrane, followed by the formation and incorporation of cargo into the inwardly budding vesicles. For ubiquitinated cargo it is well established that Hrs sorts these proteins into clathrin-coated microdomains through interaction with phosphatidylinositol (3)-phosphate on early endosomes, thereby recruiting downstream-ESCRT factors that initiate the inward budding of the domain into the lumen of MVEs. Here, we provide evidence for an alternative pathway, which is independent of the ESCRT machinery but seems to require lipid rafts as collecting devices for the lateral segregation of cargo in the limiting membrane of endosomes. Endocytosis, which includes Invagination of the plasma membrane, occurs by multiple mechanisms and creates different populations of endocytic carriers. The diversity of ILV populations in MVEs also points to different mechanisms of how proteins and lipids can enter endosomes. In the future it will be important to learn 
more about these different pathways and to find out whether they are active on the same endosome and/or on specialized subpopulations of endosomes in order to gain more insight in the function of intra-endosomal membrane trafficking . 


\section{Materials and Methods}

\section{Antibodies, plasmids, and other reagents}

The following plasmids were used: GFP-Rab5 and Rab5 ${ }^{\mathrm{Q79L}}$ (M. Zerial, MPI-CBG, Dresden), EGFR-EGFP (D. Arndt-Jovin, MPI for Bioph. Chem., Göttingen), GFPVps4(KQ) and GFP-hVps4(EQ) (P. Woodman, University of Manchester, UK), mycHrs ( $H$. Stenmark, Institute of Cancer Research, Norway), flotillin-1-GFP (Ben Nichols, MRC, Laboratory of Molecular Biology, Cambridge, UK), flotillin-2-GFP and flotillin-2-RFP (L. Rajendran, MPI-CBG, Dresden), CD63-EGFP (D. Cutler, University College London, UK). The pcDNA3-HA-Hrs vector was generated by insertion of the $\mathrm{HA}$ tag-containing oligonucleotides into the EcoRI and HindllI restriction sites of the pcDNA3-myc-Hrs plasmid. The following primary antibodies were used: anti-myc (monoclonal IgG; Cell Signalling; and polyclonal rabbit, Upstate), mouse monoclonal antibodies against flotillin-1 and flotillin-2, monoclonal rat anti-Lamp-1 (BD Biosciences, Heidelberg, Germany), mouse monoclonal against LBPA (J. Gruenberg, University of Geneva, Switzerland). Secondary antibodies were obtained from Dianova and GE Healthcare.

\section{Cell culture, transfections and siRNA delivery}

The oligodendroglial precursor cell line, Oli-neu (provided by J. Trotter, University of Mainz, Mainz, Germany), were cultured as described previously (Trajkovic et al., 2006). Transient transfections were performed using FuGENE transfection reagent (Roche) according to the manufacturer's protocol. siRNA was delivered into Oli-neu cells by nucleofection with Amaxa basic neuron kit according to the manufacturer's protocol and repeated after 48 hours, followed by another 48 hours of incubation before carrying out the experiment. The following siRNAs were used: sense r(GGAACUACUGGGAGAAGAA)dTdT and

antisense r(UUCUUCUCCCAGUAGUUCC)dGdG r(CUGUAUAAACAGAUUCUAA)dTdT

against Hrs

and

against and sense antisense r(UUAGAAUCUGUUUAUACAG)dTdG r(GAACCUGGAUAAUGAUGAA)dTdT sense antisense r(UUCAUCAUUAUCCAGGUUC)dTdG against Alix (Quiagen). Control siRNA was obtained from Ambion.

For modification of cellular lipids, cells were incubated for 2 days in serum-free medium supplemented with $0.4 \%(\mathrm{w} / \mathrm{v})$ bovine serum albumin (containing less than 
$0.003 \%$ total fatty acids) and $50 \mu \mathrm{M}$ of either polyunsaturated eicosapentaenoic acid (20:5 (n-3)) or stearic acid (18:0), as described (Stulnig et al., 1998); cells were then transiently transfected with PLP-myc and $4 \mathrm{~h}$ after transfection the fatty acid treatment was renewed and incubation was continued for $\sim 16$ hours.

\section{Microscopy and analysis}

Immunofluorescence was performed as described previously (Trajkovic et al., 2006). Methanol fixations were used in the case of labelling for flotillin. Fluorescence images were acquired on a microscope DMRXA, Leica or a confocal microscope LSM 510, Carl Zeiss Microlmaging, Inc. with a 63x oil plan-Apochromat objective (NA 1.4; Carl Zeiss Microlmaging, Inc.). Image processing and analysis were performed using Meta Imaging Series 6.1 software (Universal Imaging Corp.). Quantification of colocalization was performed with the colocalization module of the software. To assay EGF degradation, cells transfected with EGFR-EGFP were incubated with 50 $\mathrm{ng} / \mathrm{ml}$ Rhodamine-labelled EGF (Invitrogen) for $15 \mathrm{~min}$, washed, and incubated for 4 $\mathrm{h}$ in conditioned culture medium to allow EGF degradation. For quantification, images of randomly selected transfected cells were recorded at fixed settings below pixelvalue saturation and fluorescence intensities were quantified using Meta Imaging Series 6.1 software. For quantification of protein transfer into the lumen of Rab5 ${ }^{\mathrm{Q79L}}$ enlarged endosomes, confocal sections of enlarged endosomes were recorded using the GFP-Rab5 ${ }^{\mathrm{Q} 79 \mathrm{~L}}$ outline as a reference to obtain images through the middle region of the endosome. The fluorescence intensities within the lumen and the limiting membrane (indicated by the GFP-Rab5 ${ }^{\mathrm{Q} 9 \mathrm{~L}}$ outline) of the endosomes were measured and quantified using Meta Imaging Series 6.1 software.

For electron microscopy analysis, the exosomes were loaded onto a carbon-coated grids, fixed in $2 \%$ paraformaldehyde, washed and immunolabelled with anti-3F4 antibody followed by $10 \mathrm{~nm}$ gold-labelled secondary antibody (Sigma Aldrich). The exosomes were post-fixed in $2 \%$ glutaraldehyde, washed, contrasted in $2 \%$ uranyl acetate, embedded in a mixture of uranyl acetate and methyl cellulose and examined by an electron microscope.

\section{Exosome purification}

Exosomes from transiently or stably transfected Oli-neu cells were prepared as described (Fevrier et al., 2004). Briefly, before the exosome preparation, culture 
medium was replaced by serum-free medium. Cell culture media were collected and centrifuged twice for $5 \mathrm{~min}$ at $3000 \times g$ and $4500 \times g$, respectively, and ultracentrifuged at $10000 \times g$ for $30 \mathrm{~min}$ and at $100000 \times g$ for $1 \mathrm{~h}$. To prepare continuous sucrose gradients, the $100000 \times g$ pellet was resuspended in $2.5 \mathrm{M}$ sucrose in $20 \mathrm{mM}$ Hepes ( $\mathrm{pH} 7.4)$, and a step gradient of sucrose (2.25, 2.0, 1.75, $1.5,1.25,1.0,0.75,0.5$, and $0.25 \mathrm{M}$ ) was layered over the exosome-containing, 2.5 $\mathrm{M}$ sucrose solution. The gradient was spun at $200000 \times g$ for at least $16 \mathrm{~h}$ using a SW55 rotor. Fractions were collected from the top of the gradient, diluted with PBS, and spun at $100000 \times g$ with a TLA-100.3 rotor. Pellets were resuspended in sample buffer, subjected to $10 \%$ SDS-PAGE electrophoresis, and transferred to nitrocellulose membranes. Western blots were revealed by enhanced chemiluminescence (Amersham Pharmacia) and bands were quantified using the Image J Software.

\section{Lipid analysis}

Lipid analysis using Nano-ESI-MS/MS was performed as described previously (Brugger et al., 2006). 


\section{Figure legends}

\section{Figure 1}

Identification and characterization of exosomes. (a) Oli-neu cells were transiently transfected with myc-tagged, wild-type PLP (wt PLP) or mutant (A242V) (msd PLP) and switched to serum-free medium $\sim 16 \mathrm{~h}$ after transfection; the medium was collected after $\sim 6 \mathrm{~h}$ of further incubation before submitting it to sequential centrifugation steps as indicated. The resulting pellets of each centrifugation step were analyzed by Western blotting for PLP. (b) The $100000 \times$ g pellet was negatively stained with $1 \%$ uranyl acetate and immunolabeled with antibodies against PLP (right panel). Bar, $200 \mathrm{~nm}$. (c) A $0.25-2.5 \mathrm{M}$ sucrose gradient was loaded on top of the 100 $000 \times g$ pellet and the resulting fractions were analyzed for PLP from transiently (PLP-myc) or stably transfected (PLP-YFP) cells and for the exosomal marker protein, Alix. (d) Cell lysates and $100000 \times \mathrm{g}$ pellets (P100) were analyzed by Western blotting for the indicated proteins. (e) Cells were transiently transfected and cell lysates and $100000 \times \mathrm{g}$ pellets were analyzed for the respective proteins. (f) Cells were transiently co-transfected with either GFP-Rab5 ${ }^{\mathrm{Q79L}}$ or GFP-Rab5 and myc-PLP and the amount of PLP in the cell lysate and $100000 \times \mathrm{g}$ pellets was determined and quantified. Results are expressed as the mean \pm SD of five experiments $\left({ }^{* *} p<0.01 ; t\right.$ test). An immunofluorescence image of cells expressing GFP-Rab5 ${ }^{\mathrm{Q79L}}$ and myc-PLP is shown. Bar, $5 \mu \mathrm{m}$.

\section{Figure 2}

Lipid analysis. (a) Quantification of lipids from total cells (CM) and the $100000 \times \mathrm{g}$ pellets (P100) was performed by nano-electrospray ionization tandem mass spectrometry. Error bars represent standard deviation of the mean. (b) Oli-neu cells were treated with $50 \mu \mathrm{M}$ polyunsaturated eicosapentaenoic acid (20:5), stearic acid (18:0) or vehicle alone and the amount of PLP was determined in the cell lysate $(C L)$ and in the $100000 \times \mathrm{g}$ pellet (P100) of the sequential centrifugation steps of the culture medium. Results are expressed as the mean \pm SD of four experiments ${ }^{*} p<$ $0.05 ; t$ test).

\section{Figure 3}

Endosomal subdomain structure. (a-d) Oli-neu cells were co-transfected with Rab5 $^{\mathrm{Q} 79 \mathrm{~L}}$ and PLP-myc, EGFR, CFP-GPI, flotillin-2-RFP, HA-Hrs, CD63-RFP as 
indicated. Cells transfected with EGFR were incubated for 15 min with Rhodaminelabelled EGF. Cells were then processed and analyzed for immunofluorescence microscopy. A GFP antibody was used to stain CFP-GPI. Insets show the enlarged endosomes. Bar, $5 \mu \mathrm{m}$. The level of colocalization of the different proteins on endosome membranes was quantified as described in Materials and Methods.

\section{Figure 4}

Flotillin-containing endosomes. (a) Oli-neu cells were co-transfected with PLP-myc and EGFR, Rhodamine-labelled EGF was bound to the cell surface and internalized for $60 \mathrm{~min}$, before the cells were processed and analyzed by immunofluorescence microscopy. The boxed areas are show below in a higher magnification. (b) Cells were transfected with PLP-myc and stained with anti-myc and anti-flotillin-1 or -2 antibodies. (c) Cells were transfected with CFP-GPI or EGFR, anti-GFP or Rhodamine-labelled EGF was allowed to bind to the cell surface, respectively, and internalization was carried out for 30 to $120 \mathrm{~min}$. The level of colocalisation with flotillin-1 is shown in the graph. Values are expressed as the mean $\pm \mathrm{SD}(n>30$ cells; ${ }^{* * *} p<0.001 ; t$ test) (d) Cells were transfected with flotillin-2-GFP and stained with filipin to visualize free cholesterol. (e) BODIPY-lactosylceramide were added to transiently transfected flotillin-2-RFP cells as a BSA complex, cells were cultured for $45 \mathrm{~min}$ at $37^{\circ} \mathrm{C}$ in culture medium, washed and furthe $\mathrm{r}$ incubated for $1 \mathrm{~h}$ at $37^{\circ} \mathrm{C}$ in culture medium. Fluorescent lipid present at the plasma membrane was then removed by washing several times. (f) Cells were transfected with flotillin-1-GFP or flotillin-2-RFP and stained with anti-LBPA antibodies. Bar, $5 \mu \mathrm{m}$.

\section{Figure 5}

ESCRT-independent transport of PLP. (a) A control RNA duplex or siRNA against Hrs, Tsg101 or Alix was delivered by nucleofection into Oli-cells. The efficiency of the knock-down was demonstrated by Western blotting (upper panel). Actin or tubulin are shown as loading controls (lower panel). (b) RNAi treated cells were co-transfected with GFP-Rab5 ${ }^{\text {Q79L }}$ and EGFR (left panel) or PLP-myc (right panel). EGFR transfected cells were incubated for 15 min with Rhodamine-labelled EGF before fixation. Cells were then processed and analyzed for immunofluorescence microscopy. Quantification of the relative amount of PLP or EGF in the lumen as compared to the limiting membrane of the enlarged endosomes was quantified. 
Values represent the mean \pm SE $\left(n>70\right.$ endosomes; ${ }^{*} p<0.05 ;{ }^{* * *} p<0.001 ; t$ test $)$. (c) Cells are treated with siRNA and transfected with PLP-myc (left panel), or cotransfected with Tsg101 and PLP-myc (right panel). The amount of PLP was determined in the cell lysate $(C L)$ and in the $100000 \times$ g pellet (P100) of the sequential centrifugation steps of the culture medium. Results are expressed as the mean \pm SD of five experiments. (d) Cells were co-transfected with PLP-myc (upper panel) or Gag-GFP (lower panel) and different ratios of ATPase-defective Vps4(KQ). The amount of Gag-GFP and PLP-myc was determined in the cell lysate $(\mathrm{CL})$ and in the $100000 \times$ g pellet (P100). . Results are expressed as the mean \pm SD of three experiments $\left({ }^{* *} p<0.01 ;{ }^{* *} p<0.001 ; t\right.$ test $)$. Bar, $5 \mu \mathrm{m}$. 


\section{Supplementary Information}

\section{Figure S1}

EGF degradation after treatment with siRNA against Hrs, Tsg101 and Alix.

A control RNA duplex or siRNA against Hrs, Tsg101 or Alix was delivered by nucleofection into Oli-cells. Cells were transfected with EGFR, incubated with Rhodamine-labelled EGF for $15 \mathrm{~min}$, washed, and incubated further for $4 \mathrm{~h}$ in conditioned culture medium to allow EGF degradation. For quantification, images of randomly selected transfected cells were recorded and fluorescence intensities were quantified. Values represent the mean \pm SE $(n>70$ cells; * $p<0.05$; $t$ test) (b) Cells were co-transfected with EGFR and ATPase-defective Vps4(KQ) or wild-type GFP$\mathrm{hVps} 4(\mathrm{wt})$ and EGF degradation was determined. Values represent the mean $\pm \mathrm{SE}$ $(n>70$ cells).

\section{Figure S2}

Colocalization of PLP and Lamp-1 after treatment with siRNA against Hrs, Tsg101 and Alix.

A control RNA duplex or siRNA against Hrs, Tsg101 or Alix was delivered by nucleofection into Oli-cells. Cells were transfected with PLP-myc, processed and analyzed by immunofluorescence microscopy. Colocalization of PLP and Lamp-1 was determined. Values represent the mean \pm SE ( $n>30$ cells). Bar, $5 \mu \mathrm{m}$. 


\section{Acknowledgements}

We are grateful to J. Gruenberg, D. Caplan, M. Zerial, D. Arndt-Jovin, P. Keller and $\mathrm{H}$. Stenmark for providing reagents. R. White for helping establishing with RNAi experiments. We thank the directors of the MPI for Experimental Medicine for continued support. The work was supported by the Deutsche Forschungsgemeinschaft (SFB 523, GRK521). 


\section{References:}

Babst, M., Odorizzi, G., Estepa, E.J. and Emr, S.D. (2000) Mammalian tumor susceptibility gene 101 (TSG101) and the yeast homologue, Vps23p, both function in late endosomal trafficking. Traffic, 1, 248-258.

Bache, K.G., Brech, A., Mehlum, A. and Stenmark, H. (2003) Hrs regulates multivesicular body formation via ESCRT recruitment to endosomes. J Cell Biol, 162, 435-442.

Bishop, N. and Woodman, P. (2000) ATPase-defective mammalian VPS4 localizes to aberrant endosomes and impairs cholesterol trafficking. Mol Biol Cell, 11, 227-239.

Brugger, B., Glass, B., Haberkant, P., Leibrecht, I., Wieland, F.T. and Krausslich, H.G. (2006) The HIV lipidome: a raft with an unusual composition. Proc Natl Acad Sci U S A, 103, 2641-2646.

de Gassart, A., Geminard, C., Fevrier, B., Raposo, G. and Vidal, M. (2003) Lipid raft-associated protein sorting in exosomes. Blood, 102, 4336-4344.

Fevrier, B., Vilette, D., Archer, F., Loew, D., Faigle, W., Vidal, M., Laude, H. and Raposo, G. (2004) Cells release prions in association with exosomes. Proc Natl Acad Sci U S A, 101, 9683-9688.

Fivaz, M., Vilbois, F., Thurnheer, S., Pasquali, C., Abrami, L., Bickel, P.E., Parton, R.G. and van der Goot, F.G. (2002) Differential sorting and fate of endocytosed GPI-anchored proteins. Embo J, 21, 3989-4000.

Garrus, J.E., von Schwedler, U.K., Pornillos, O.W., Morham, S.G., Zavitz, K.H., Wang, H.E., Wettstein, D.A., Stray, K.M., Cote, M., Rich, R.L., Myszka, D.G. and Sundquist, W.I. (2001) Tsg101 and the vacuolar protein sorting pathway are essential for HIV-1 budding. Cell, 107, 55-65.

Gruenberg, J. and Stenmark, H. (2004) The biogenesis of multivesicular endosomes. Nat Rev Mol Cell Biol, 5, 317-323.

Hurley, J.H. and Emr, S.D. (2006) The ESCRT complexes: structure and mechanism of a membranetrafficking network. Annu Rev Biophys Biomol Struct, 35, 277-298.

Lloyd, T.E., Atkinson, R., Wu, M.N., Zhou, Y., Pennetta, G. and Bellen, H.J. (2002) Hrs regulates endosome membrane invagination and tyrosine kinase receptor signaling in Drosophila. Cell, 108, 261-269.

Piper, R.C. and Katzmann, D.J. (2006) Biogenesis and Function of Multivesicular Bodies. Annu Rev Cell Dev Biol.

Raiborg, C., Bache, K.G., Gillooly, D.J., Madshus, I.H., Stang, E. and Stenmark, H. (2002) Hrs sorts ubiquitinated proteins into clathrin-coated microdomains of early endosomes. Nat Cell Biol, $\mathbf{4}$, 394-398.

Raiborg, C., Wesche, J., Malerod, L. and Stenmark, H. (2006) Flat clathrin coats on endosomes mediate degradative protein sorting by scaffolding Hrs in dynamic microdomains. J Cell Sci, 119, 2414-2424.

Razi, M. and Futter, C.E. (2006) Distinct roles for Tsg101 and Hrs in multivesicular body formation and inward vesiculation. Mol Biol Cell, 17, 3469-3483.

Rink, J., Ghigo, E., Kalaidzidis, Y. and Zerial, M. (2005) Rab conversion as a mechanism of progression from early to late endosomes. Cell, 122, 735-749. 
Simons, M., Kramer, E.M., Thiele, C., Stoffel, W. and Trotter, J. (2000) Assembly of myelin by association of proteolipid protein with cholesterol- and galactosylceramide-rich membrane domains. J Cell Biol, 151, 143-154.

Stenmark, H., Parton, R.G., Steele-Mortimer, O., Lutcke, A., Gruenberg, J. and Zerial, M. (1994) Inhibition of rab5 GTPase activity stimulates membrane fusion in endocytosis. Embo J, 13, 1287-1296.

Stoorvogel, W., Kleijmeer, M.J., Geuze, H.J. and Raposo, G. (2002) The biogenesis and functions of exosomes. Traffic, 3, 321-330.

Stulnig, T.M., Berger, M., Sigmund, T., Raederstorff, D., Stockinger, H. and Waldhausl, W. (1998) Polyunsaturated fatty acids inhibit $\mathrm{T}$ cell signal transduction by modification of detergentinsoluble membrane domains. J Cell Biol, 143, 637-644.

Trajkovic, K., Dhaunchak, A.S., Goncalves, J.T., Wenzel, D., Schneider, A., Bunt, G., Nave, K.A. and Simons, M. (2006) Neuron to glia signaling triggers myelin membrane exocytosis from endosomal storage sites. J Cell Biol, 172, 937-948.

Valadi, H., Ekstrom, K., Bossios, A., Sjostrand, M., Lee, J.J. and Lotvall, J.O. (2007) Exosomemediated transfer of mRNAs and microRNAs is a novel mechanism of genetic exchange between cells. Nat Cell Biol.

van Niel, G., Porto-Carreiro, I., Simoes, S. and Raposo, G. (2006) Exosomes: a common pathway for a specialized function. $J$ Biochem (Tokyo), 140, 13-21.

Weimbs, T. and Stoffel, W. (1992) Proteolipid protein (PLP) of CNS myelin: positions of free, disulfidebonded, and fatty acid thioester-linked cysteine residues and implications for the membrane topology of PLP. Biochemistry, 31, 12289-12296.

Williams, R.L. and Urbe, S. (2007) The emerging shape of the ESCRT machinery. Nat Rev Mol Cell Biol, 8, 355-368.

Wubbolts, R., Leckie, R.S., Veenhuizen, P.T., Schwarzmann, G., Mobius, W., Hoernschemeyer, J., Slot, J.W., Geuze, H.J. and Stoorvogel, W. (2003) Proteomic and biochemical analyses of human $B$ cell-derived exosomes. Potential implications for their function and multivesicular body formation. J Biol Chem, 278, 10963-10972.

Yoshimori, T., Yamagata, F., Yamamoto, A., Mizushima, N., Kabeya, Y., Nara, A., Miwako, I., Ohashi, M., Ohsumi, M. and Ohsumi, Y. (2000) The mouse SKD1, a homologue of yeast Vps4p, is required for normal endosomal trafficking and morphology in mammalian cells. Mol Biol Cell, 11, 747-763. 
a

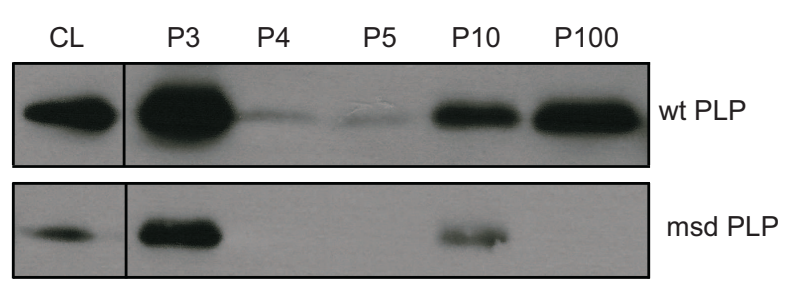

Sucrose concentration

C $0.25 \mathrm{M}$

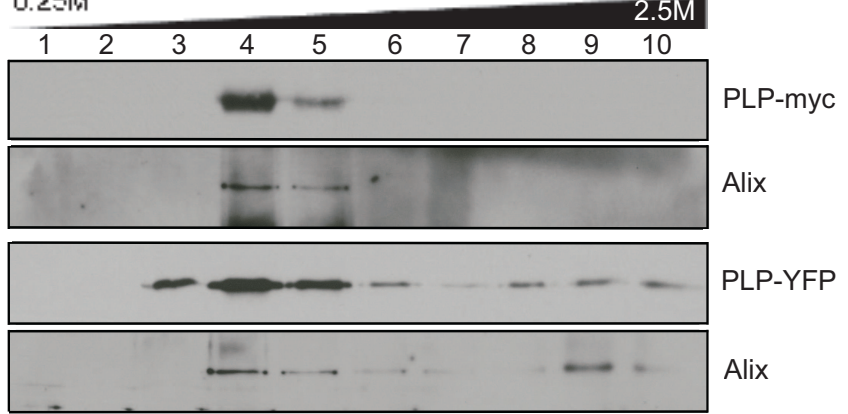

e
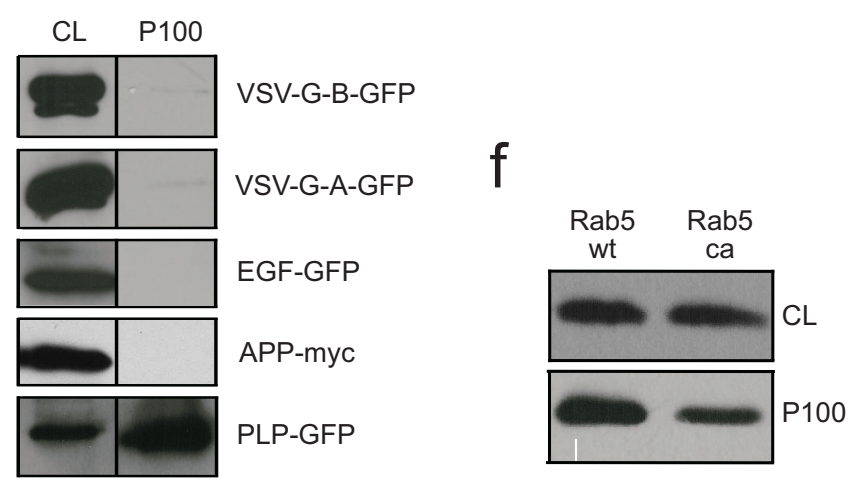

b negative stain
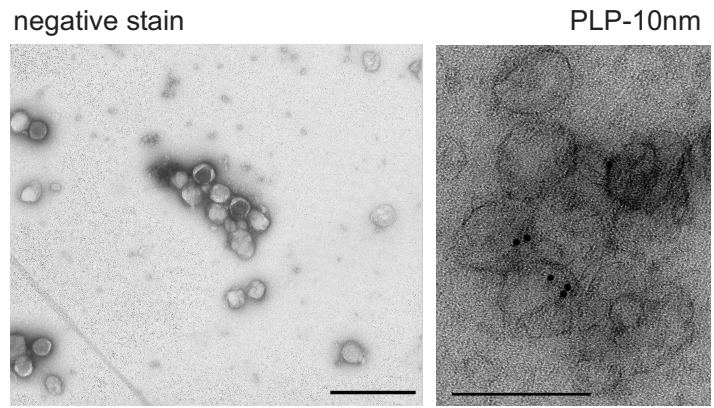

d
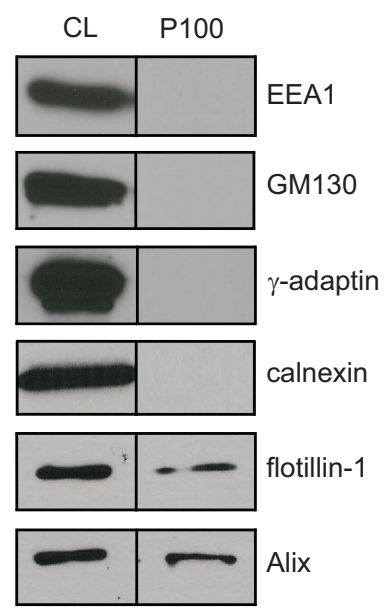
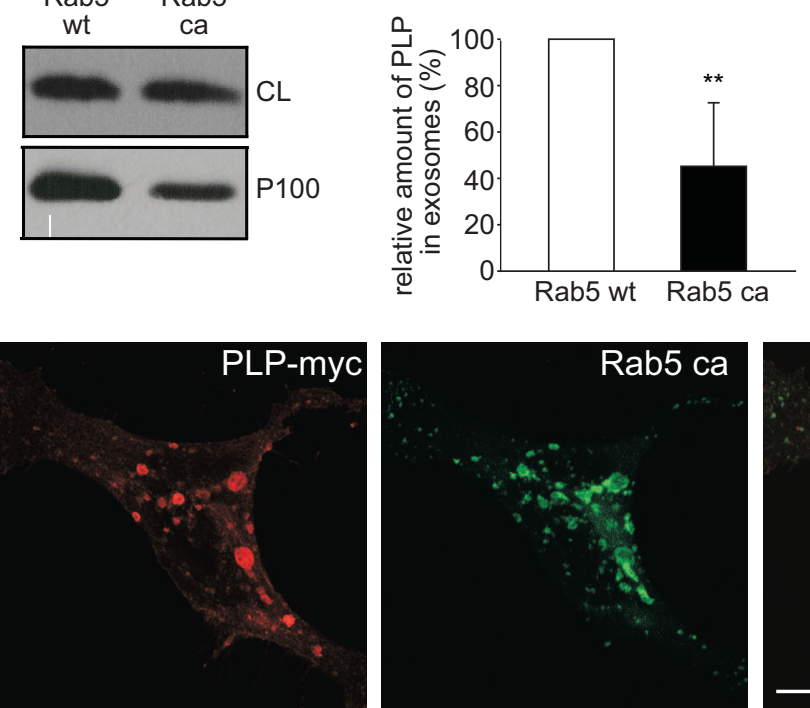

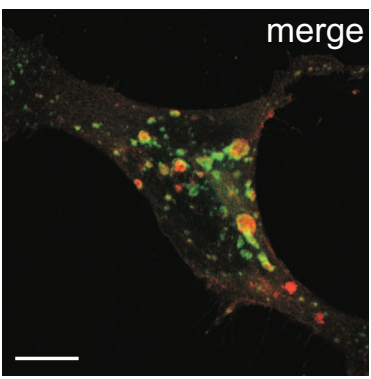

Figure 1 
a
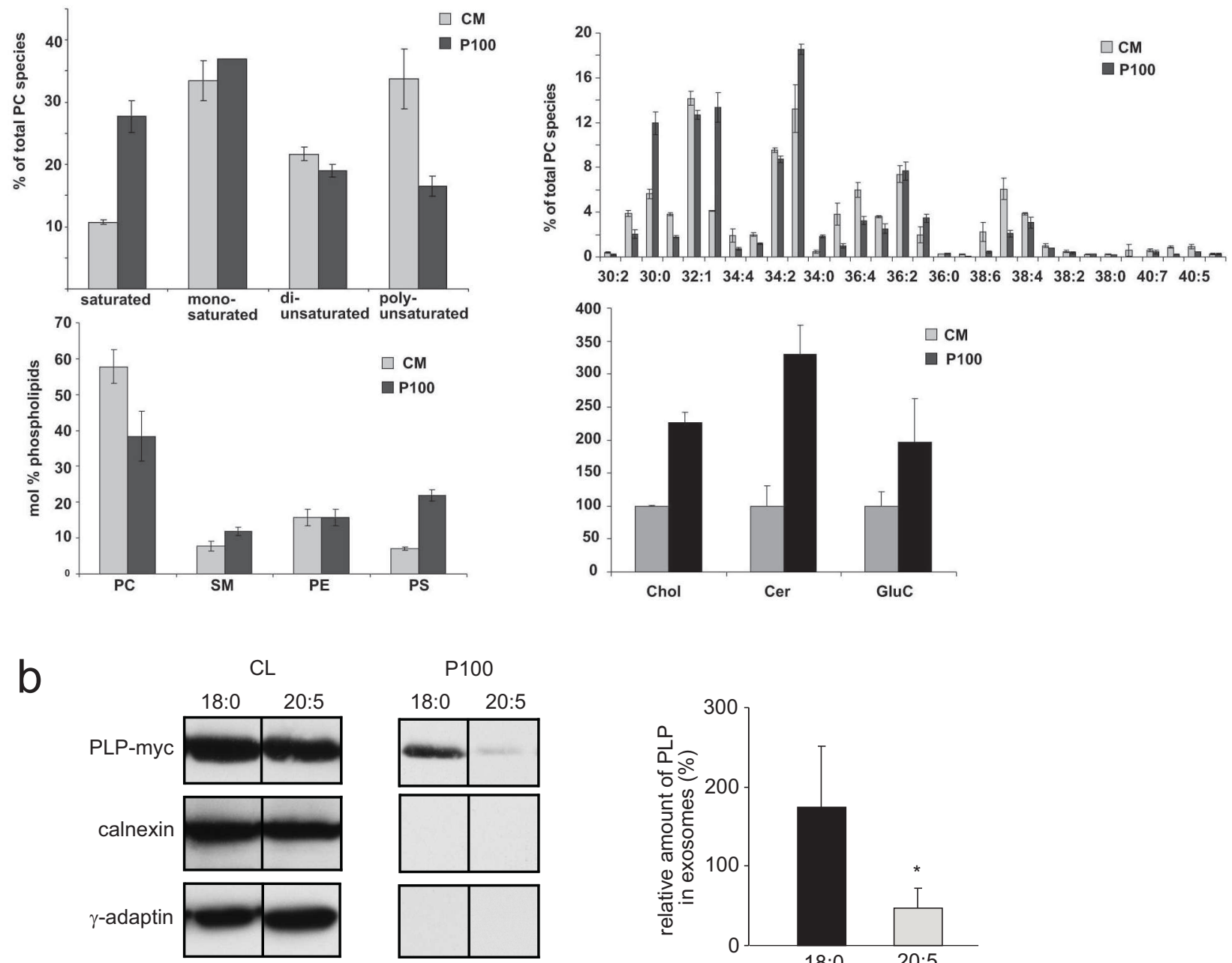

P100
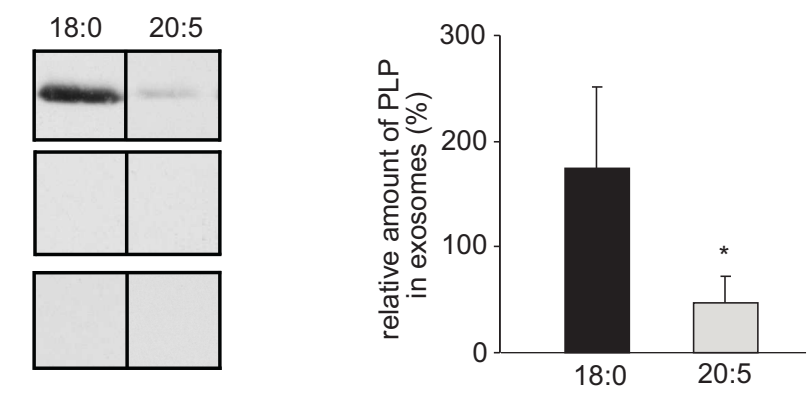

Figure 2 
a

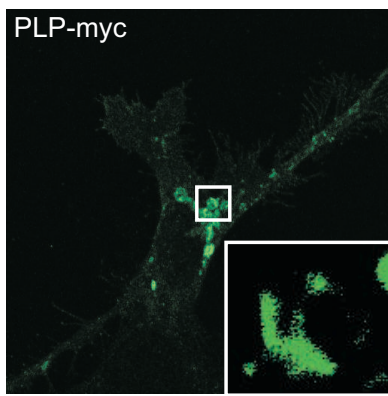

b

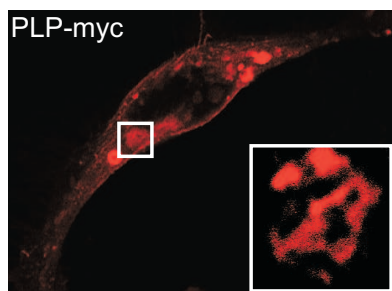

PLP-myc

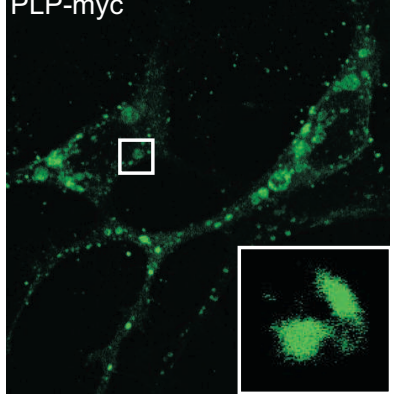

C
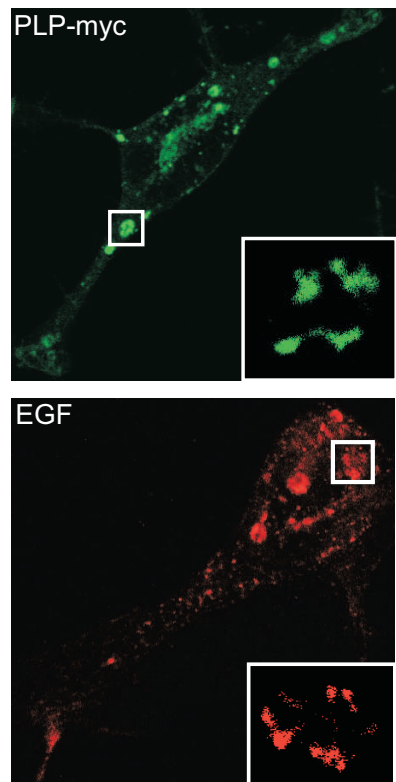

d

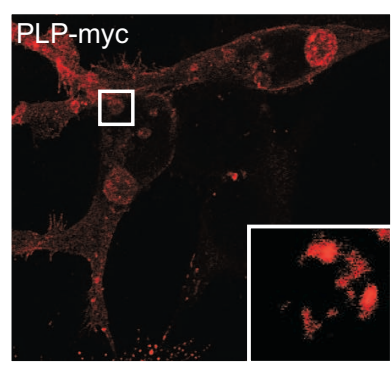

EGF

圆

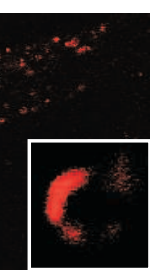

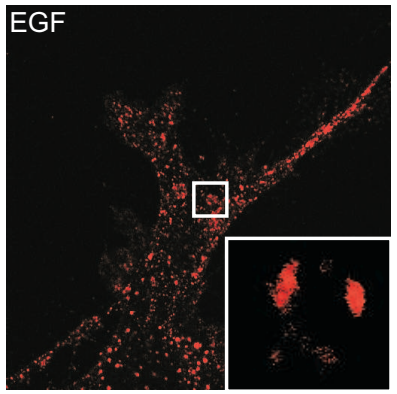
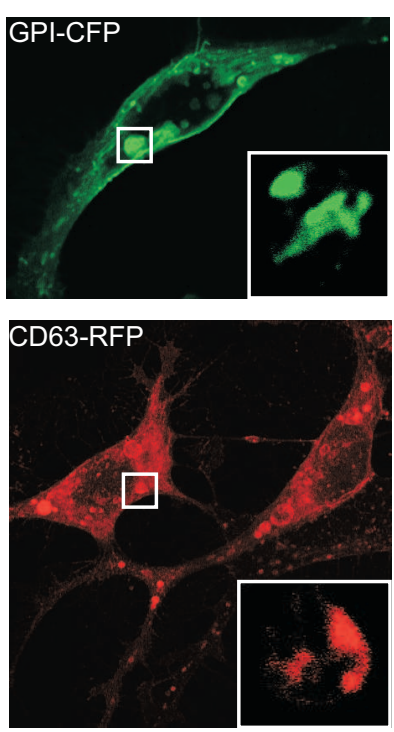

Flotillin-2-RFP
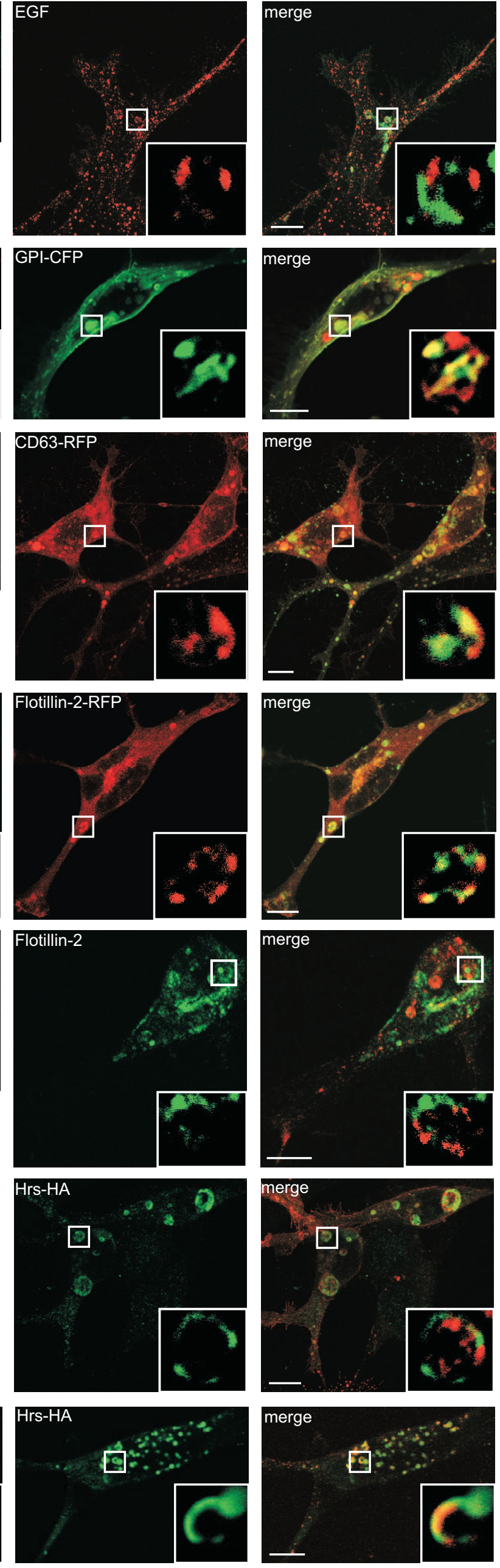

回.

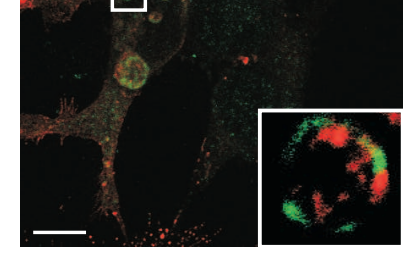

Figure 3 
a
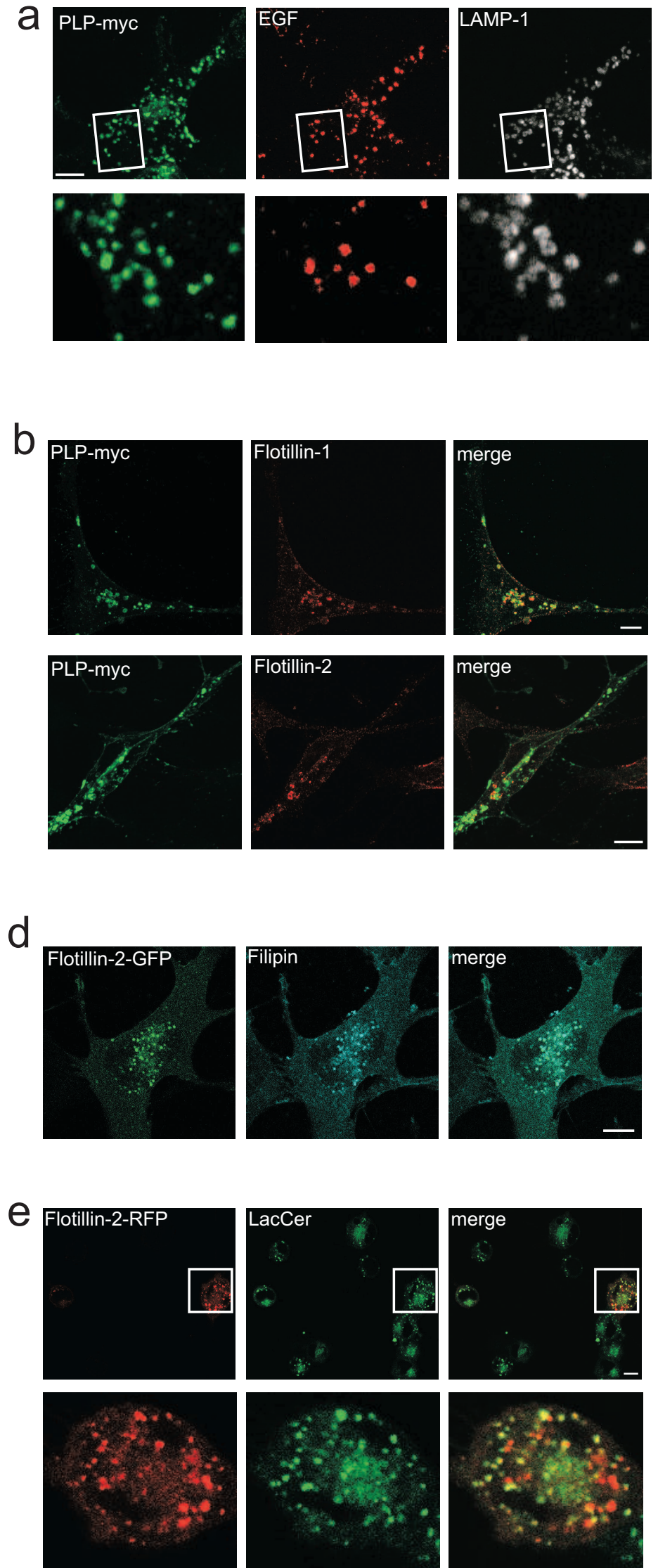
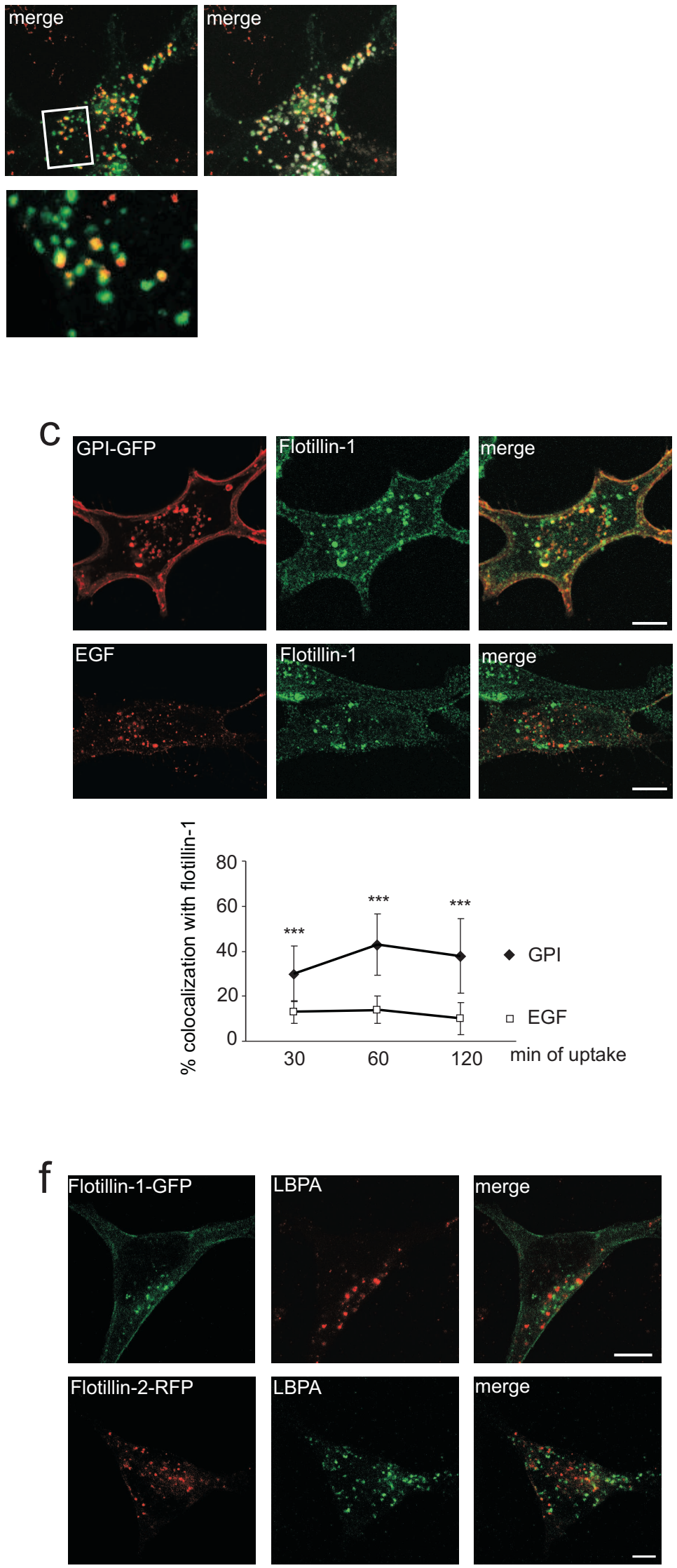

Figure 4 

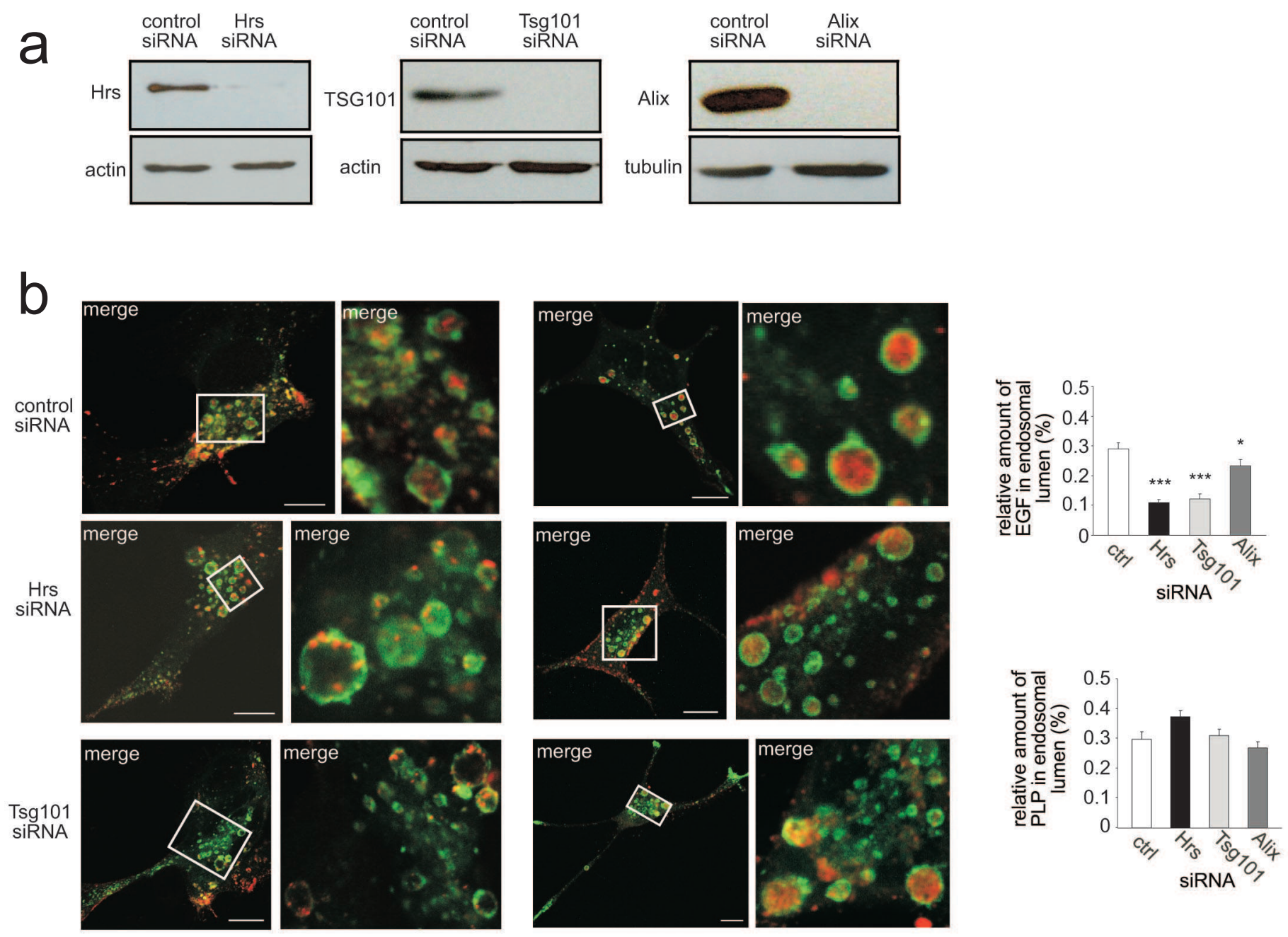

C
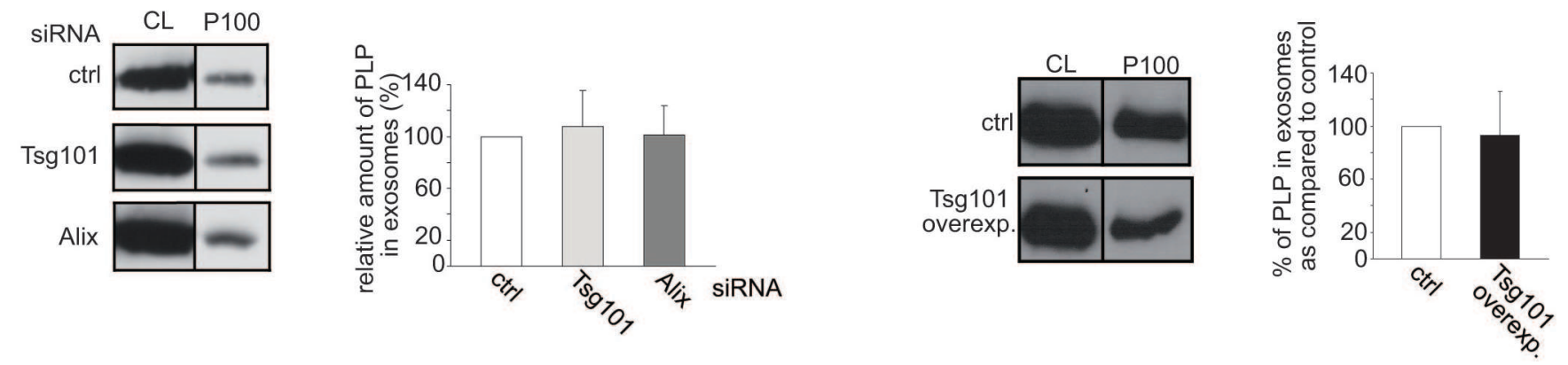

d
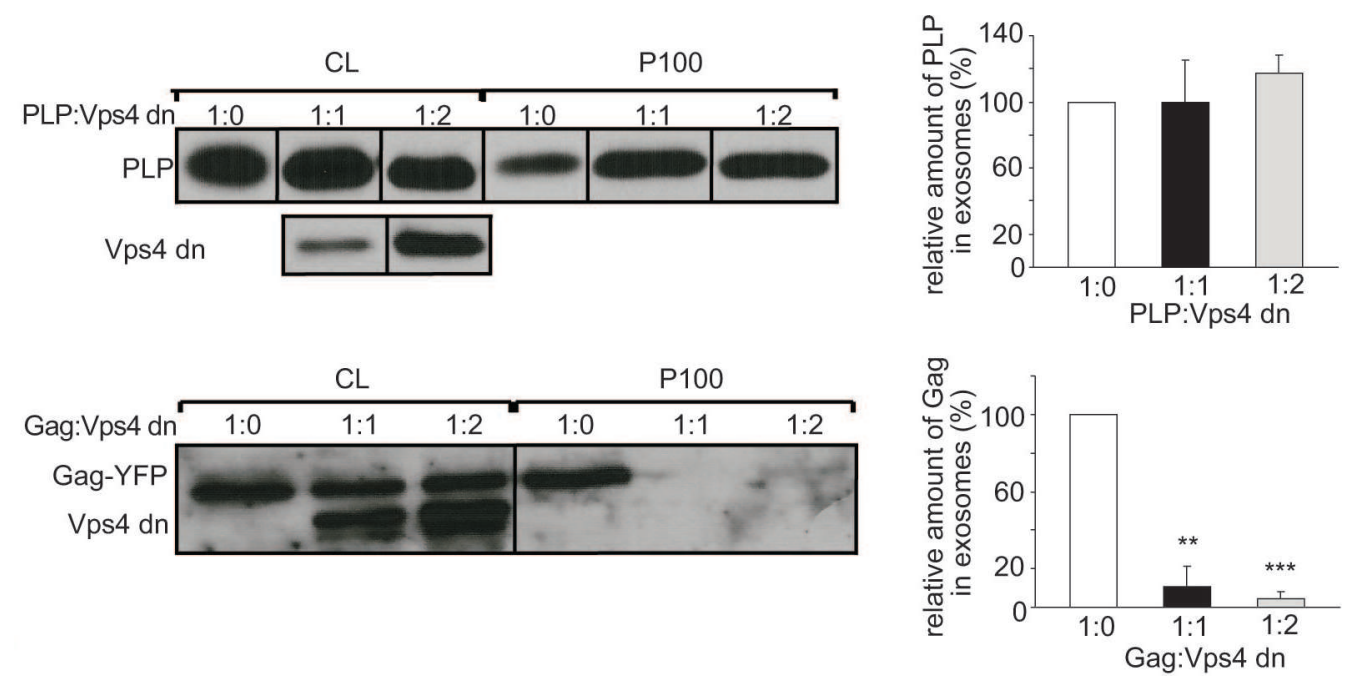


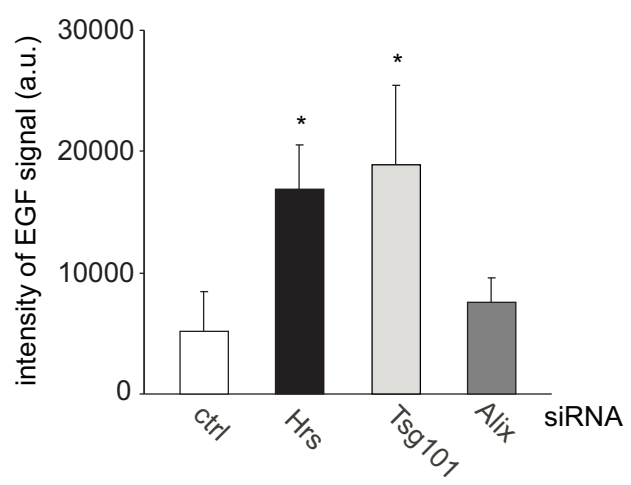

Figure S1 

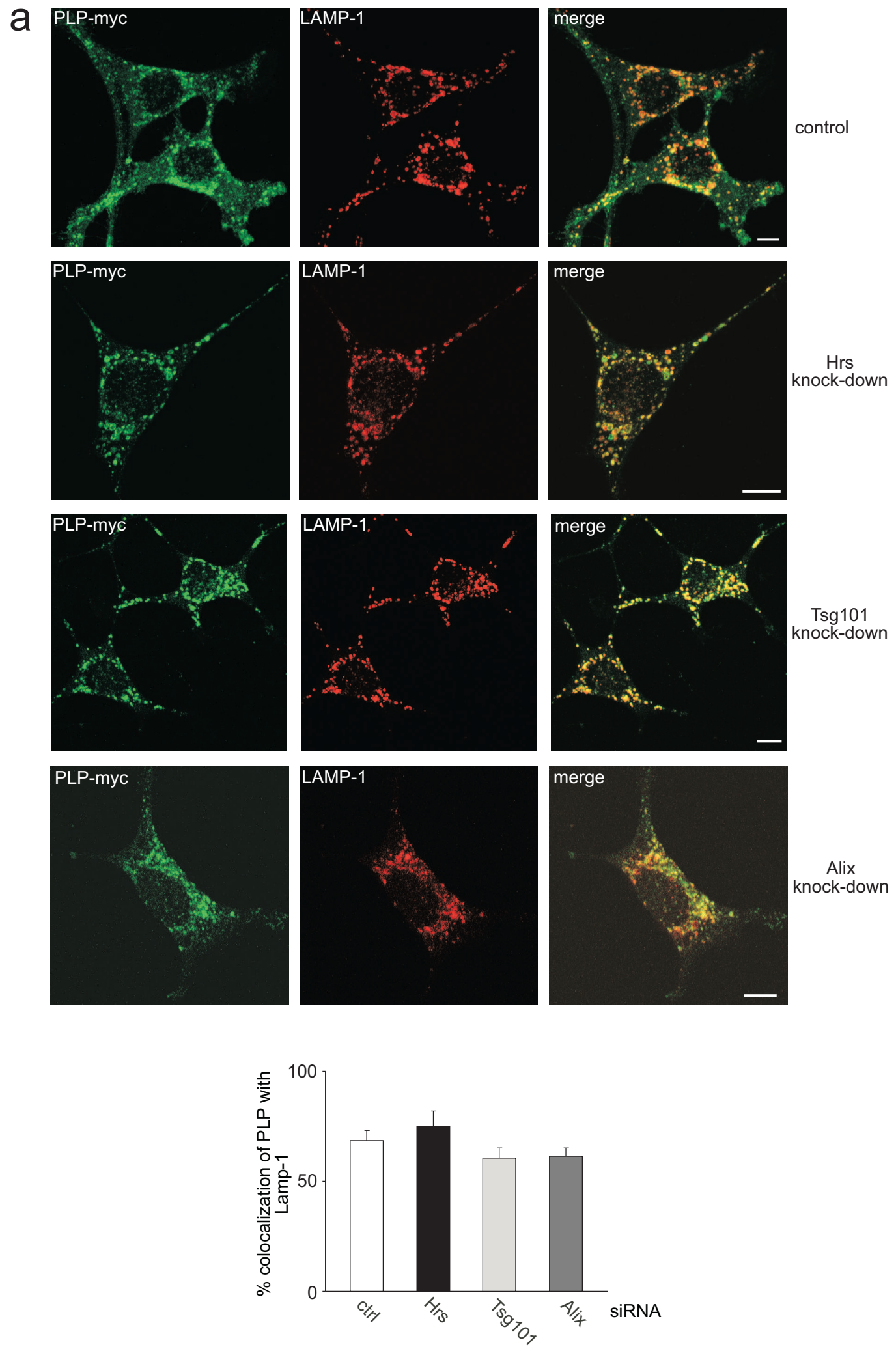

Figure S2 


\section{Discussion}

Our data demonstrate that oligodendrocytes use a clathrin-independent but cholesterol-depletion sensitive endocytosis pathway for the internalization of PLP, the major myelin membrane protein. Endocytosed PLP accumulates in the limiting membranes and ILVs of late endosomes. The sorting of PLP into the intralumenal membranes (ILMs) of multivesicular endosomes (MVEs) does not require the ESCRT-machinery. Upon arrival of soluble neuronal signals, clathrin-independent endocytosis is inhibited, PLP-enriched MVEs mobilized and delivered to the plasma membrane. This change in membrane trafficking is accompanied by the morphological differentiation of the cell, and regulated by changes in Rho GTPase activity. We propose that this change in myelin membrane trafficking is required to coordinate myelin membrane growth in the CNS.

\subsection{Regulation of endocytosis in oligodendrocytes}

We have identified a clathrin-independent, cholesterol-dependent endocytosis pathway used by PLP in oligodendroglial cell lines. Our conclusion was based on experiments using pharmacological and dominantnegative approaches. We found that the inhibition of clathrin-mediated endocytosis by the expression of dominant-negative mutant form of Eps 15 $(E \Delta 95 / 295)$ or potassium depletion did not interfere with the endocytosis of PLP. Instead, we observed a dramatic decrease of PLP internalization after depletion of cellular cholesterol under experimental conditions, which did not disturb clathrin-mediated endocytosis. Furthermore, we found that the endocytosis of PLP required the function of dynamin and the actin cytoskeleton. Finally, our results indicated a role of the GTPase RhoA in this pathway.

This endocytosis pathway could be related to a previosly described clathrin- and caveolin-independent uptake of GPI-anchored proteins and fluidphase in Cos-7 and chinese hamster ovary ( $\mathrm{CHO}$ ) cells (Sabharanjak et al., 
2002). In fact, we observed that a GPI-anchored GFP fusion protein and dextran, a fluid phase marker, used a clathrin-independent pathway in Oli-neu cells. However, we found that this pathway was regulated by RhoA and not as previously described, by cdc42. Therefore, this form of endocytosis may be more closely related to the RhoA regulated pathway used by IL-2 receptor (Lamaze et al., 2001) and the yc cytokine receptor (Sauvonnet et al., 2005). Future work is required to clearly define whether these different endocytosis pathways represent distinct portals of entry into a cell, or rather correspond to cell-type dependent variations of the same pathway. The fact that they all seem to be specialized to regulate the endocytosis of cholesterol and sphingolipids point to important paralleles. In the case of oligodendrocytes, we propose that this endocytosis pathway plays an important role in the regulation of the plasma membrane composition. The formation of myelin, which is highly enriched in cholesterol and specific sphingolipids may require the down-regulation of this pathway. This would lead to a gradual build-up of myelin-membrane components in the plasma membrane, given that clathrinmediated endocytosis, which does not show the same preference for cholesterol and sphingolipids is unchanged.

Indeed, we observed that the presence of neurons led to a remarkable inhibition of the endocytosis of PLP, but not of the clathrin-mediated endocytosis. Interestingly, we were able to show that the molecular mechanisms of this inhibition involved the regulation of RhoA. Thus, our data show that neurons control a specific endocytosis pathway in oligodendrocytes by regulating Rho GTPase activity.

We suggest that this regulation represents a mechanism to control myelin membrane growth at the appropriate time of development. It is an interesting example of how cell to cell signaling controls membrane trafficking to coordinate changes in cell architecture.

The exact nature of the neuronal signals and their mode of action will have to be addressed in future studies. 


\subsection{Sorting of proteolipid protein into multivesicular endosome}

After being endocytosed, PLP starts to accumulate in endosomes which either have a multivesicular or multivesicular/multilamellar appearance and contain Lamp-1. We refer to this organelle as late endosome (LE). PLP was found both on the limiting membrane and on the intralumenal membranes (ILMs) of this compartment. We found that sorting of PLP into ILMs did not require ESCRT-machinery. The conclusions were based on experiments studying the distribution of PLP on enlarged endosomes by use of the GTPase-deficient mutant form of Rab5 (Rab5 ${ }^{\mathrm{Q79L}}$ ) as a tool. Rab5 ${ }^{\mathrm{Q} 79 \mathrm{~L}}$ induces homo- and heterotypic fusion of endosomes (Stenmark et al., 1994) and leads to the formation of large endosomal compartment with ILMs clearly detectable by confocal microscopy. In such a system, we were able to follow the sorting of both PLP and the epidermal growth factor receptor (EGFR) into ILMs. As expected, we found that EGFR was sorted into ILMs via ESCRT-dependent pathway. We used siRNA and dominant-negative approaches to come to this conclusion. SiRNA-mediated downregulation of ESCRT-0 component Hrs strongly inhibited the sorting of EGFR into ILMs, but it did not affect sorting of PLP. Nevertheless, there are examples of proteins, such as the transferrin receptor, which can bind directly to ESCRT-I, bypassing the requirement for Hrs in sorting into endosomal lumen (Geminard et al., 2004). Human immunodeficiency virus (HIV) also does not demand Hrs for budding from the plasma membrane (Morita and Sundquist, 2004). However, the downregulation of Tsg101, the ESCRT-I component, did not disturb the targeting of PLP into the enlarged endosomes. Furthermore, the sorting of PLP was not significantly influenced by the interference with other proteins implicated in the ESCRT-pathway, such as Alix, clearly showing that sorting of PLP is an ESCRT-independent process. Together, these data suggest that the sorting of PLP into ILMs is accomplished through a pathway essentially different from previously described routes.

This conclusion was further supported by analyzing the microdomain structure of the Rab5 ${ }^{\text {Q79L }}$-enlarged endosome. We found that PLP did not colocalize with the previously described clathrin-coated microdomains, which act as platforms for the recruitment of ESCRT components (Raiborg et al., 
2001). In contrast to the EGFR, a well known example for the ESCRTdependent sorting, we found no colocalization of PLP with Hrs on the endosomes or with EGFR itself. Instead, we observed the colocalization of PLP with markers of lipid-rafts, GPI-anchored GFP and flotillin and with the tetraspanin protein, CD63. This result suggests that PLP might use rafts as a platform for inward budding, and it is consistent with previous observations that there is more than one type of ILMs in late endosomes (Mobius et al., 2003).

PLP-positive ILMs continued to form in cells in which the function of ESCRT was inhibited, implying that there are alternative pathways for sorting into the endosomal lumen. So far, one study has been able to identify the possible mechanisms of such a pathway (Matsuo et al., 2004). This study shows that the lipid lysobisphosphatidic acid (LBPA) was sufficient to drive the inward budding of membrane into an endosome. This mechanism seems to rely on the cone-shaped structure of the lipid, which promotes the formation of negative curvature of the membrane. Interestingly, Alix was involved in the control of this invagination process. Our finding that Alix depletion has no influence on the sorting of PLP into ILMs argues for an additional, distinct pathway of inward budding.

A recent study from Theos et al. (2006) provides evidence for the existence of a pathway for sorting into and formation of ILVs, which does not involve ESCRT, but requires luminal sorting determinant within the melanosomal protein Pmel.

There are also studies revealing that more than one population of MVEs exists within one cell (Mobius et al., 2003; White et al., 2006). One subset of MVEs is enriched in cholesterol, while others contain high levels of LBPA. Our results raise the question whether the differential sorting of PLP and EGFR and their segregation in ILMs lead to the targeting of these proteins to distinct MVEs. Both PLP and EGFR are targeted to Lamp-1 positive vesicles. However, we demonstrate that there is a subset of these vesicles which contain PLP, but not EGFR. The possibility that EGFR was not detected due to its rapid degradation in these vesicles seems unlikely since the treatment with the protease inhibitor leupeptin leads to similar results. The precise nature of this compartment remains to be established, but flotillin- 
enriched late endosomes are likely candidate organelles. PLP displays a significant colocalization with flotillin-positive endosomes, which also accumulate cholesterol and the glycosphingolipid BODIPY-lactosylceramide, but do not contain detecable levels of LBPA. These data suggest that the previously described cholesterol-rich MVEs are a subpopulation of late endosomes defined by the presence of flotillin.

How does the sorting of PLP occur? The fact that PLP colocalizes with lipid-raft proteins in ILMs and associates with cholesterol- and sphingolipidenriched MVEs indicates that PLP might segregate using lipid rafts as a sorting mechanism. Further studies will have to address this question in more detail.

\subsection{Retrograde membrane transport of PLP}

Our data demonstrate that the treatment of Oli-neu cells with conditioned neuronal medium or inactivation of Rho leads to a dramatic change in the distribution of PLP. Namely, the colocalization of PLP and Lamp-1 was lost and PLP appeared instead predominantly on the plasma membrane. This redistribution was observed both in oligodendroglial cell line and in primary cultures of oligodendrocytes. Importantly, the same switch in localization was detected in situ when brain sections from young (postnatal day 7, P7) and old (P60) mice were compared. Colocalizaton of PLP and Lamp-1 was only observed in early developmental steps, P7, but not when myelination was completed, in P60.

What happens with PLP after receiving of the neuronal signals? Our data obtained from pulse-chase experiments showed that the degradation rate of PLP occured at similar rates in the presence and absence of neurons. Therefore, the possibility that neuronal factors induced the degradation of PLP seems unlikely. One way to explain this change in PLP localization was already discussed previously and involves the inhibition of endocytosis by neuronal factors. Another possibility is that retrograde transport from late endosomes to the plasma membrane is induced by neurons. 
PLP-enriched MVEs are relatively stationary vesicles clustered in the perinuclear region. Conversely, due to the arrival of neuronal signals and the expression of dominant-negative mutant form of RhoB GTPase, at least one subset of these vesicles becomes highly mobile and can be detected in the peripheral area of the cell. The same effect was achieved by inhibition of tyrosin kinases. Expression of constitutively active mutant form of RhoB GTPase and stimulation of Src kinase abolished the effect of neuronal factors on the mobility of MVEs. These results are consistent with previous studies showing that $\mathrm{RhoB}$ regulates the dynamics of endosomes (Fernandez-Borja et al., 2005) and Src activity (Sandilands et al., 2004).

Indeed, by using total internal reflection fluorescence microscopy (TIRFM), we show that PLP-containing acidic endosomes have the ability to fuse with the plasma membrane in Oli-neu cells treated with neuronal medium. The extensive colocalization of PLP with LysoTracker, a marker for a low-pH environment, suggests that PLP might be delivered to the cell surface by being associated with these acidic compartments. These data raise the possibility that neuronal signals regulate not only the mobility, but also the fusion of late endosomes with the plasma membrane. It remains to be analyzed whether inhibition of RhoB and Src increases the frequency of fusion events.

The transport of PLP in Oli-neu cells is strikingly similar to the trafficking of the major histocompatibility complex class II (MHC-II) in dendritic cells (Trombetta and Mellman, 2005). Immature dendritic cells store internalized $\mathrm{MHC}-\mathrm{II}$ in late endomal compartment with a multivesicular appearance which is refered to as MHC class II compartment (MIIC). Upon maturation, the retrograde transport is triggered and $\mathrm{MHC}-\mathrm{II}$ is delivered to the plasma membrane. The transport of PLP from MVEs could also be related to the regulated exocytosis of secretory lysosomes in hematopoietic cells.

The PLP-enriched late endosome does not seem to be a terminal degradative organelle, as suggested by the absence of the electron-dense core typical for lysosomes. However, degradation can also take place in prelysosomal compartment due to its acidic environment and the presence of proteolytic enzymes. The degradation of PLP is not likely to occur in these organelles, as they can accumulate PLP and deliver it to the cells surface in a 
regulated fashion. In addition, recent data have shown that the degradation of PLP involves proteasomal function (Kramer-Albers et al., 2006; McLaughlin et al., 2006). One possible explanation for the stability of PLP in this compartment is that this protein is poorly degradable. For example, constitutive lysosomal residents LAMPs and LIMPs are resistant to degradative environment due to their post-translational modifications, asparagine-linked glycans (Kundra and Kornfeld, 1999). Another possibility is that these late endosomes are poor in proteolytic enzymes, as in the case of late endosomes/lysosomes (LE/Ls) that store MHC-II in immature dendritic cells and that have reduced degradative properties (Trombetta et al., 2003). However, if PLP is, indeed, transported back to the plasma membrane, an escape mechanism from the LEs must exist. The fraction of PLP found on the limiting membrane of LE could incorporate into the plasma membrane directly, after the fusion of these organelles, whereas the fraction of PLP within the ILMs must take an additional step, such as back-fusion of ILMs with the limiting membrane. There are several examples of proteins which can exit from the ILVs. Maturation of dendritic cells can trigger back-fusion of MHC-IIenriched ILVs with the limiting membrane of LE/Ls. This process is accompanied by the formation of the tubular extensions on LE/Ls (Trombetta and Mellman, 2005). Tubules derived from LE/L eventually fuse with the plasma membrane, leading to a massive transport of MHC-II to the cell surface without a concomitant release of proteolitic enzymes into the extracellular milieu. Similar back-fusion has been observed for the antrax toxin subunits edema factor and lethal factor (Abrami et al., 2004), or for the vesicular stomatitis virus (Le Blanc et al., 2005). These viral subunits rely on back-fusion of ILVs with the limiting membrane of a late endosome as a mechanism of their release into the cytoplasm. It remains to be determined whether PLP is sorted to the cell surface by back-fusion and tubules in response to neuronal signals.

In addition to the sorting of PLP to the plasma membrane, we found that this protein can follow another pathway. It can be released into the extracellular environment in association with exosomes. These microvesicles are secreted upon fusion of MVEs with the plasma membrane. The physiological function of this pathway is unknown. Exosomes could represent 
a vehicle for exporting of superfluous membrane from the inside of the cell to the extracellular milieu.

\subsection{Conclusions}

Our findings shed some light on the regulation of membrane transport in oligodendrocytes. In the absence of neuronal factors, there is a high level of clathrin-independent endocytosis, followed by the targeting of endocytosed material to LE. Upon arrival of neuronal signals, which is accompanied by the morphological differentiation of the cells, initiation of the retrograde membrane transport occurs synchronously with the inhibition of clathrin-independent endocytosis. Neurons may induce a switch between these two modes of membrane trafficking.

It is interesting to compare the regulation of PLP trafficking in oligodendrocytes by neuronal signals with the changes in membrane trafficking during the development of polarized tissue (Mostov, 2003). During tubulogenesis, cells depolarize and develop intracellular compartments which contain components that are normally found on the surface. Polarization, by contrast, often involves the redirection of membrane from intracellular reservoirs to the plasma membrane. This is one example of how membrane trafficking can undergo profound changes during development.

The switch between two modes of membrane trafficking may have an important role in myelin biogenesis. The formation of myelin requires fast and timely delivery of huge amounts of myelin-specific lipids and proteins. The switch between endocytosis and retrograde transport could lead to the enrichment of the plasma membrane in myelin constituents. A specific form of cholesterol/sphingolipid-dependent endocytosis that we have identified in oligodendrocytes might lead to the intracellular accumulation of these lipids together with the major myelin protein PLP. Conversely, the reduction of this specific form of endocytosis may lead to a gradual deposition of myelin lipids and PLP within the plasma membrane of oligodendrocytes. The retrograde transport and exocytosis of previously internalized myelin-specific constituents could support the qualitative change of the plasma membrane in a rapid 
manner. Indeed, our experimental data confirm the idea that the composition of the plasma membrane changes during the morphological differentiation. Laurdan staining, a way to measure membrane condensation, has shown a significant increase in the lipid order of the plasma membrane upon arrival of neuronal signals or Rho-inhibition. Recent study from Fitzner et al. (2006) documents that the process of myelination is associated with a condensation of plasma membrane in neuron-oligodendrocyte cocultures.

Oligodendrocytes also regulate the size of their plasma membrane during development. The surface of oligodendrocytes becomes eventually approximately 10000 times larger as compared with that of a typical animal cell (Pfeiffer et al., 1993). Decreased endocytosis and increased exocytosis could result in the extension of the cell surface. After differentiation, oligodendrocytes may shift intracellular trafficking towards a high rate of exocytosis and a low rate of endocytosis to extend their plasma membrane. An increased influx directly from the biosynthetic pathway is also likely to contribute to this process.

Endocytosis and retrograde transport in Oli-neu cells are regulated by members of the same Rho GTPase family. The activity of Rho GTPases may act as a switch between these two modes of membrane trafficking. Rho GTPases control many features of cell behaviour, including profound effects on the organization of actin cytoskeleton (Ridley and Hall, 1992) and vesicular trafficking (Gulli and Peter, 2001; Symons and Rusk, 2003; Symons and Settleman, 2000). Deeper insight into the regulation of membrane trafficking by Rho family GTPases comes from the fact that different family members display diverse distribution in the cell, which indicates distinct functions for each of them (Ridley, 2006). RhoA is mainly cytosolic and it acts at an early step of the internalization process (also shown by Lamaze et al., (1996), Vogler et al., (1999) and Hrboticky et al., (2002)). RhoB localizes both to the plasma membrane and to endosomes, and it can control endosomal dynamics through the Src kinase (Sandilands et al., 2004). Moreover, RhoDSrc signaling cascade regulates the dynamics of early endosomes (Gasman et al., 2003). These studies, together with our results, suggest that different Rho GTPases could be involved in multiple steps of the endocytic pathway. 
Our result that Rho inhibition is important for oligodendrocyte differentiation is consistent with other studies. One pathway recently shown to coordinate myelination is the regulation of RhoA activity by LINGO-1 in oligodendrocytes (Mi et al., 2005). Activation of RhoA by LINGO-1 negatively regulates myelination (Liang et al., 2004; Mi et al., 2005). In the light of our data, one possible mechanism of action for this signaling pathway could be the regulation of the cellular machinery involved in myelin membrane trafficking.

Neurons might control the differentiation of oligodendrocytes by regulating Rho activity. Interestingly, oligodendrocytes secrete myelin-based inhibitory signals which can restrict axonal regeneration by activating RhoA signaling in neurons (Yiu and $\mathrm{He}, 2006$ ). Thus, it seems that neurons and oligodendrocytes use Rho signaling cascade for bidirectional communication.

Importantly, not all myelin components use the same trafficking pathway. In contrast to PLP and cholesterol which reside in MVEs, myelin basic protein and galactosylceramide are mainly found on the plasma membrane (Fitzner et al., 2006). The differential distribution of myelin constituents before the neuronal stimulation and the onset of myelination might be a mechanism to prevent their premature and irregular assembly. Exosomes could be utilized for the elimination of excessive material from the storage compartment before it can be incorporated into myelin.

Together, our findings reveal that a clathrin-independent endocytosis leads to the delivery of endocytosed content into the endosomal compartment with a unique and unexpected function - to store and release its content in a regulated manner. This process may have an essential role in myelin biogenesis. The regulation of transport to and from this compartment by soluble neuronal signals is an astonishing example of how intercellular communication can guide the development of multicellular tissue through the control of intracellular trafficking. 


\section{Appendix}

\subsection{Abbreviations}

${ }^{\circ} \mathrm{C}$

a.u.

$A b$

AP-2

ARF6

BSA

ca

CAMP

CDNA

CFP

$\mathrm{CHO}$

ChxB

C.L.

CLIC

CNP

CNS

CNTF

ctrl

d

DIV

DMEM

DMSO

dn

dyn

ECV/MVB

EGF

EGFR

EGFP

EM

eps 15

ER

ESCRT

EYFP degree centigrade

arbitrary unit

antibody

adaptor protein 2

ADP-ribosylation factor 6

bovine serum albumine

constitutively active

cyclic 3'-5'-cyclic adenosine monophosphate

complementary deoxiribonucleic acid

cyan fluorescent protein

chinese hamster ovary

B subunit of cholera toxin

cell lysate

clathrin-independent carrier

2',3'-cyclic nucleotide-3'-phosphohydrolase

central nervous system

ciliary neurotrophic factor

control

day

days in vitro

Dulbecco's modified Eagle's medium

dimethylsolfoxide

dominant-negative

dynamin

endosomal carrier vesicle/multivesicular body

epidermal growth factor

EGF receptor

enhanced green fluorescent protein

electron microscopy

epidermal growth factor receptor pathway substrate 15

endoplasmic reticulum

endosomal sorting complex required for transport

enhanced yellow fluorescent protein 


\begin{tabular}{|c|c|}
\hline FB1 & fumonisin B1 \\
\hline FCS & fetal calf serum \\
\hline FGF-2 & fibroblast growth factor-2 \\
\hline FITC & fluorescein \\
\hline FRET & fluorescence resonance energy transfer \\
\hline GalC & galactosylceramide \\
\hline GEEC & GPI-enriched endosomal compartment \\
\hline GFP & green fluorescent protein \\
\hline GP & generalized polarization \\
\hline GPI & glycosylphosphatidylinositol \\
\hline GST & glutathione S-transferase \\
\hline GTP & guanosine triphosphate \\
\hline $\mathrm{h}$ & hour \\
\hline HA & hemagglutinin \\
\hline HBSS & Hanks' balanced salt solution \\
\hline HEPES & N-2-hydroxyethylpiperazin-N'-2-ethansulfonic acid \\
\hline HRP & horseradish peroxidase \\
\hline Hrs & hepatocyte growth factor-regulated tyrosine kinase substrate \\
\hline HS & horse serum \\
\hline $\lg$ & immunoglobulin \\
\hline IGF-1 & insulin-like growth factor 1 \\
\hline IL-2 & interleukin receptor 2 \\
\hline ILM & intralumenal membrane \\
\hline ILV & intralumenal vesicle \\
\hline $\mathrm{kDa}$ & kilo Dalton \\
\hline LacCer & lactosylceramide \\
\hline Lamp & lysosome-associated membrane protein \\
\hline LBPA & lysobisphosphatidic acid \\
\hline LE & late endosome \\
\hline LE/L & late endosome/lysosome \\
\hline LIF & leukemia inhibitory factor \\
\hline Limp & lysosomal integral membrane protein \\
\hline LysoT & LysoTracker \\
\hline M6PR & mannose 6-phosphate receptor \\
\hline $\mathrm{mAb}$ & monoclonal antibody \\
\hline MAG & myelin-associated glycoprotein \\
\hline MBP & myelin basic protein \\
\hline MEM & minimum essential medium \\
\hline $\mathrm{MHC}$ & major histocompatibily complex \\
\hline MIIC & MHC class II compartment \\
\hline
\end{tabular}




\begin{tabular}{|c|c|}
\hline $\min$ & minute \\
\hline MOG & myelin/oligodendrocyte glycoprotein \\
\hline mRNA & messenger ribonucleic acid \\
\hline MVE & multivesicular endosome \\
\hline MW & molecular weight \\
\hline$m \beta C D$ & methyl- $\beta$-cyclodextrin \\
\hline NCAM & neural cell adhesion molecule \\
\hline$N R G$ & neuregulin \\
\hline NT-3 & neurotrophin-3 \\
\hline NZF & Npl4 zinc finger \\
\hline $\mathrm{o} / \mathrm{n}$ & over night \\
\hline OPC & oligodendrocyte precursor cell \\
\hline OSP & oligodendrocyte-specific protein \\
\hline $\mathrm{P}$ & postnatal day \\
\hline PBS & phosphate-buffered saline \\
\hline PDGF-A & plateled-derived growth factor $A$ \\
\hline PDGFR- $\alpha$ & plateled-derived growth factor $\alpha$-receptor \\
\hline PFA & paraformaldehyde \\
\hline $\mathrm{PI} 3 \mathrm{P}$ & phosphatidylinositol-3-phosphate \\
\hline PKN & protein kinase $\mathrm{N}$ \\
\hline PLL & poly-L-lysine \\
\hline PLP & proteolipid protein \\
\hline PMP22 & peripheral myelin protein 22 \\
\hline PNS & peripheral nervous system \\
\hline $\mathrm{pOL}$ & primary oligodendrocytes \\
\hline $\operatorname{PrP}^{c}$ & prion protein \\
\hline Ptdlns $(4,5) \mathrm{P}_{2}$ & phosphatidylinositol 4,5-bisphosphate \\
\hline RT & room temperature \\
\hline SD & standard deviation \\
\hline SDS-PAGE & sodium dodecylsulfate polyacrylamide gel electrophoresis \\
\hline SNARE & soluble $\mathrm{N}$-ethylmaleimide-sensitive factor attachment protein receptor \\
\hline StAR & steroidogenic acute regulatory protein \\
\hline SV40 & Simian Virus 40 \\
\hline Tf & transferrin \\
\hline TGF- $\beta$ & transforming growth factor $\beta$ \\
\hline TIRFM & total internal reflection fluorescence microscopy \\
\hline Tsg101 & tumor susceptibility gene 101 \\
\hline UIM & ubiquitine-interacting motif \\
\hline UTR & untranslated region \\
\hline Vps & vacuolar sorting protein \\
\hline
\end{tabular}


VSV

WB

wt

vesicular stomatitis virus

western blotting

wild-type 


\section{References}

Abrami, L., Lindsay, M., Parton, R. G., Leppla, S. H., and van der Goot, F. G. (2004). Membrane insertion of anthrax protective antigen and cytoplasmic delivery of lethal factor occur at different stages of the endocytic pathway. J Cell Biol 166, 645-651.

Abrami, L., Liu, S., Cosson, P., Leppla, S. H., and van der Goot, F. G. (2003). Anthrax toxin triggers endocytosis of its receptor via a lipid raft-mediated clathrin-dependent process. J Cell Biol 160, 321-328.

Ainger, K., Avossa, D., Diana, A. S., Barry, C., Barbarese, E., and Carson, J. H. (1997). Transport and localization elements in myelin basic protein mRNA. J Cell Biol 138, 10771087.

Ainger, K., Avossa, D., Morgan, F., Hill, S. J., Barry, C., Barbarese, E., and Carson, J. H. (1993). Transport and localization of exogenous myelin basic protein mRNA microinjected into oligodendrocytes. J Cell Biol 123, 431-441.

Alam, S. L., Sun, J., Payne, M., Welch, B. D., Blake, B. K., Davis, D. R., Meyer, H. H., Emr, S. D., and Sundquist, W. I. (2004). Ubiquitin interactions of NZF zinc fingers. Embo J 23, 1411-1421.

Alpy, F., Stoeckel, M. E., Dierich, A., Escola, J. M., Wendling, C., Chenard, M. P., Vanier, M. T., Gruenberg, J., Tomasetto, C., and Rio, M. C. (2001). The steroidogenic acute regulatory protein homolog MLN64, a late endosomal cholesterol-binding protein. J Biol Chem 276, 4261-4269.

Amerik, A. Y., Nowak, J., Swaminathan, S., and Hochstrasser, M. (2000). The Doa4 deubiquitinating enzyme is functionally linked to the vacuolar protein-sorting and endocytic pathways. Mol Biol Cell 11, 3365-3380.

Andrews, N. W. (2000). Regulated secretion of conventional lysosomes. Trends Cell Biol 10, 316-321.

Andrews, N. W. (2002). Lysosomes and the plasma membrane: trypanosomes reveal a secret relationship. J Cell Biol 158, 389-94.

Aniento, F., Emans, N., Griffiths, G., and Gruenberg, J. (1993). Cytoplasmic dyneindependent vesicular transport from early to late endosomes. J Cell Biol 123, 1373-1387.

Babst, M. (2005). A protein's final ESCRT. Traffic 6, 2-9.

Babst, M., Katzmann, D. J., Estepa-Sabal, E. J., Meerloo, T., and Emr, S. D. (2002a). Escrt-III: an endosome-associated heterooligomeric protein complex required for mvb sorting. Dev Cell 3, 271-282.

Babst, M., Katzmann, D. J., Snyder, W. B., Wendland, B., and Emr, S. D. (2002b). Endosome-associated complex, ESCRT-II, recruits transport machinery for protein sorting at the multivesicular body. Dev Cell 3, 283-289.

Babst, M., Sato, T. K., Banta, L. M., and Emr, S. D. (1997). Endosomal transport function in yeast requires a novel AAA-type ATPase, Vps4p. Embo J 16, 1820-1831. 
Babst, M., Wendland, B., Estepa, E. J., and Emr, S. D. (1998). The Vps4p AAA ATPase regulates membrane association of a Vps protein complex required for normal endosome function. Embo J 17, 2982-2993.

Bache, K. G., Brech, A., Mehlum, A., and Stenmark, H. (2003). Hrs regulates multivesicular body formation via ESCRT recruitment to endosomes. J Cell Biol 162, 435-442.

Bansal, R., Warrington, A. E., Gard, A. L., Ranscht, B., and Pfeiffer, S. E. (1989). Multiple and novel specificities of monoclonal antibodies $\mathrm{O} 1, \mathrm{O} 4$, and $\mathrm{R}-\mathrm{mAb}$ used in the analysis of oligodendrocyte development. J Neurosci Res 24, 548-557.

Barbero, P., Bittova, L., and Pfeffer, S. R. (2002). Visualization of Rab9-mediated vesicle transport from endosomes to the trans-Golgi in living cells. J Cell Biol 156, 511-518.

Baron, W., Colognato, H., and ffrench-Constant, C. (2005). Integrin-growth factor interactions as regulators of oligodendroglial development and function. Glia 49, 467-479.

Barres, B. A., and Raff, M. C. (1994). Control of oligodendrocyte number in the developing rat optic nerve. Neuron 12, 935-942.

Baumann, N., and Pham-Dinh, D. (2001). Biology of oligodendrocyte and myelin in the mammalian central nervous system. Physiol Rev 81, 871-927.

Bilodeau, P. S., Urbanowski, J. L., Winistorfer, S. C., and Piper, R. C. (2002). The Vps27p Hse1p complex binds ubiquitin and mediates endosomal protein sorting. Nat Cell Biol 4, 534539.

Blott, E. J., and Griffiths, G. M. (2002). Secretory lysosomes. Nat Rev Mol Cell Biol 3, $122-$ 131.

Boes, M., Cerny, J., Massol, R., Op den Brouw, M., Kirchhausen, T., Chen, J., and Ploegh, H. L. (2002). T-cell engagement of dendritic cells rapidly rearranges MHC class II transport. Nature 418, 983-988.

Bomsel, M., Parton, R., Kuznetsov, S. A., Schroer, T. A., and Gruenberg, J. (1990). Microtubule- and motor-dependent fusion in vitro between apical and basolateral endocytic vesicles from MDCK cells. Cell 62, 719-731.

Bright, N. A., Gratian, M. J., and Luzio, J. P. (2005). Endocytic delivery to lysosomes mediated by concurrent fusion and kissing events in living cells. Curr Biol 15, 360-365.

Bunge, M. B., Bunge, R. P., and Pappas, G. D. (1962). Electron microscopic demonstration of connections between glia and myelin sheaths in the developing mammalian central nervous system. J Cell Biol 12, 448-453.

Bunge, R. P. (1968). Glial cells and the central myelin sheath. Physiol Rev 48, 197-251.

Burkhardt, J. K., Mcllvain, J. M., Jr., Sheetz, M. P., and Argon, Y. (1993). Lytic granules from cytotoxic $T$ cells exhibit kinesin-dependent motility on microtubules in vitro. J Cell Sci 104 ( Pt 1), 151-162.

Butt, A. M., and Ransom, B. R. (1989). Visualization of oligodendrocytes and astrocytes in the intact rat optic nerve by intracellular injection of lucifer yellow and horseradish peroxidase. Glia 2, 470-475. 
Canoll, P. D., Kraemer, R., Teng, K. K., Marchionni, M. A., and Salzer, J. L. (1999). GGF/neuregulin induces a phenotypic reversion of oligodendrocytes. Mol Cell Neurosci 13, 79-94.

Canoll, P. D., Musacchio, J. M., Hardy, R., Reynolds, R., Marchionni, M. A., and Salzer, J. L. (1996). GGF/neuregulin is a neuronal signal that promotes the proliferation and survival and inhibits the differentiation of oligodendrocyte progenitors. Neuron 17, 229-243.

Carlton, J. G., Bujny, M. V., Peter, B. J., Oorschot, V. M., Rutherford, A., Arkell, R. S., Klumperman, J., McMahon, H. T., and Cullen, P. J. (2005). Sorting nexin-2 is associated with tubular elements of the early endosome, but is not essential for retromer-mediated endosome-to-TGN transport. J Cell Sci 118, 4527-4539.

Carteron, C., Ferrer-Montiel, A., and Cabedo, H. (2006). Characterization of a neuralspecific splicing form of the human neuregulin 3 gene involved in oligodendrocyte survival. $J$ Cell Sci 119, 898-909.

Charles, P., Hernandez, M. P., Stankoff, B., Aigrot, M. S., Colin, C., Rougon, G., Zalc, B., and Lubetzki, C. (2000). Negative regulation of central nervous system myelination by polysialylated-neural cell adhesion molecule. Proc Natl Acad Sci U S A 97, 7585-7590.

Chow, A., Toomre, D., Garrett, W., and Mellman, I. (2002). Dendritic cell maturation triggers retrograde $\mathrm{MHC}$ class II transport from lysosomes to the plasma membrane. Nature 418, 988-994.

Coetzee, T., Suzuki, K., Nave, K. A., and Popko, B. (1999). Myelination in the absence of galactolipids and proteolipid proteins. Mol Cell Neurosci 14, 41-51.

Colman, D. R., Kreibich, G., Frey, A. B., and Sabatini, D. D. (1982). Synthesis and incorporation of myelin polypeptides into CNS myelin. J Cell Biol 95, 598-608.

Coman, I., Barbin, G., Charles, P., Zalc, B., and Lubetzki, C. (2005). Axonal signals in central nervous system myelination, demyelination and remyelination. J Neurol Sci 233, 6771.

Damm, E. M., Pelkmans, L., Kartenbeck, J., Mezzacasa, A., Kurzchalia, T., and Helenius, A. (2005). Clathrin- and caveolin-1-independent endocytosis: entry of simian virus 40 into cells devoid of caveolae. J Cell Biol 168, 477-488.

Deinhardt, K., Berninghausen, O., Willison, H. J., Hopkins, C. R., and Schiavo, G. (2006). Tetanus toxin is internalized by a sequential clathrin-dependent mechanism initiated within lipid microdomains and independent of epsin1. J Cell Biol 174, 459-471.

Di Guglielmo, G. M., Le Roy, C., Goodfellow, A. F., and Wrana, J. L. (2003). Distinct endocytic pathways regulate TGF-beta receptor signalling and turnover. Nat Cell Biol 5, 410421.

Doetsch, F., Garcia-Verdugo, J. M., and Alvarez-Buylla, A. (1997). Cellular composition and three-dimensional organization of the subventricular germinal zone in the adult mammalian brain. J Neurosci 17, 5046-5061.

Dubois-Dalcq, M., Behar, T., Hudson, L., and Lazzarini, R. A. (1986). Emergence of three myelin proteins in oligodendrocytes cultured without neurons. J Cell Biol 102, 384-392. 
Epple, U. D., Eskelinen, E. L., and Thumm, M. (2003). Intravacuolar membrane lysis in Saccharomyces cerevisiae. Does vacuolar targeting of Cvt17/Aut5p affect its function? J Biol Chem 278, 7810-7821.

Escola, J. M., Kleijmeer, M. J., Stoorvogel, W., Griffith, J. M., Yoshie, O., and Geuze, H. J. (1998). Selective enrichment of tetraspan proteins on the internal vesicles of multivesicular endosomes and on exosomes secreted by human B-lymphocytes. J Biol Chem 273, 20121 20127.

Fernandez, P. A., Tang, D. G., Cheng, L., Prochiantz, A., Mudge, A. W., and Raff, M. C. (2000). Evidence that axon-derived neuregulin promotes oligodendrocyte survival in the developing rat optic nerve. Neuron 28, 81-90.

Fernandez-Borja, M., Janssen, L., Verwoerd, D., Hordijk, P., and Neefjes, J. (2005). RhoB regulates endosome transport by promoting actin assembly on endosomal membranes through Dia1. J Cell Sci 118, 2661-2670.

Fitzner, D., Schneider, A., Kippert, A., Mobius, W., Willig, K. I., Hell, S. W., Bunt, G., Gaus, K., and Simons, M. (2006). Myelin basic protein-dependent plasma membrane reorganization in the formation of myelin. Embo J 25, 5037-5048.

Flores, A. I., Mallon, B. S., Matsui, T., Ogawa, W., Rosenzweig, A., Okamoto, T., and Macklin, W. B. (2000). Akt-mediated survival of oligodendrocytes induced by neuregulins. J Neurosci 20, 7622-7630.

Frost, E., Kiernan, B. W., Faissner, A., and ffrench-Constant, C. (1996). Regulation of oligodendrocyte precursor migration by extracellular matrix: evidence for substrate-specific inhibition of migration by tenascin-C. Dev Neurosci 18, 266-273.

Futter, C. E., Pearse, A., Hewlett, L. J., and Hopkins, C. R. (1996). Multivesicular endosomes containing internalized EGF-EGF receptor complexes mature and then fuse directly with lysosomes. J Cell Biol 132, 1011-1023.

Garrett, W. S., Chen, L. M., Kroschewski, R., Ebersold, M., Turley, S., Trombetta, S., Galan, J. E., and Mellman, I. (2000). Developmental control of endocytosis in dendritic cells by Cdc42. Cell 102, 325-334.

Gasman, S., Kalaidzidis, Y., and Zerial, M. (2003). RhoD regulates endosome dynamics through Diaphanous-related Formin and Src tyrosine kinase. Nat Cell Biol 5, 195-204.

Geminard, C., De Gassart, A., Blanc, L., and Vidal, M. (2004). Degradation of AP2 during reticulocyte maturation enhances binding of hsc70 and Alix to a common site on TFR for sorting into exosomes. Traffic 5, 181-193.

Geuze, H. J., Slot, J. W., Strous, G. J., Lodish, H. F., and Schwartz, A. L. (1983). Intracellular site of asialoglycoprotein receptor-ligand uncoupling: double-label immunoelectron microscopy during receptor-mediated endocytosis. Cell 32, 277-287.

Glebov, O. O., Bright, N. A., and Nichols, B. J. (2006). Flotillin-1 defines a clathrinindependent endocytic pathway in mammalian cells. Nat Cell Biol 8, 46-54.

Griffiths, G., Hoflack, B., Simons, K., Mellman, I., and Kornfeld, S. (1988). The mannose 6phosphate receptor and the biogenesis of lysosomes. Cell 52, 329-341. 
Griffiths, I., Klugmann, M., Anderson, T., Yool, D., Thomson, C., Schwab, M. H., Schneider, A., Zimmermann, F., McCulloch, M., Nadon, N., and Nave, K. A. (1998). Axonal swellings and degeneration in mice lacking the major proteolipid of myelin. Science 280 , 1610-1613.

Gruenberg, J. (2001). The endocytic pathway: a mosaic of domains. Nat Rev Mol Cell Biol 2, 721-730.

Gruenberg, J., Griffiths, G., and Howell, K. E. (1989). Characterization of the early endosome and putative endocytic carrier vesicles in vivo and with an assay of vesicle fusion in vitro. J Cell Biol 108, 1301-1316.

Gruenberg, J., and Stenmark, H. (2004). The biogenesis of multivesicular endosomes. Nat Rev Mol Cell Biol 5, 317-323.

Gulli, M. P., and Peter, M. (2001). Temporal and spatial regulation of Rho-type guaninenucleotide exchange factors: the yeast perspective. Genes Dev 15, 365-379.

Hardy, R., and Reynolds, R. (1991). Proliferation and differentiation potential of rat forebrain oligodendroglial progenitors both in vitro and in vivo. Development 111, 1061-1080.

Hemar, A., Subtil, A., Lieb, M., Morelon, E., Hellio, R., and Dautry-Varsat, A. (1995). Endocytosis of interleukin 2 receptors in human T lymphocytes: distinct intracellular localization and fate of the receptor alpha, beta, and gamma chains. J Cell Biol 129, 55-64.

Henley, J. R., Krueger, E. W., Oswald, B. J., and McNiven, M. A. (1998). Dynaminmediated internalization of caveolae. J Cell Biol 141, 85-99.

Hislop, J. N., Marley, A., and Von Zastrow, M. (2004). Role of mammalian vacuolar protein-sorting proteins in endocytic trafficking of a non-ubiquitinated $\mathrm{G}$ protein-coupled receptor to lysosomes. J Biol Chem 279, 22522-22531.

Hrboticky, N., Feldmeer, T., Essler, M., Wiedemann, A., and Aepfelbacher, M. (2002). Involvement of the GTPase Rho in the cellular uptake of low density lipoprotein by human skin fibroblasts. Biochim Biophys Acta 1580, 123-132.

Hurley, J. H., and Emr, S. D. (2006). The ESCRT complexes: structure and mechanism of a membrane-trafficking network. Annu Rev Biophys Biomol Struct 35, 277-298.

Ishibashi, T., Dakin, K. A., Stevens, B., Lee, P. R., Kozlov, S. V., Stewart, C. L., and Fields, R. D. (2006). Astrocytes promote myelination in response to electrical impulses. Neuron 49, 823-832.

Jensen, A. M., and Raff, M. C. (1997). Continuous observation of multipotential retinal progenitor cells in clonal density culture. Dev Biol 188, 267-279.

Katzmann, D. J., Babst, M., and Emr, S. D. (2001). Ubiquitin-dependent sorting into the multivesicular body pathway requires the function of a conserved endosomal protein sorting complex, ESCRT-I. Cell 106, 145-155.

Katzmann, D. J., Odorizzi, G., and Emr, S. D. (2002). Receptor downregulation and multivesicular-body sorting. Nat Rev Mol Cell Biol 3, 893-905.

Katzmann, D. J., Stefan, C. J., Babst, M., and Emr, S. D. (2003). Vps27 recruits ESCRT machinery to endosomes during MVB sorting. J Cell Biol 162, 413-423. 
Kim, T., Fiedler, K., Madison, D. L., Krueger, W. H., and Pfeiffer, S. E. (1995). Cloning and characterization of MVP17: a developmentally regulated myelin protein in oligodendrocytes. J Neurosci Res 42, 413-422.

Kirkham, M., and Parton, R. G. (2005). Clathrin-independent endocytosis: new insights into caveolae and non-caveolar lipid raft carriers. Biochim Biophys Acta 1746, 349-363.

Kleijmeer, M., Ramm, G., Schuurhuis, D., Griffith, J., Rescigno, M., Ricciardi-Castagnoli, P., Rudensky, A. Y., Ossendorp, F., Melief, C. J., Stoorvogel, W., and Geuze, H. J. (2001). Reorganization of multivesicular bodies regulates MHC class II antigen presentation by dendritic cells. J Cell Biol 155, 53-63.

Klugmann, M., Schwab, M. H., Puhlhofer, A., Schneider, A., Zimmermann, F., Griffiths, I. R., and Nave, K. A. (1997). Assembly of CNS myelin in the absence of proteolipid protein. Neuron 18, 59-70.

Klumperman, J., Kuliawat, R., Griffith, J. M., Geuze, H. J., and Arvan, P. (1998). Mannose 6-phosphate receptors are sorted from immature secretory granules via adaptor protein AP-1, clathrin, and syntaxin 6-positive vesicles. J Cell Biol 141, 359-371.

Kobayashi, T., Stang, E., Fang, K. S., de Moerloose, P., Parton, R. G., and Gruenberg, J. (1998). A lipid associated with the antiphospholipid syndrome regulates endosome structure and function. Nature 392, 193-197.

Kramer, E. M., Koch, T., Niehaus, A., and Trotter, J. (1997). Oligodendrocytes direct glycosyl phosphatidylinositol-anchored proteins to the myelin sheath in glycosphingolipid-rich complexes. J Biol Chem 272, 8937-8945.

Kramer-Albers, E. M., Gehrig-Burger, K., Thiele, C., Trotter, J., and Nave, K. A. (2006). Perturbed interactions of mutant proteolipid protein/DM20 with cholesterol and lipid rafts in oligodendroglia: implications for dysmyelination in spastic paraplegia. J Neurosci 26, 1174311752

Kuliawat, R., Klumperman, J., Ludwig, T., and Arvan, P. (1997). Differential sorting of lysosomal enzymes out of the regulated secretory pathway in pancreatic beta-cells. J Cell Biol 137, 595-608.

Kundra, R., and Kornfeld, S. (1999). Asparagine-linked oligosaccharides protect Lamp-1 and Lamp-2 from intracellular proteolysis. J Biol Chem 274, 31039-31046.

Kurzchalia, T. V., and Parton, R. G. (1999). Membrane microdomains and caveolae. Curr Opin Cell Biol 11, 424-431.

Lakadamyali, M., Rust, M. J., and Zhuang, X. (2006). Ligands for clathrin-mediated endocytosis are differentially sorted into distinct populations of early endosomes. Cell 124, 997-1009.

Lamaze, C., Chuang, T. H., Terlecky, L. J., Bokoch, G. M., and Schmid, S. L. (1996). Regulation of receptor-mediated endocytosis by Rho and Rac. Nature 382, 177-179.

Lamaze, C., Dujeancourt, A., Baba, T., Lo, C. G., Benmerah, A., and Dautry-Varsat, A. (2001). Interleukin 2 receptors and detergent-resistant membrane domains define a clathrinindependent endocytic pathway. Mol Cell 7, 661-671. 
Langford, G. M., Kuznetsov, S. A., Johnson, D., Cohen, D. L., and Weiss, D. G. (1994). Movement of axoplasmic organelles on actin filaments assembled on acrosomal processes: evidence for a barbed-end-directed organelle motor. J Cell Sci 107 ( Pt 8), 2291-2298.

Lappe-Siefke, C., Goebbels, S., Gravel, M., Nicksch, E., Lee, J., Braun, P. E., Griffiths, I. R., and Nave, K. A. (2003). Disruption of Cnp1 uncouples oligodendroglial functions in axonal support and myelination. Nat Genet 33, 366-374.

Le Blanc, I., Luyet, P. P., Pons, V., Ferguson, C., Emans, N., Petiot, A., Mayran, N., Demaurex, N., Faure, J., Sadoul, R., et al. (2005). Endosome-to-cytosol transport of viral nucleocapsids. Nat Cell Biol 7, 653-664.

Levison, S. W., and Goldman, J. E. (1993). Both oligodendrocytes and astrocytes develop from progenitors in the subventricular zone of postnatal rat forebrain. Neuron 10, 201-212.

Liang, X., Draghi, N. A., and Resh, M. D. (2004). Signaling from integrins to Fyn to Rho family GTPases regulates morphologic differentiation of oligodendrocytes. J Neurosci 24, 7140-7149.

Lu, Q., Hope, L. W., Brasch, M., Reinhard, C., and Cohen, S. N. (2003). TSG101 interaction with HRS mediates endosomal trafficking and receptor down-regulation. Proc Natl Acad Sci U S A 100, 7626-7631.

Luhtala, N., and Odorizzi, G. (2004). Bro1 coordinates deubiquitination in the multivesicular body pathway by recruiting Doa4 to endosomes. J Cell Biol 166, 717-729.

Lyubchenko, T. A., Wurth, G. A., and Zweifach, A. (2001). Role of calcium influx in cytotoxic T lymphocyte lytic granule exocytosis during target cell killing. Immunity $15,847-$ 859.

Maldonado-Baez, L., and Wendland, B. (2006). Endocytic adaptors: recruiters, coordinators and regulators. Trends Cell Biol 16, 505-513.

Marks, M. S., and Seabra, M. C. (2001). The melanosome: membrane dynamics in black and white. Nat Rev Mol Cell Biol 2, 738-748.

Marta, C. B., Taylor, C. M., Coetzee, T., Kim, T., Winkler, S., Bansal, R., and Pfeiffer, S. E. (2003). Antibody cross-linking of myelin oligodendrocyte glycoprotein leads to its rapid repartitioning into detergent-insoluble fractions, and altered protein phosphorylation and cell morphology. J Neurosci 23, 5461-5471.

Martinez, I., Chakrabarti, S., Hellevik, T., Morehead, J., Fowler, K., and Andrews, N. W. (2000). Synaptotagmin VII regulates $\mathrm{Ca}(2+)$-dependent exocytosis of lysosomes in fibroblasts. J Cell Biol 148, 1141-1149.

Martin-Serrano, J., Zang, T., and Bieniasz, P. D. (2003). Role of ESCRT-I in retroviral budding. J Virol 77, 4794-4804.

Matsuo, H., Chevallier, J., Mayran, N., Le Blanc, I., Ferguson, C., Faure, J., Blanc, N. S., Matile, S., Dubochet, J., Sadoul, R., et al. (2004). Role of LBPA and Alix in multivesicular liposome formation and endosome organization. Science 303, 531-534.

Maxfield, F. R., and McGraw, T. E. (2004). Endocytic recycling. Nat Rev Mol Cell Biol 5, 121-132. 
Mayran, N., Parton, R. G., and Gruenberg, J. (2003). Annexin II regulates multivesicular endosome biogenesis in the degradation pathway of animal cells. Embo J 22, 3242-3253.

McLaughlin, M., Barrie, J. A., Karim, S., Montague, P., Edgar, J. M., Kirkham, D., Thomson, C. E., and Griffiths, I. R. (2006). Processing of PLP in a model of PelizaeusMerzbacher disease/SPG2 due to the rumpshaker mutation. Glia 53, 715-722.

Meyer, H. H., Wang, Y., and Warren, G. (2002). Direct binding of ubiquitin conjugates by the mammalian p97 adaptor complexes, p47 and Ufd1-Npl4. Embo J 21, 5645-5652.

Mi, S., Miller, R. H., Lee, X., Scott, M. L., Shulag-Morskaya, S., Shao, Z., Chang, J., Thill, G., Levesque, M., Zhang, M., et al. (2005). LINGO-1 negatively regulates myelination by oligodendrocytes. Nat Neurosci 8, 745-751.

Miller, K., Beardmore, J., Kanety, H., Schlessinger, J., and Hopkins, C. R. (1986). Localization of the epidermal growth factor (EGF) receptor within the endosome of EGFstimulated epidermoid carcinoma (A431) cells. J Cell Biol 102, 500-509.

Miller, R. H. (2002). Regulation of oligodendrocyte development in the vertebrate CNS. Prog Neurobiol 67, 451-467.

Mirsky, R., Winter, J., Abney, E. R., Pruss, R. M., Gavrilovic, J., and Raff, M. C. (1980). Myelin-specific proteins and glycolipids in rat Schwann cells and oligodendrocytes in culture. J Cell Biol 84, 483-494.

Mobius, W., van Donselaar, E., Ohno-Iwashita, Y., Shimada, Y., Heijnen, H. F., Slot, J. W., and Geuze, H. J. (2003). Recycling compartments and the internal vesicles of multivesicular bodies harbor most of the cholesterol found in the endocytic pathway. Traffic 4, 222-231.

Monge, M., Kadiiski, D., Jacque, C. M., and Zalc, B. (1986). Oligodendroglial expression and deposition of four major myelin constituents in the myelin sheath during development. An in vivo study. Dev Neurosci 8, 222-235.

Morita, E., and Sundquist, W. I. (2004). Retrovirus budding. Annu Rev Cell Dev Biol 20, 395-425.

Mostov, K. E. (2003). Epithelial polarity and morphogenesis. Methods 30, 189-190.

Mueller, S. C., and Hubbard, A. L. (1986). Receptor-mediated endocytosis of asialoglycoproteins by rat hepatocytes: receptor-positive and receptor-negative endosomes. $\mathrm{J}$ Cell Biol 102, 932-942.

Nishiyama, A., Lin, X. H., Giese, N., Heldin, C. H., and Stallcup, W. B. (1996). Colocalization of NG2 proteoglycan and PDGF alpha-receptor on O2A progenitor cells in the developing rat brain. J Neurosci Res 43, 299-314.

Oh, P., McIntosh, D. P., and Schnitzer, J. E. (1998). Dynamin at the neck of caveolae mediates their budding to form transport vesicles by GTP-driven fission from the plasma membrane of endothelium. J Cell Biol 141, 101-114.

Orlandi, P. A., and Fishman, P. H. (1998). Filipin-dependent inhibition of cholera toxin: evidence for toxin internalization and activation through caveolae-like domains. J Cell Biol 141, 905-915. 
Park, S. K., Solomon, D., and Vartanian, T. (2001). Growth factor control of CNS myelination. Dev Neurosci 23, 327-337.

Parton, R. G., Schrotz, P., Bucci, C., and Gruenberg, J. (1992). Plasticity of early endosomes. J Cell Sci 103 ( Pt 2), 335-348.

Paumet, F., Le Mao, J., Martin, S., Galli, T., David, B., Blank, U., and Roa, M. (2000). Soluble NSF attachment protein receptors (SNAREs) in RBL-2H3 mast cells: functional role of syntaxin 4 in exocytosis and identification of a vesicle-associated membrane protein 8containing secretory compartment. J Immunol 164, 5850-5857.

Pedraza, L., Huang, J. K., and Colman, D. R. (2001). Organizing principles of the axoglial apparatus. Neuron 30, 335-344.

Pelkmans, L. (2005). Secrets of caveolae- and lipid raft-mediated endocytosis revealed by mammalian viruses. Biochim Biophys Acta 1746, 295-304.

Pelkmans, L., Burli, T., Zerial, M., and Helenius, A. (2004). Caveolin-stabilized membrane domains as multifunctional transport and sorting devices in endocytic membrane traffic. Cell 118, 767-780.

Pelkmans, L., Puntener, D., and Helenius, A. (2002). Local actin polymerization and dynamin recruitment in SV40-induced internalization of caveolae. Science 296, 535-539.

Perret, E., Lakkaraju, A., Deborde, S., Schreiner, R., and Rodriguez-Boulan, E. (2005). Evolving endosomes: how many varieties and why? Curr Opin Cell Biol 17, 423-434.

Peters, P. J., Mironov, A., Jr., Peretz, D., van Donselaar, E., Leclerc, E., Erpel, S., DeArmond, S. J., Burton, D. R., Williamson, R. A., Vey, M., and Prusiner, S. B. (2003). Trafficking of prion proteins through a caveolae-mediated endosomal pathway. J Cell Biol 162, 703-717.

Petiot, A., Faure, J., Stenmark, H., and Gruenberg, J. (2003). PI3P signaling regulates receptor sorting but not transport in the endosomal pathway. J Cell Biol 162, 971-979.

Pfeiffer, S. E., Warrington, A. E., and Bansal, R. (1993). The oligodendrocyte and its many cellular processes. Trends Cell Biol 3, 191-197.

Poliak, S., and Peles, E. (2003). The local differentiation of myelinated axons at nodes of Ranvier. Nat Rev Neurosci 4, 968-980.

Pringle, N. P., and Richardson, W. D. (1993). A singularity of PDGF alpha-receptor expression in the dorsoventral axis of the neural tube may define the origin of the oligodendrocyte lineage. Development 117, 525-533.

Puri, V., Watanabe, R., Singh, R. D., Dominguez, M., Brown, J. C., Wheatley, C. L., Marks, D. L., and Pagano, R. E. (2001). Clathrin-dependent and -independent internalization of plasma membrane sphingolipids initiates two Golgi targeting pathways. J Cell Biol 154, 535547.

Radhakrishna, H., Klausner, R. D., and Donaldson, J. G. (1996). Aluminum fluoride stimulates surface protrusions in cells overexpressing the ARF6 GTPase. J Cell Biol 134, 935-947. 
Raiborg, C., Bache, K. G., Gillooly, D. J., Madshus, I. H., Stang, E., and Stenmark, H. (2002). Hrs sorts ubiquitinated proteins into clathrin-coated microdomains of early endosomes. Nat Cell Biol 4, 394-398.

Raiborg, C., Bache, K. G., Mehlum, A., Stang, E., and Stenmark, H. (2001). Hrs recruits clathrin to early endosomes. Embo J 20, 5008-5021.

Raiborg, C., Rusten, T. E., and Stenmark, H. (2003). Protein sorting into multivesicular endosomes. Curr Opin Cell Biol 15, 446-455.

Ridley, A. J. (2006). Rho GTPases and actin dynamics in membrane protrusions and vesicle trafficking. Trends Cell Biol 16, 522-529.

Ridley, A. J., and Hall, A. (1992). The small GTP-binding protein rho regulates the assembly of focal adhesions and actin stress fibers in response to growth factors. Cell 70 , 389-399.

Rink, J., Ghigo, E., Kalaidzidis, Y., and Zerial, M. (2005). Rab conversion as a mechanism of progression from early to late endosomes. Cell 122, 735-749.

Roach, A., Takahashi, N., Pravtcheva, D., Ruddle, F., and Hood, L. (1985). Chromosomal mapping of mouse myelin basic protein gene and structure and transcription of the partially deleted gene in shiverer mutant mice. Cell 42, 149-155.

Russell, M. R., Nickerson, D. P., and Odorizzi, G. (2006). Molecular mechanisms of late endosome morphology, identity and sorting. Curr Opin Cell Biol 18, 422-428.

Sabharanjak, S., Sharma, P., Parton, R. G., and Mayor, S. (2002). GPI-anchored proteins are delivered to recycling endosomes via a distinct cdc42-regulated, clathrin-independent pinocytic pathway. Dev Cell 2, 411-423.

Salzer, J. L. (2003). Polarized domains of myelinated axons. Neuron 40, 297-318.

Sandilands, E., Cans, C., Fincham, V. J., Brunton, V. G., Mellor, H., Prendergast, G. C., Norman, J. C., Superti-Furga, G., and Frame, M. C. (2004). RhoB and actin polymerization coordinate Src activation with endosome-mediated delivery to the membrane. Dev Cell 7 , 855-869.

Sauvonnet, N., Dujeancourt, A., and Dautry-Varsat, A. (2005). Cortactin and dynamin are required for the clathrin-independent endocytosis of gammac cytokine receptor. $\mathrm{J}$ Cell Biol 168, 155-163.

Scherer, S. S., and Arroyo, E. J. (2002). Recent progress on the molecular organization of myelinated axons. J Peripher Nerv Syst 7, 1-12.

Schneider, A., Lander, H., Schulz, G., Wolburg, H., Nave, K. A., Schulz, J. B., and Simons, M. (2005). Palmitoylation is a sorting determinant for transport to the myelin membrane. J Cell Sci 118, 2415-2423.

Shiota, C., Miura, M., and Mikoshiba, K. (1989). Developmental profile and differential localization of mRNAs of myelin proteins (MBP and PLP) in oligodendrocytes in the brain and in culture. Brain Res Dev Brain Res 45, 83-94. 
Sigismund, S., Woelk, T., Puri, C., Maspero, E., Tacchetti, C., Transidico, P., Di Fiore, P. P., and Polo, S. (2005). Clathrin-independent endocytosis of ubiquitinated cargos. Proc Natl Acad Sci U S A 102, 2760-2765.

Simons, M., Kramer, E. M., Macchi, P., Rathke-Hartlieb, S., Trotter, J., Nave, K. A., and Schulz, J. B. (2002). Overexpression of the myelin proteolipid protein leads to accumulation of cholesterol and proteolipid protein in endosomes/lysosomes: implications for PelizaeusMerzbacher disease. J Cell Biol 157, 327-336.

Simons, M., Kramer, E. M., Thiele, C., Stoffel, W., and Trotter, J. (2000). Assembly of myelin by association of proteolipid protein with cholesterol- and galactosylceramide-rich membrane domains. J Cell Biol 151, 143-154.

Simons, M., and Trajkovic, K. (2006) Neuron-glia communication in the control of oligodendrocyte function and myelin biogenesis. J Cell Sci 119, 4381-9.

Slagsvold, T., Pattni, K., Malerod, L., and Stenmark, H. (2006). Endosomal and nonendosomal functions of ESCRT proteins. Trends Cell Biol 16, 317-326.

Sobo, K., Chevallier, J., Parton, R. G., Gruenberg, J., and van der Goot, F. G. (2007). Diversity of raft-like domains in late endosomes. PLoS ONE 2, e391.

Solly, S. K., Thomas, J. L., Monge, M., Demerens, C., Lubetzki, C., Gardinier, M. V., Matthieu, J. M., and Zalc, B. (1996). Myelin/oligodendrocyte glycoprotein (MOG) expression is associated with myelin deposition. Glia 18, 39-48.

Stenmark, H., Parton, R. G., Steele-Mortimer, O., Lutcke, A., Gruenberg, J., and Zerial, M. (1994). Inhibition of rab5 GTPase activity stimulates membrane fusion in endocytosis. Embo J 13, 1287-1296.

Stevens, B., and Fields, R. D. (2000). Response of Schwann cells to action potentials in development. Science 287, 2267-2271.

Stevens, B., Porta, S., Haak, L. L., Gallo, V., and Fields, R. D. (2002). Adenosine: a neuron-glial transmitter promoting myelination in the CNS in response to action potentials. Neuron 36, 855-868.

Stoorvogel, W., Kleijmeer, M. J., Geuze, H. J., and Raposo, G. (2002). The biogenesis and functions of exosomes. Traffic 3, 321-330.

Strack, B., Calistri, A., Craig, S., Popova, E., and Gottlinger, H. G. (2003). AIP1/ALIX is a binding partner for HIV-1 p6 and EIAV p9 functioning in virus budding. Cell 114, 689-699.

Sunyach, C., Jen, A., Deng, J., Fitzgerald, K. T., Frobert, Y., Grassi, J., McCaffrey, M. W., and Morris, R. (2003). The mechanism of internalization of glycosylphosphatidylinositolanchored prion protein. Embo J 22, 3591-3601.

Symons, M., and Rusk, N. (2003). Control of vesicular trafficking by Rho GTPases. Curr Biol 13, R409-418.

Symons, M., and Settleman, J. (2000). Rho family GTPases: more than simple switches. Trends Cell Biol 10, 415-419. 
Tagawa, A., Mezzacasa, A., Hayer, A., Longatti, A., Pelkmans, L., and Helenius, A. (2005). Assembly and trafficking of caveolar domains in the cell: caveolae as stable, cargotriggered, vesicular transporters. J Cell Biol 170, 769-779.

Taylor, C. M., Coetzee, T., and Pfeiffer, S. E. (2002). Detergent-insoluble glycosphingolipid/cholesterol microdomains of the myelin membrane. J Neurochem 81, 9931004.

Temple, S., and Raff, M. C. (1986). Clonal analysis of oligodendrocyte development in culture: evidence for a developmental clock that counts cell divisions. Cell 44, 773-779.

Theos, A. C., Truschel, S. T., Tenza, D., Hurbain, I., Harper, D. C., Berson, J. F., Thomas, P. C., Raposo, G., and Marks, M. S. (2006). A lumenal domain-dependent pathway for sorting to intralumenal vesicles of multivesicular endosomes involved in organelle morphogenesis.

Dev Cell 10, 343-354.

Timsit, S. G., Bally-Cuif, L., Colman, D. R., and Zalc, B. (1992). DM-20 mRNA is expressed during the embryonic development of the nervous system of the mouse. $\mathrm{J}$ Neurochem 58, 1172-1175.

Trombetta, E. S., Ebersold, M., Garrett, W., Pypaert, M., and Mellman, I. (2003). Activation of lysosomal function during dendritic cell maturation. Science 299, 1400-1403.

Trombetta, E. S., and Mellman, I. (2005). Cell biology of antigen processing in vitro and in vivo. Annu Rev Immunol 23, 975-1028.

Urbe, S., Sachse, M., Row, P. E., Preisinger, C., Barr, F. A., Strous, G., Klumperman, J., and Clague, M. J. (2003). The UIM domain of Hrs couples receptor sorting to vesicle formation. J Cell Sci 116, 4169-4179.

van der Goot, F. G., and Gruenberg, J. (2006). Intra-endosomal membrane traffic. Trends Cell Biol 16, 514-521.

Vogler, O., Krummenerl, P., Schmidt, M., Jakobs, K. H., and Van Koppen, C. J. (1999). RhoA-sensitive trafficking of muscarinic acetylcholine receptors. J Pharmacol Exp Ther 288, 36-42.

von Schwedler, U. K., Stuchell, M., Muller, B., Ward, D. M., Chung, H. Y., Morita, E., Wang, H. E., Davis, T., He, G. P., Cimbora, D. M., et al. (2003). The protein network of HIV budding. Cell 114, 701-713.

Vonderheit, A., and Helenius, A. (2005). Rab7 associates with early endosomes to mediate sorting and transport of Semliki forest virus to late endosomes. PLoS Biol 3, e233.

Wang, S., Sdrulla, A. D., diSibio, G., Bush, G., Nofziger, D., Hicks, C., Weinmaster, G., and Barres, B. A. (1998). Notch receptor activation inhibits oligodendrocyte differentiation. Neuron 21, 63-75.

Weimbs, T., and Stoffel, W. (1992). Proteolipid protein (PLP) of CNS myelin: positions of free, disulfide-bonded, and fatty acid thioester-linked cysteine residues and implications for the membrane topology of PLP. Biochemistry 31, 12289-12296. 
White, I. J., Bailey, L. M., Aghakhani, M. R., Moss, S. E., and Futter, C. E. (2006). EGF stimulates annexin 1-dependent inward vesiculation in a multivesicular endosome subpopulation. Embo J 25, 1-12.

Wight, P.A., Duchala, C.S., Readhead, C., and Macklin, W.B. (1993). A myelin proteolipid protein-LacZ fusion protein is developmentally regulated and targeted to the myelin membrane in transgenic mice. JCB 123, 443-454.

Yiu, G., and He, Z. (2006). Glial inhibition of CNS axon regeneration. Nat Rev Neurosci 7, 617-627.

Yu, W. P., Collarini, E. J., Pringle, N. P., and Richardson, W. D. (1994). Embryonic expression of myelin genes: evidence for a focal source of oligodendrocyte precursors in the ventricular zone of the neural tube. Neuron 12, 1353-1362. 


\section{Acknowledgements}

This thesis has been done at the Center of Biochemistry and Molecular Cell Biology, University of Göttingen, and at the Max Planck Institute for experimental Medicine in Göttingen.

I would like to thank, first and foremost, my advisor, PD Dr. Mikael Simons for the excellent guidance, great working conditions, encouragement and support throughout my career.

I am grateful to the members of my committee, Prof. Dr. Ernst Wimmer and Prof. Dr. Detlef Doenecke for their interest in this research.

Special thanks to Angelika Kippert and Chieh Hsu for the nice and fruitful collaboration and contribution in publications.

I am also grateful to the members of my group for their cordial help over the years spent together in our laboratory and for creating a nice working atmosphere.

Many thanks to Ana Pekanovic, for bringing me to this group, sharing her experience with me and providing helpful instructions in experimental practice.

I am thankful to my friends and colleagues who made my time in Germany unforgettable.

Finally, I am deeply indebted to my family and to Dirk Fitzner for their endless support and understanding. 


\section{CURRICULUM VITAE}

Vorname:

Nachname:

Geburtsdatum:

Geburtsort:

Nationalität:

1984-1992

1992-1996

1996-2001

2001-2003

2003-2007

2003-2004

2004-2007
Katarina

Trajkovic

28.11.1977

Pozarevac/Serbien

Serbisch

Grundschule in Pozarevac, Serbien

Gymnasium in Pozarevac, Serbien

Universität Belgrad, Fakultät für Biologie, Serbien, Diplom in Molekularbiologie und Physiologie, Titel der Diplomarbeit:

Beziehung zwischen Allelen des COLIA1Gens und der Knochendichte in einer Population jugoslawischer Frauen.

Masterstudiengang, Fakultät für Biologie, Studiengruppe Neurobiologie, Universität Belgrad, nicht abgeschlossen

Dissertationsarbeit unter Anleitung von Dr. Mikael Simons

Klinik für Neurologie, Universität Tübingen, Deutschland

Zentrum für Biochemie und molekulare Zellbiologie, Universität Göttingen, MPI für Experimentelle Medizin, Göttingen

Publikationsliste:

1. Schneider, A., Araujo, G.W., Trajkovic, K., Herrmann, M.M., Merkler, D., Mandelkov, E.M., Weissert, R., Simons, M. (2004). Hyperphosphorylation and aggregation of tau in experimental autoimmune encephalomyelitis. J Biol Chem 279, 55833-9. 
2. Trajkovic, K., Dhaunchak, A.S., Goncalves, J.T., Wenzel, D., Schneider, A., Bunt, G., Nave, K.A., Simons, M. (2006). Neuron to glia signaling triggers myelin membrane exocytosis from endosomal storage sites. J Cell Biol 172, 937-48.

3. Simons, M., and Trajkovic, K. (2006) Neuron-glia communication in the control of oligodendrocyte function and myelin biogenesis. J Cell Sci 119, 4381-9.

4. Kippert, A. ", Trajkovic, K. *, Rajendran, L., Ries, J., Simons, M. (2006). Rho regulates membrane transport in the endocytic pathway to control plasma membrane specialization in oligodendroglial cells. J Neurosci 27, 3560-70. * equal contribution to the first authorship

5. Trajkovic, K., Hsu, C., Wenzel, D., Brugger, B., Simons, M. (manuscript in preparation). A novel pathway in the formation of intraendosomal membrane and exosome biogenesis.

Göttingen, den 25.05.2007 